\title{
Unified Charge Vectors (UCV)
}

\section{Theory}

\author{
By Noam Why \\ E-mail: NoamWhy@Gmail.com \\ Website: NoamWhy.com \\ Papers: Github.com/NoamWhy
}

January $5^{\text {th }} 2021$

\begin{abstract}
A grand unification of electroweak and strong forces is presented based on a new idea called Unified Charged Vectors. Using this new concept, it is shown that:
\end{abstract}

1. The electroweak and strong forces can be unified into a single electro-weak-strong force.

2. The various charges and coupling constants of fermions (like the electroweak mixing angle) can be predicted, rather than presupposed.

3. The Lagrangian symmetries can be predicted rather than presupposed.

4. The standard model Lagrangian can be cast into a simple unified form. 


\section{Unified Charge Vectors Theory}

Table of Contents

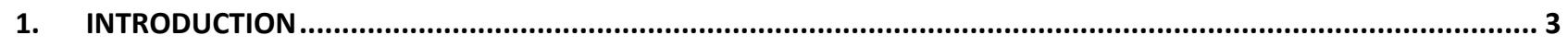

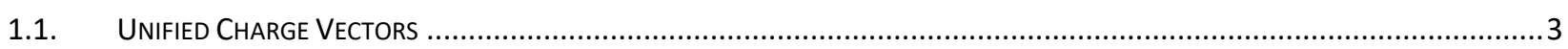

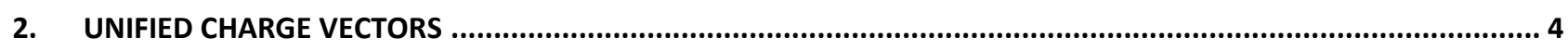

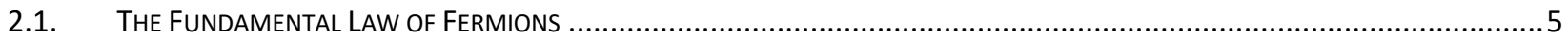

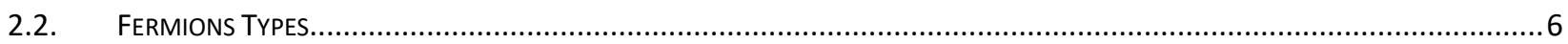

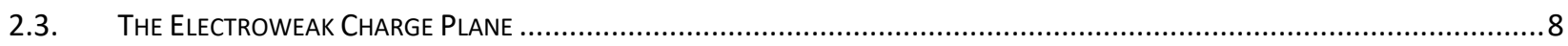

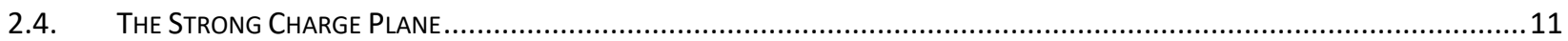

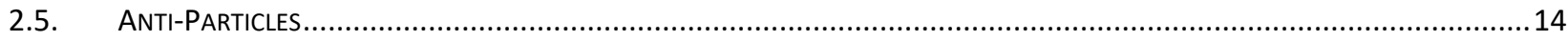

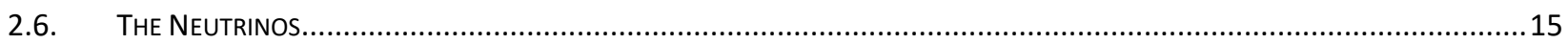

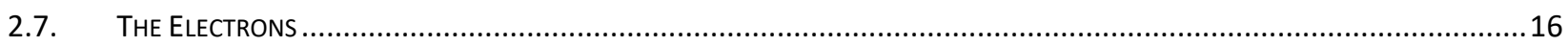

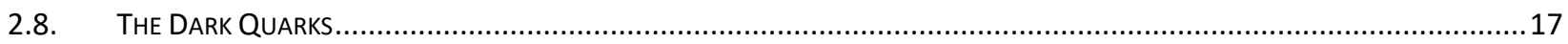

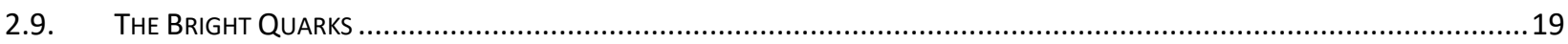

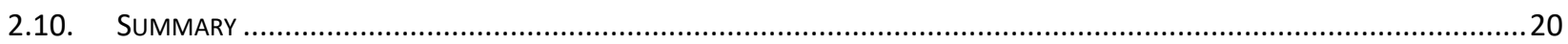

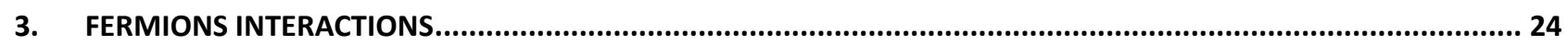

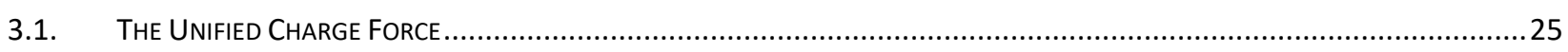

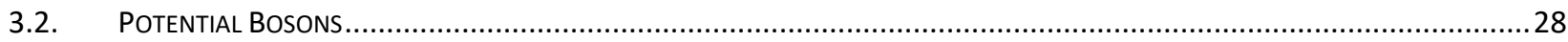

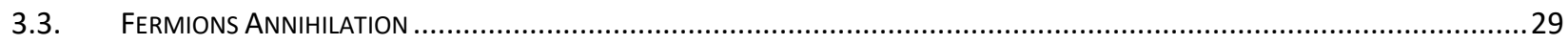

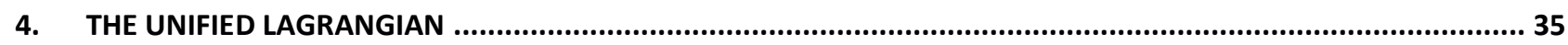

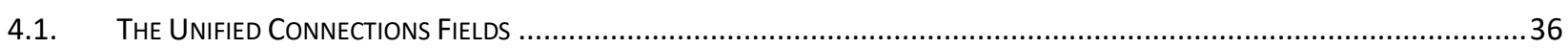

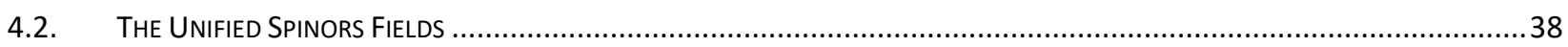

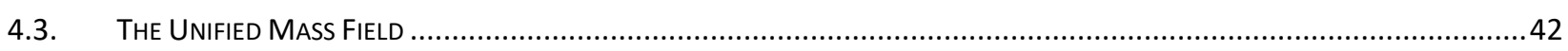

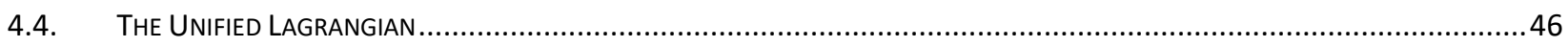

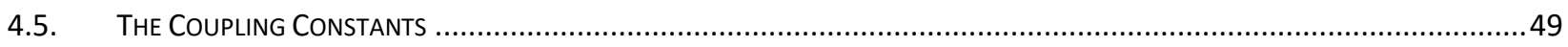

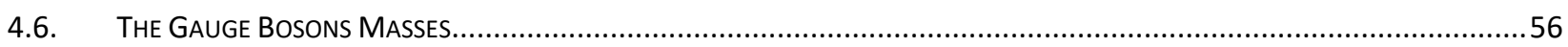

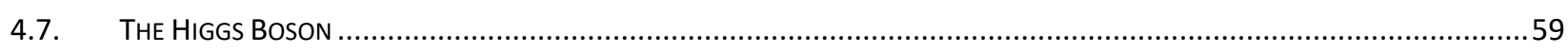

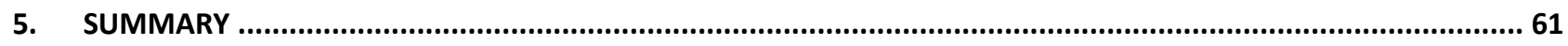

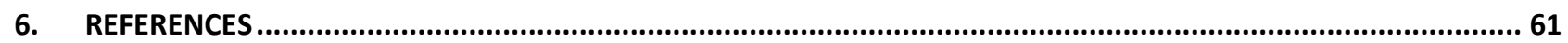




\section{Unified Charge Vectors Theory}

\section{Introduction}

\subsection{Unified Charge Vectors}

To understand the basic idea behind UCV theory, let us begin by considering two electrically charged particles with charges $q^{a}$ and $q^{b}$, located at a distance $r$ from each other. The electric potential energy between these two particles is given by the following familiar equation:

$$
\mathrm{E}=\frac{\alpha}{r} q^{a} q^{b} \quad \text { where } \quad \alpha \stackrel{\text { def }}{=} \frac{\mathrm{e}^{2}}{4 \pi \varepsilon_{0}} \text { and } \hbar=c=1
$$

UCV theory is based on the idea of promoting the charges $q^{a}$ and $q^{b}$ into four-dimensional vectors, denoted by $\overleftrightarrow{\mathbf{q}}^{a}$ and $\overleftrightarrow{\mathbf{q}}^{b}$, and referred to as Unified Charge Vectors. It is essential to realize that these are not spacetime four-vectors but rather vectors in an abstract four-dimensional Charge Space. This implies that charge vectors are not affected by spacetime transformations of any sort (e.g., rotations).

Furthermore, the notation $\overleftrightarrow{\mathbf{q}}$ actually stands for two different charge vectors for each particle, namely $\overleftarrow{\mathrm{q}}$ and $\overrightarrow{\mathbf{q}}$. We refer to these two charge vectors respectively as the Left \& Right Charge Vectors of a particle.

With the help of the above charge vectors associated with each of the particles, we postulate that the total nongravitational (i.e., electro-weak-strong) classical potential energy between any two particles is given by:

$$
E=\frac{\alpha}{r} \frac{1}{2}\left(\overleftarrow{\mathrm{q}}^{a} \cdot \overleftarrow{\mathrm{q}}^{b}+\overrightarrow{\mathbf{q}}^{a} \cdot \overrightarrow{\mathbf{q}}^{b}\right)
$$

(Strictly speaking, equation (1.1-2) cannot possibly describe the weak potential correctly for the simple reason that the weak potential decays exponentially. However, this turns out to be a minor issue that we shall easily fix later on in (3.1-6), and so for the purpose of this introduction, it can safely be ignored.)

Equation (1.1-2) expresses the interaction between any two particles as a function of the dot product of their corresponding left and right unified charge vectors. This suggests the appealing idea that the only difference between the various fermions is their mass and their charge vector orientation.

In particular, every orientation of the charge vectors defines a new type of fermion, and so theoretically, there should exist infinitely many kinds of fermions. Since this stands in contradiction to observations, we further postulate that the unified charge vectors have to satisfy some Charge Quantization Law that will restrict the possible values they can obtain. As it turns out, such a charge quantization law can neatly be formulated as follows:

$$
\overleftrightarrow{\mathbf{q}}=\hat{\mathbf{Z}}\left(\frac{\widehat{\mathbf{z}}_{\theta} \cdot \boldsymbol{s}}{\sqrt{3} \theta_{\mathrm{c}}}+\frac{s_{0}-\overleftrightarrow{\Pi} s}{4 \theta_{c} \theta_{\mathrm{s}}}\right)-\frac{\boldsymbol{s}}{\sqrt{3}}
$$

The specific details of this equation are the subject of the next chapter. As we shall see, it encapsulates the standard model of elementary particles in a fantastically compact form. 


\section{Unified Charge Vectors Theory}

\section{Unified Charge Vectors}

In this chapter, we present the fermions charge quantization law and use it to derive the various fermions types.

We then proceed to develop the concepts of the electroweak and strong charge planes.

Finally, we compute the charge vectors of each of the fermions types. 


\section{Unified Charge Vectors Theory}

\subsection{The Fundamental Law of Fermions}

UCV theory is based on the following Fundamental Law of Fermions, according to which:

Every fermion is characterized by two four-dimensional Unified Charge Vectors, $\overleftarrow{\mathrm{q}}$ and $\overrightarrow{\mathbf{q}}$, satisfying the following Fermions Charge Quantization Law:

$$
\overleftrightarrow{\mathbf{q}}=\hat{\mathbf{z}}\left(\frac{\widehat{\mathbf{Z}}_{\theta} \cdot s}{\sqrt{3} \theta_{c}}+\frac{s_{0}-\overleftrightarrow{\Pi} s}{4 \theta_{c} \theta_{s}}\right)-\frac{s}{\sqrt{3}}
$$

Where:

- The upper arrows in (2.1-1) do not represent vector notation but instead denote a distinction between left and right entities. Hence, we write:

$$
\overleftarrow{\mathbf{q}} \stackrel{\text { def }}{=}\left[\overleftarrow{q}_{0}, \overleftarrow{q}_{1}, \overleftarrow{q}_{2}, \overleftarrow{q}_{3}\right] \text { and } \overrightarrow{\mathbf{q}} \stackrel{\text { def }}{=}\left[\vec{q}_{0}, \vec{q}_{1}, \vec{q}_{2}, \vec{q}_{3}\right] \text { or collectively } \stackrel{\leftrightarrow \mathbf{q}}{\stackrel{\text { def }}{=}}\left[\overleftrightarrow{q}_{0}, \overleftrightarrow{q}_{1}, \overleftrightarrow{q}_{2}, \overleftrightarrow{q}_{3}\right]
$$

- The vector $\boldsymbol{s}$ is referred to as the Signature of $\overleftrightarrow{\mathbf{q}}$, and each of its elements can independently obtain either the value +1 or -1 . Hence, we have:

$$
\boldsymbol{s} \stackrel{\text { def }}{=}\left[s_{0}, s_{1}, s_{2}, s_{3}\right]=[ \pm 1, \pm 1, \pm 1, \pm 1]
$$

We also define the left and right Total Signature Products as follows:

$$
\overleftarrow{\Pi}_{s} \stackrel{\text { def }}{=}-s_{0} s_{1} s_{2} s_{3} \text { and } \vec{\Pi} s \stackrel{\text { def }}{=} s_{0} s_{1} s_{2} s_{3}
$$

- The vectors $\hat{\mathbf{Z}}$ and $\hat{\mathbf{Z}}_{\theta}$ are defined as follows:

$$
\begin{gathered}
\widehat{\mathbf{Z}}_{\theta} \stackrel{\text { def }}{=} \theta_{\mathrm{c}} \widehat{\mathbf{Z}}+\theta_{\mathrm{s}} \widehat{\mathbf{A}} ; \hat{\mathbf{Z}} \stackrel{\text { def }}{=} \frac{1}{2}[1,1,1,1] ; \widehat{\mathbf{A}} \stackrel{\text { def }}{=} \frac{1}{\sqrt{12}}[-3,1,1,1] \\
\theta_{S} \stackrel{\text { def }}{=} \sin (\theta) ; \theta_{c} \stackrel{\text { def }}{=} \cos (\theta)
\end{gathered}
$$

Where $\theta$ is a constant called the Electroweak Mixing Angle and is computed later on in (4.5-34). 


\section{Unified Charge Vectors Theory}

\subsection{Fermions Types}

Equation (2.1-1) implies that the two charge vectors $\overleftrightarrow{\mathbf{q}}$ can be fully determined by the signature $\boldsymbol{s}$, defined in (2.1-3).

Since $s$ is composed out of four components, each of which can independently obtain only the value +1 or -1 , we conclude that there exist exactly $2^{4}$ (i.e., 16) different signatures, and hence at most 16 different charge vectors.

Fermions that share the same signature (and hence the same charge vectors) are said to be of the same type. Such fermions can only differ in their mass (e.g., electrons and muons) and are said to belong to a different Generation within that same type. Hence, by definition, all generations of a given type share precisely the same charge vectors and differ in their mass only.

The following table lists all the possible fermions types and provides a name, a symbol, and a coloring mnemonic to each. It is essential to emphasize that the term "Dark" below has absolutely nothing to do with the notion of dark-matter discussed in cosmology.

\begin{tabular}{|c|c|c|c|c|c|c|}
\hline$\#$ & Fermions Type & Symbol & Signature & Fermions Type & Symbol & Signature \\
\hline 1 & Dark Neutrinos & $\overleftrightarrow{\mathbf{q}}^{v}$ & ++++ & Bright Anti-Neutrinos & $\overleftrightarrow{\mathbf{q}}^{v}$ & ---- \\
\hline 2 & Dark Red Quarks & $\overleftrightarrow{\mathbf{q}}^{\mathrm{r}}$ & ++-- & Bright Anti Red Quarks & $\overleftrightarrow{\mathbf{q}}^{\mathrm{r}}$ & --++ \\
\hline 3 & Dark Green Quarks & $\overleftrightarrow{\mathbf{q}}^{\mathrm{g}}$ & +-+- & Bright Anti Green Quarks & $\overleftrightarrow{\mathbf{q}}^{\mathrm{g}}$ & -+-+ \\
\hline 4 & Dark Blue Quarks & $\overleftrightarrow{\mathbf{q}}^{\mathrm{b}}$ & +--+ & Bright Anti Blue Quarks & ${\overleftrightarrow{\mathbf{q}^{\mathrm{q}}}}^{\mathrm{b}}$ & -++- \\
\hline 5 & Bright El & $\stackrel{\leftrightarrow}{\mathrm{g}}^{e}$ & & Dark Anti-Electrons & $\stackrel{\leftrightarrow}{\Omega} e$ & \\
\hline 6 & Bright Red Quarks & $\overleftrightarrow{\mathrm{q}}^{\mathrm{R}}$ & -+-- & Dark Anti Red Quarks & $\stackrel{\leftrightarrow}{\leftrightarrows} \mathrm{R}$ & +-++ \\
\hline 7 & Bright Green Quarks & $\stackrel{\leftrightarrow}{\mathrm{q}}^{\mathrm{G}}$ & --+- & Dark Anti Green Quarks & $\stackrel{\leftrightarrow}{\mathrm{g}} G$ & ++-+ \\
\hline 8 & Bright Blue Quarks & $\overleftrightarrow{\mathrm{q}}^{\mathrm{B}}$ & ---+ & Dark Anti Blue Quarks & $\stackrel{\leftrightarrow}{\mathrm{q}}^{\mathrm{B}}$ & +++- \\
\hline
\end{tabular}

\section{Fermions types}

Table (2.2-1) has on its left side all the so-called Positive Signatures (defined by having $s_{1} s_{2} s_{3}=+1$ ) and on its right side all the Negative Signatures, or Anti-Signatures (defined by having $s_{1} s_{2} s_{3}=-1$ ). We shall say that two fermions are of the same Breed if they have the same or the opposite signature.

In addition to that, the table assigns to $s_{1}, s_{2}$, and $s_{3}$, respectively, the colors Red, Green, and Blue. This allows us to refer to Neutrinos and Electrons as Colorless since they contain a uniform mix of red, green, and blue.

On the other hand, quarks are Colored and are arranged in four sets of three types each. As we shall see, Dark Quarks correspond to the up/charm/top quarks type, while the Bright Quarks correspond to the down/strange/bottom quarks type. 


\section{Unified Charge Vectors Theory}

The terms Dark \& Bright refer to the sign of $s_{0}$, where $s_{0}=+1$ is referred to as Dark, and $s_{0}=-1$ as Bright. The metaphor here is that $s_{0}$ acts as a "Dark Mask", so its sign can turn a fermion brighter or darker. This is why the anti of a dark signature is bright, and vice versa.

It is easy to see that every bright type has a corresponding dark type obtained by flipping the sign of $s_{0}$. The following table lists this bright-dark correspondence:

\begin{tabular}{|c|c|}
\hline \multicolumn{2}{|c|}{ Bright $\rightarrow$ Dark } \\
\hline$\overleftrightarrow{\mathrm{q}}^{e} \rightarrow \overleftrightarrow{\mathbf{q}}^{v}$ & $\overleftrightarrow{\leftrightarrow}^{v} \rightarrow \overleftrightarrow{\mathbf{q}}^{e}$ \\
\hline$\overleftrightarrow{\mathrm{q}}^{\mathrm{R}} \rightarrow \overleftrightarrow{\mathbf{q}}^{\mathrm{r}}$ & $\overleftrightarrow{\mathrm{q}}^{\mathrm{r}} \rightarrow \overleftrightarrow{\mathbf{q}}^{\mathrm{R}}$ \\
\hline$\overleftrightarrow{\mathrm{q}}^{\mathrm{G}} \rightarrow \overleftrightarrow{\mathbf{q}}^{\mathrm{g}}$ & $\stackrel{\leftrightarrow}{\mathrm{q}}^{\mathrm{g}} \rightarrow \stackrel{\leftrightarrow}{\mathbf{q}}^{\mathrm{G}}$ \\
\hline$\stackrel{\leftrightarrow}{\mathrm{q}}^{\mathrm{B}} \rightarrow \overleftrightarrow{\mathbf{q}}^{\mathrm{b}}$ & $\stackrel{\leftrightarrow}{\mathrm{q}}^{\mathrm{b}} \rightarrow \stackrel{\overleftrightarrow{\mathbf{q}}}{\mathrm{B}}^{\mathrm{B}}$ \\
\hline
\end{tabular}

Bright-Dark Correspondence

As we shall see, Bright-Dark correspondence lies at the heart of what is known as Weak-Interactions. For example, the so-called Beta-Decay, which consists of a down-quark turning into an up-quark while emitting a $\mathbf{W}^{-}$, is nothing but the process of Dimming a quark, i.e., turning it from bright to dark, where the extra brightness is carried away by the $\mathbf{W}^{-}$which later decays into a bright electron and a bright anti-neutrino.

This leads to the realization that weak interactions are better described as Brightness-Interactions since they change fermions' brightness. Likewise, beta-decay is better described as Quark-Dimming.

We shall develop the full theory of fermions interactions in the next chapter. 


\section{Unified Charge Vectors Theory}

\subsection{The Electroweak Charge Plane}

The charge vectors $\overleftrightarrow{\mathbf{q}}$ can be written as the following linear combination:

$$
\overleftrightarrow{\mathbf{q}}=\sum_{\alpha=0}^{3} \widehat{\mathbf{D}}_{\alpha} \overleftrightarrow{q}_{\alpha} \stackrel{\text { def }}{=} \widehat{\mathbf{D}}_{0} \overleftrightarrow{q}_{0}+\widehat{\mathbf{D}}_{1} \overleftrightarrow{q}_{1}+\widehat{\mathbf{D}}_{2} \overleftrightarrow{q}_{2}+\widehat{\mathbf{D}}_{3} \overleftrightarrow{q}_{3}
$$

Where:

$$
\widehat{\mathbf{D}}_{0} \stackrel{\text { def }}{=}[1,0,0,0] ; \quad \widehat{\mathbf{D}}_{1} \stackrel{\text { def }}{=}[0,1,0,0] ; \quad \widehat{\mathbf{D}}_{2} \stackrel{\text { def }}{=}[0,0,1,0] ; \quad \widehat{\mathbf{D}}_{3} \stackrel{\text { def }}{=}[0,0,0,1]
$$

If we refer to the four vectors $\widehat{\mathbf{D}}_{0}, \widehat{\mathbf{D}}_{1}, \widehat{\mathbf{D}}_{2}, \widehat{\mathbf{D}}_{3}$ as Charge Directions, then (2.3-1) simply expresses the fact that $\overleftrightarrow{q}_{n}$ is just the length of the component of $\overleftrightarrow{\mathbf{q}}$ along the $\widehat{\mathbf{D}}_{n}$ charge direction.

It is easy to see that $\widehat{\mathbf{D}}_{0}, \widehat{\mathbf{D}}_{1}, \widehat{\mathbf{D}}_{2}, \widehat{\mathbf{D}}_{3}$ are orthonormal to each other, i.e.,

So:

$$
\widehat{\mathbf{D}}_{n} \cdot \widehat{\mathbf{D}}_{m}=\boldsymbol{\delta}_{n m} \stackrel{\text { def }}{=} \begin{cases}1 & \text { when } n=m \\ 0 & \text { when } n \neq m\end{cases}
$$

$$
\widehat{\mathbf{D}}_{n} \cdot \overleftrightarrow{\mathbf{q}}=\sum_{\alpha=0}^{3} \widehat{\mathbf{D}}_{n} \cdot\left(\widehat{\mathbf{D}}_{\alpha} \overleftrightarrow{q}_{\alpha}\right)=\overleftrightarrow{q}_{n}
$$

Hence, $\widehat{\mathbf{D}}_{0}, \widehat{\mathbf{D}}_{1}, \widehat{\mathbf{D}}_{2}, \widehat{\mathbf{D}}_{3}$ can serve as a basis for the linear space of all charge vectors. Surely there is nothing special about this particular basis. Indeed, we may choose any other orthonormal basis $\widehat{\mathbf{X}}_{0}, \widehat{\mathbf{X}}_{1}, \widehat{\mathbf{X}}_{2}, \widehat{\mathbf{X}}_{3}$, and have:

$$
\overleftrightarrow{\mathbf{q}}=\sum_{\alpha=0}^{3} \widehat{\mathbf{X}}_{\alpha} \stackrel{\leftrightarrow}{q}_{X_{\alpha}} \quad \text { where } \stackrel{\leftrightarrow}{\mathrm{q}}_{X_{n}} \stackrel{\text { def }}{=} \widehat{\mathbf{X}}_{n} \cdot \overleftrightarrow{\mathbf{q}}
$$

We can then refer to $\overleftrightarrow{\mathrm{q}}_{X_{n}}$ as the charge component along the $\widehat{\mathbf{X}}_{n}$ charge direction.

Having realized that, we take a second look at equation (2.1-1) and notice that it is making use of the charge directions defined in (2.1-5), i.e.,

$$
\begin{gathered}
\widehat{\mathbf{Z}} \stackrel{\text { def }}{=} \frac{1}{2}[1,1,1,1] ; \quad \widehat{\mathbf{A}} \stackrel{\text { def }}{=} \frac{1}{\sqrt{12}}[-3,1,1,1] ; \quad \hat{\mathbf{Z}}_{\theta} \stackrel{\text { def }}{=} \theta_{\mathbf{c}} \widehat{\mathbf{Z}}+\theta_{\mathbf{S}} \widehat{\mathbf{A}} \\
\theta_{S} \stackrel{\text { def }}{=} \sin (\theta) ; \theta_{c} \stackrel{\text { def }}{=} \cos (\theta)
\end{gathered}
$$

A quick computation shows us that $\widehat{\mathbf{Z}}$ and $\widehat{\mathbf{A}}$ are orthonormal to each other, i.e.,

$$
\widehat{\mathbf{Z}} \cdot \widehat{\mathbf{Z}}=\widehat{\mathbf{A}} \cdot \widehat{\mathbf{A}}=1 \quad \text { and } \quad \widehat{\mathbf{Z}} \cdot \widehat{\mathbf{A}}=0
$$




\section{Unified Charge Vectors Theory}

The relations between the three vectors $\widehat{\mathbf{Z}}_{\theta}, \widehat{\mathbf{Z}}$, and $\widehat{\mathbf{A}}$, are best explained by the following diagram:

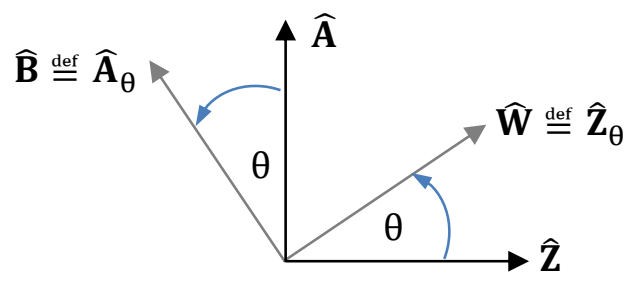

The relations between $\widehat{\boldsymbol{Z}}, \widehat{\boldsymbol{A}}$ and $\widehat{\boldsymbol{Z}}_{\theta}, \widehat{\boldsymbol{A}}_{\theta}$

As we can see, $\widehat{\mathbf{Z}}_{\theta}$ is simply the rotation of the vector $\widehat{\mathbf{Z}}$ by an angle $\theta$ in the plane spanned by $\widehat{\mathbf{Z}}$ and $\widehat{\mathbf{A}}$. This suggests the use of another vector, $\widehat{\mathbf{A}}_{\theta}$, which is obtained by rotating $\widehat{\mathbf{A}}$ by the same angle $\theta$. We therefore have:

$$
\left[\begin{array}{c}
\widehat{\mathbf{B}} \\
\widehat{\mathbf{W}}
\end{array}\right] \stackrel{\text { def }}{=}\left[\begin{array}{l}
\widehat{\mathbf{A}}_{\theta} \\
\widehat{\mathbf{Z}}_{\theta}
\end{array}\right] \stackrel{\text { def }}{=}\left[\begin{array}{rr}
\theta_{\mathrm{c}} & -\theta_{s} \\
\theta_{s} & \theta_{\mathrm{c}}
\end{array}\right]\left[\begin{array}{l}
\widehat{\mathbf{A}} \\
\widehat{\mathbf{Z}}
\end{array}\right]=\left[\begin{array}{l}
\theta_{\mathrm{c}} \widehat{\mathbf{A}}-\theta_{s} \widehat{\mathbf{Z}} \\
\theta_{s} \widehat{\mathbf{A}}+\theta_{\mathrm{c}} \widehat{\mathbf{Z}}
\end{array}\right]
$$

The use of the symbols $\widehat{\mathbf{B}}$ and $\widehat{\mathbf{W}}$ to represent $\widehat{\mathbf{A}}_{\theta}$ and $\widehat{\mathbf{Z}}_{\theta}$ is done for traditional reasons.

Multiplying (2.3-9) from the left by $\left[\begin{array}{rr}\theta_{\mathrm{c}} & \theta_{s} \\ -\theta_{s} & \theta_{\mathrm{c}}\end{array}\right]$, we obtain:

$$
\left[\begin{array}{l}
\widehat{\mathbf{A}} \\
\hat{\mathbf{z}}
\end{array}\right]=\left[\begin{array}{rr}
\theta_{\mathrm{c}} & \theta_{s} \\
-\theta_{s} & \theta_{\mathrm{c}}
\end{array}\right]\left[\begin{array}{c}
\widehat{\mathbf{B}} \\
\widehat{\mathbf{W}}
\end{array}\right]=\left[\begin{array}{l}
\theta_{s} \widehat{\mathbf{W}}+\theta_{\mathrm{c}} \widehat{\mathbf{B}} \\
\theta_{\mathrm{c}} \widehat{\mathbf{W}}-\theta_{s} \widehat{\mathbf{B}}
\end{array}\right]
$$

Using (2.3-5), we define the charge components along the $\widehat{\mathbf{Z}}, \widehat{\mathbf{A}}, \widehat{\mathbf{B}}$, and $\widehat{\mathbf{W}}$ directions, as follows:

$$
\overleftrightarrow{\mathrm{q}}_{z} \stackrel{\text { def }}{=} \widehat{\mathbf{Z}} \cdot \overleftrightarrow{\mathbf{q}} ; \quad \overleftrightarrow{\mathrm{q}}_{\mathrm{A}} \stackrel{\text { def }}{=} \widehat{\mathbf{A}} \cdot \overleftrightarrow{\mathbf{q}} ; \quad \overleftrightarrow{\mathrm{q}}_{\mathrm{B}} \stackrel{\text { def }}{=} \widehat{\mathbf{B}} \cdot \overleftrightarrow{\mathbf{q}} ; \quad \overleftrightarrow{\mathrm{q}}_{\mathrm{W}} \stackrel{\text { def }}{=} \widehat{\mathbf{W}} \cdot \overleftrightarrow{\mathbf{q}}
$$

Since by (2.3-9) we have $\widehat{\mathbf{B}}=\theta_{\mathrm{c}} \widehat{\mathbf{A}}-\theta_{s} \widehat{\mathbf{Z}}$ and $\widehat{\mathbf{W}}=\theta_{s} \widehat{\mathbf{A}}+\theta_{\mathrm{c}} \widehat{\mathbf{Z}}$, we conclude that:

$$
\overleftrightarrow{\mathrm{q}}_{\mathrm{B}}=\theta_{\mathrm{c}} \overleftrightarrow{\mathrm{q}}_{\mathrm{A}}-\theta_{s} \overleftrightarrow{\mathrm{q}}_{\mathrm{Z}} \quad ; \quad \stackrel{\mathrm{q}}{\mathrm{w}}_{\mathrm{w}}=\theta_{s} \overleftrightarrow{\mathrm{q}}_{\mathrm{A}}+\theta_{\mathrm{c}} \overleftrightarrow{\mathrm{q}}_{\mathrm{Z}}
$$

Here is the terminology that goes with these entities. We shall refer to $\widehat{\mathbf{Z}}$ as the Weak Charge Direction, and hence to $\overleftrightarrow{\mathrm{q}}_{z}$ as the Weak Charge. On the other hand, $\widehat{\mathbf{A}}$ will be referred to as the Electric Charge Direction, and hence $\overleftrightarrow{\mathrm{q}}_{\mathrm{A}}$ is the Electric Charge.

This suggests we should refer to the entire 2-dimensional charge space spanned by $\widehat{\mathbf{Z}}$ and $\widehat{\mathbf{A}}$ as the Electroweak Charge Plane. Since $\widehat{\mathbf{B}}$ and $\widehat{\mathbf{W}}$ are a mixture of $\widehat{\mathbf{Z}}$ and $\widehat{\mathbf{A}}$, they will be referred to collectively as the Electroweak Directions, and so $\overleftrightarrow{\mathrm{q}}_{\mathrm{B}}$ and $\overleftrightarrow{\mathrm{q}}_{\mathrm{w}}$ are called collectively Electroweak Charges.

The quantity $\theta_{s} \overleftrightarrow{\mathrm{q}}_{\mathrm{w}}$ is referred to as the Weak Isospin, however, UCV theory does not make any use of it. 


\section{Unified Charge Vectors Theory}

We recall that the charge quantization law (2.1-1) requires that:

$$
\overleftrightarrow{\mathbf{q}}=\widehat{\mathbf{Z}}\left(\frac{\widehat{\mathbf{Z}}_{\theta} \cdot \boldsymbol{s}}{\sqrt{3} \theta_{c}}+\frac{s_{0}-\overleftrightarrow{\Pi} s}{4 \theta_{c} \theta_{s}}\right)-\frac{s}{\sqrt{3}}
$$

Substituting this in (2.3-11), and using the fact that $\widehat{\mathbf{Z}} \cdot \widehat{\mathbf{Z}}=1$, and $\widehat{\mathbf{W}}=\hat{\mathbf{Z}}_{\theta}=\theta_{s} \widehat{\mathbf{A}}+\theta_{\mathrm{c}} \widehat{\mathbf{Z}}$, we conclude that:

$$
\overleftrightarrow{\mathrm{q}}_{z} \stackrel{\text { def }}{=} \hat{\mathbf{Z}} \cdot \overleftrightarrow{\mathbf{q}}=(\hat{\mathbf{Z}} \cdot \hat{\mathbf{Z}})\left(\frac{\widehat{\mathbf{W}} \cdot \boldsymbol{s}}{\sqrt{3} \theta_{c}}+\frac{s_{0}-\overleftrightarrow{\Pi}_{s}}{4 \theta_{c} \theta_{\mathrm{s}}}\right)-\frac{\widehat{\mathbf{Z}} \cdot \boldsymbol{s}}{\sqrt{3}}=\frac{\theta_{s} \widehat{\mathbf{A}} \cdot \boldsymbol{s}}{\sqrt{3} \theta_{c}}+\frac{s_{0}-\overleftrightarrow{\Pi}_{s}}{4 \theta_{c} \theta_{s}}
$$

However, since by (2.1-4) $\overleftarrow{\Pi}_{s} \neq \vec{\Pi} s$, we conclude that:

$$
\overleftarrow{q}_{z} \neq \vec{q}_{z}
$$

That is to say, the left and right charge vectors do not agree on their charge components along the $\widehat{\mathbf{Z}}$-direction.

Next, we compute $\overleftrightarrow{q}_{A}$. Using the fact that $\widehat{\mathbf{A}} \cdot \widehat{\mathbf{Z}}=0$, we conclude that:

$$
\overleftrightarrow{q}_{A} \stackrel{\text { def }}{=} \widehat{\mathbf{A}} \cdot \overleftrightarrow{\mathbf{q}}=(\widehat{\mathbf{A}} \cdot \widehat{\mathbf{Z}})\left(\frac{\widehat{\mathbf{W}} \cdot \boldsymbol{s}}{\sqrt{3} \theta_{\mathrm{c}}}+\frac{s_{0}-\overleftrightarrow{\Pi} \boldsymbol{s}}{4 \theta_{\mathrm{c}} \theta_{\mathrm{s}}}\right)-\frac{\widehat{\mathbf{A}} \cdot \boldsymbol{s}}{\sqrt{3}}=-\frac{\widehat{\mathbf{A}} \cdot \boldsymbol{s}}{\sqrt{3}}
$$

In particular, we notice that:

$$
\stackrel{\leftarrow}{q}_{A}=\vec{q}_{A}
$$

That is to say, the left and right charge vectors share the same electric component. This allows us to drop the upper arrows notation for $\overleftrightarrow{q}_{A}$ and simply write $q_{A}$ without any risk of ambiguity. 


\section{Unified Charge Vectors Theory}

\subsection{The Strong Charge Plane}

Since the charge space is 4-dimensional, we conclude that in addition to $\widehat{\mathbf{Z}}$ and $\widehat{\mathbf{A}}$, there must exist two more charge directions orthogonal both to each other and to the electroweak charge plane.

We shall refer to the charge plane orthogonal to the electroweak charge plane as the Strong Charge Plane. As in the case of the electroweak plane, we can choose any two arbitrary orthonormal vectors to serve as its basis.

While it is entirely possible to develop the theory of strong charges using only two orthonormal strong directions, the resulting equations obtain an artificially cumbersome form. As will become apparent later on when we discuss quarks, it will prove useful to use three strong directions rather than two. Since three vectors lying on a plane cannot possibly be orthogonal to each other, the best we can do is orient them evenly with a $120^{\circ}$ angle between each other, as shown in the following diagram:

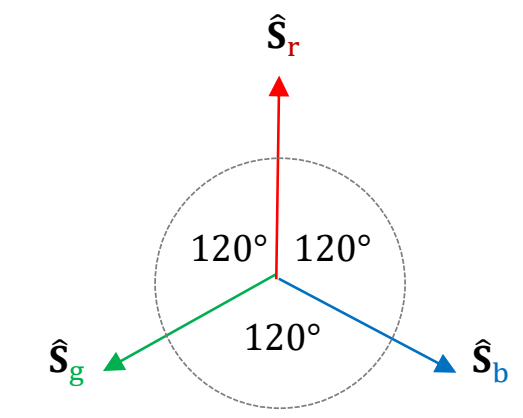

The three strong directions $\widehat{\mathbf{S}}_{\mathrm{r}}, \widehat{\mathbf{S}}_{\mathrm{g}}, \widehat{\mathbf{S}}_{\mathrm{b}}$

Care must be taken not to apply a 3D interpretation to this diagram and mistakenly think that these three vectors are orthogonal to each other in some 3-dimensional space. It is important to remember that we are dealing with three vectors lying on a 2-dimensional plane, with a $120^{\circ}$ angle between each other.

As the diagram's colors suggest, we refer to these three strong directions as, Red, Green, and Blue, and denote them respectively by $\widehat{\mathbf{S}}_{\mathrm{r}}, \widehat{\mathbf{S}}_{\mathrm{g}}$, and $\widehat{\mathbf{S}}_{\mathrm{b}}$. Here is the definition of this strong triplet of vectors which we will use:

$$
\widehat{\mathbf{S}}_{\mathrm{r}}=\widehat{\mathbf{S}}^{\mathrm{r}} \stackrel{\text { def }}{=} \frac{1}{\sqrt{6}}[0,-2,1,1] ; \quad \widehat{\mathbf{S}}_{\mathrm{g}}=\widehat{\mathbf{S}}^{\mathrm{g}} \stackrel{\text { def }}{=} \frac{1}{\sqrt{6}}[0,1,-2,1] ; \quad \widehat{\mathbf{S}}_{\mathrm{b}}=\widehat{\mathbf{S}}^{\mathrm{b}} \stackrel{\text { def }}{=} \frac{1}{\sqrt{6}}[0,1,1,-2]
$$

The reader is advised to take the time to verify that the above strong charge directions are indeed orthogonal to the electroweak plane, i.e., that:

$$
\widehat{\mathbf{S}}_{\mathrm{c}} \cdot \widehat{\mathbf{Z}}=\widehat{\mathbf{S}}_{\mathrm{c}} \cdot \widehat{\mathbf{A}}=0 \text { for } c \in\{\mathrm{r}, \mathrm{g}, \mathrm{b}\}
$$

Since $\widehat{\mathbf{S}}_{\mathrm{r}}, \widehat{\mathbf{S}}_{\mathrm{g}}$, and $\widehat{\mathbf{S}}_{\mathrm{b}}$ are linearly dependent, we can form a linear combination of them that vanishes. Indeed, we have: 


\section{Unified Charge Vectors Theory}

$$
\widehat{\mathbf{S}}_{\mathrm{r}}+\widehat{\mathbf{S}}_{\mathrm{g}}+\widehat{\mathbf{S}}_{\mathrm{b}}=0
$$

Computing the dot product of the strong vectors, we get:

$$
\widehat{\mathbf{S}}_{\mathrm{r}} \cdot \widehat{\mathbf{S}}_{\mathrm{r}}=\widehat{\mathbf{S}}_{\mathrm{g}} \cdot \widehat{\mathbf{S}}_{\mathrm{g}}=\widehat{\mathbf{S}}_{\mathrm{b}} \cdot \widehat{\mathbf{S}}_{\mathrm{b}}=1 \text { and } \hat{\mathbf{S}}_{\mathrm{r}} \cdot \widehat{\mathbf{S}}_{\mathrm{g}}=\widehat{\mathbf{S}}_{\mathrm{r}} \cdot \widehat{\mathbf{S}}_{\mathrm{b}}=\widehat{\mathbf{S}}_{\mathrm{b}} \cdot \widehat{\mathbf{S}}_{\mathrm{g}}=-\frac{1}{2}=\cos \left(120^{\circ}\right)
$$

That is to say, the projection of each of the vectors $\widehat{\mathbf{S}}_{\mathrm{r}}, \widehat{\mathbf{S}}_{\mathrm{g}}$, and $\widehat{\mathbf{S}}_{\mathrm{b}}$ on the others is negative, as can clearly be seen in figure (2.4-1). As we shall see, when we investigate particle interactions, the fact that the dot product of any two of the strong charge vectors is negative has a profound implication in the form of quarks attraction, enabling the existence of the proton.

Using the fact that $\widehat{\mathbf{S}}_{\mathrm{c}} \cdot \widehat{\mathbf{Z}}=0$, we compute the strong charges to be:

$$
\overleftrightarrow{q}_{c} \stackrel{\text { def }}{=} \widehat{\mathbf{S}}_{\mathrm{c}} \cdot \overleftrightarrow{\mathbf{q}}=\left(\widehat{\mathbf{S}}_{\mathrm{c}} \cdot \hat{\mathbf{Z}}\right)\left(\frac{\widehat{\mathbf{Z}}_{\theta} \cdot \boldsymbol{s}}{\sqrt{3} \theta_{\mathrm{c}}}+\frac{s_{0}-\overleftrightarrow{\Pi}_{s}}{4 \theta_{\mathrm{c}} \theta_{\mathrm{s}}}\right)-\frac{\widehat{\mathbf{S}}_{\mathrm{c}} \cdot \boldsymbol{s}}{\sqrt{3}}=-\frac{\widehat{\mathbf{S}}_{\mathrm{c}} \cdot \boldsymbol{s}}{\sqrt{3}} \quad \text { for } c \in\{\mathrm{r}, \mathrm{g}, \mathrm{b}\}
$$

As in the case of the electric charge, we see that:

$$
\overleftarrow{q}_{c}=\vec{q}_{c}
$$

And so, we can unambiguously drop the upper arrow notation and simply write $q_{c}$. We shall refer to $q_{\mathrm{r}}, q_{\mathrm{g}}$, and $q_{\mathrm{b}}$, respectively, as the Red, Green, and Blue Strong Charges. 


\section{Unified Charge Vectors Theory}

Though the three directions $\widehat{\mathbf{S}}^{\mathrm{r}}, \widehat{\mathbf{S}}^{\mathrm{g}}$, and $\widehat{\mathbf{S}}^{\mathrm{b}}$ result in simplified equations, we shall also need later on to make use of some two orthogonal directions in the strong plane. Any two such directions will do the job, so we take the liberty to set them arbitrarily as follows:

$$
\widehat{\mathbf{G}}^{\perp} \stackrel{\text { def }}{=} \widehat{\mathbf{S}}^{r} \text { and } \widehat{\mathbf{G}}^{\vdash} \stackrel{\text { def }}{=} \frac{1}{\sqrt{3}}\left(\widehat{\mathbf{S}}^{\mathrm{b}}-\widehat{\mathbf{S}}^{\mathrm{g}}\right)
$$

The following diagram depicts the relations between these five directions:

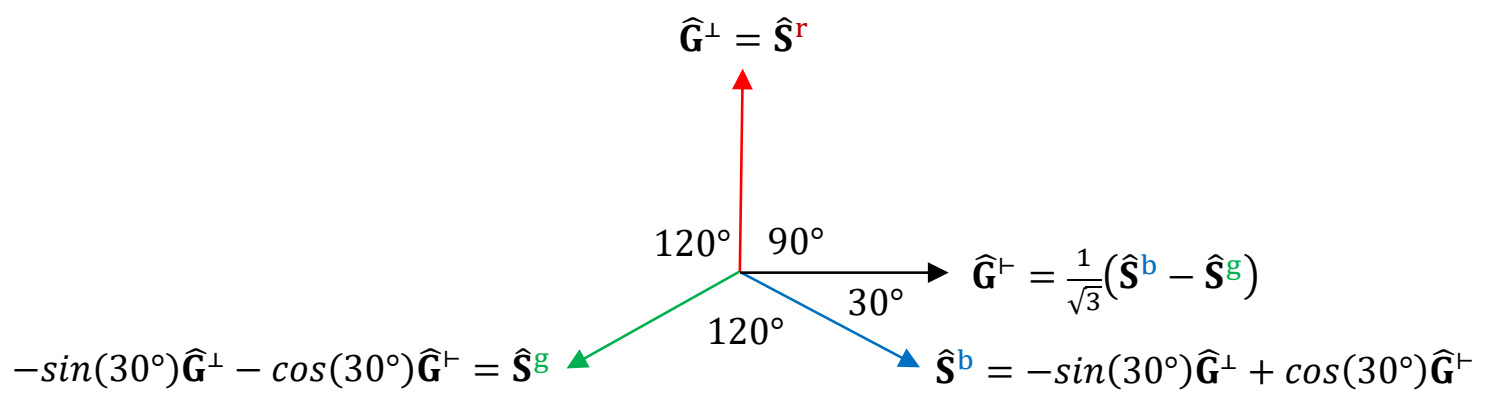

The five strong directions $\widehat{\mathbf{G}}^{\perp}, \widehat{\mathbf{G}}^{\vdash}, \widehat{\mathbf{S}}^{\mathrm{r}}, \widehat{\mathbf{S}}^{\mathrm{g}}, \widehat{\mathbf{S}}^{\mathrm{b}}$

This implies that:

$$
\widehat{\mathbf{G}}^{\perp} \cdot \widehat{\mathbf{G}}^{\perp}=1 ; \quad \widehat{\mathbf{G}}^{\vdash} \cdot \widehat{\mathbf{G}}^{\vdash}=\frac{1}{3}\left(\widehat{\mathbf{S}}^{\mathrm{b}} \cdot \widehat{\mathbf{S}}^{\mathrm{b}}-2 \widehat{\mathbf{S}}^{\mathrm{b}} \cdot \widehat{\mathbf{S}}^{\mathrm{g}}+\widehat{\mathbf{S}}^{\mathrm{g}} \cdot \widehat{\mathbf{S}}^{\mathrm{g}}\right)=1 ; \quad \widehat{\mathbf{G}}^{\perp} \cdot \widehat{\mathbf{G}}^{\vdash}=0
$$

And:

$$
\left[\begin{array}{c}
\widehat{\mathbf{G}}^{\perp} \cdot \widehat{\mathbf{S}}^{\mathrm{r}} \\
\widehat{\mathbf{G}}^{\perp} \cdot \widehat{\mathbf{S}}^{\mathrm{g}} \\
\widehat{\mathbf{G}}^{\perp} \cdot \widehat{\mathbf{S}}^{\mathrm{b}}
\end{array}\right]=\left[\begin{array}{c}
+1 \\
-\frac{1}{2} \\
-\frac{1}{2}
\end{array}\right] \quad \text { and }\left[\begin{array}{c}
\widehat{\mathbf{G}}^{\vdash} \cdot \widehat{\mathbf{S}}^{\mathrm{r}} \\
\widehat{\mathbf{G}}^{\vdash} \cdot \widehat{\mathbf{S}}^{\mathrm{g}} \\
\widehat{\mathbf{G}}^{\vdash} \cdot \widehat{\mathbf{S}}^{\mathrm{b}}
\end{array}\right]=\left[\begin{array}{c}
0 \\
-\frac{\sqrt{3}}{2} \\
+\frac{\sqrt{3}}{2}
\end{array}\right]
$$

Hence:

$$
\begin{gathered}
{\left[\begin{array}{l}
\widehat{\mathbf{S}}^{\mathrm{r}} \\
\widehat{\mathbf{S}}^{\mathrm{g}} \\
\widehat{\mathbf{S}}^{\mathrm{b}}
\end{array}\right]=\left[\begin{array}{ll}
\widehat{\mathbf{S}}^{\mathrm{r}} \cdot \widehat{\mathbf{G}}^{\perp} \\
\widehat{\mathbf{S}}^{\mathrm{g}} \cdot \widehat{\mathbf{G}}^{\perp} \\
\widehat{\mathbf{S}}^{\mathrm{b}} \cdot \widehat{\mathbf{G}}^{\perp}
\end{array}\right] \widehat{\mathbf{G}}^{\perp}+\left[\begin{array}{l}
\widehat{\mathbf{S}}^{\mathrm{r}} \cdot \widehat{\mathbf{G}}^{\vdash} \\
\widehat{\mathbf{S}}^{\mathrm{g}} \cdot \widehat{\mathbf{G}}^{\vdash} \\
\widehat{\mathbf{S}}^{\mathrm{b}} \cdot \widehat{\mathbf{G}}^{\vdash}
\end{array}\right] \widehat{\mathbf{G}}^{\vdash}=\frac{1}{2}\left[\begin{array}{l}
+2 \\
-1 \\
-1
\end{array}\right] \widehat{\mathbf{G}}^{\perp}+\frac{\sqrt{3}}{2}\left[\begin{array}{r}
0 \\
-1 \\
+1
\end{array}\right] \widehat{\mathbf{G}}^{\vdash}} \\
{\left[\begin{array}{ccc}
\widehat{\mathbf{S}}^{\mathrm{r}} & 0 & 0 \\
0 & \widehat{\mathbf{S}}^{\mathrm{g}} & 0 \\
0 & 0 & \widehat{\mathbf{S}}^{\mathrm{b}}
\end{array}\right]=\frac{\sqrt{3}}{2}\left[\begin{array}{ccc}
\frac{2}{\sqrt{3}} \widehat{\mathbf{G}}^{\perp} & 0 \\
0 & -\frac{1}{\sqrt{3}} \widehat{\mathbf{G}}^{\perp}-\widehat{\mathbf{G}}^{\vdash} & 0 \\
0 & 0 & -\frac{1}{\sqrt{3}} \widehat{\mathbf{G}}^{\perp}+\widehat{\mathbf{G}}^{\vdash}
\end{array}\right]}
\end{gathered}
$$

Equation (2.4-13) will prove extremely useful later on when we compute the unified Lagrangian. 


\section{Unified Charge Vectors Theory}

\subsection{Anti-Particles}

In this section, we want to develop the relation between the charge vectors of particles and anti-particles.

We recall that by table (2.2-1), the relation between the signatures of anti-particles and particles is given by:

$$
\overline{8}=-s \text { so } \overleftrightarrow{\Pi} \bar{s}=\overleftrightarrow{\Pi} s
$$

Substituting this in the charge quantization equation, we obtain the following result:

$$
\overleftrightarrow{\mathbf{q}}=\hat{\mathbf{Z}}\left(\frac{\widehat{\mathbf{W}} \cdot \overline{\boldsymbol{s}}}{\sqrt{3} \theta_{\mathrm{c}}}+\frac{\bar{s}_{0}-\overleftrightarrow{\Pi} \overline{\boldsymbol{s}}}{4 \theta_{\mathrm{c}} \theta_{\mathrm{s}}}\right)-\frac{\overline{\boldsymbol{s}}}{\sqrt{3}}=-\left(\hat{\mathbf{Z}}\left(\frac{\widehat{\mathbf{W}} \cdot \boldsymbol{s}}{\sqrt{3} \theta_{\mathrm{c}}}+\frac{s_{0}+\overleftrightarrow{\Pi} \boldsymbol{s}}{4 \theta_{\mathrm{c}} \theta_{\mathrm{s}}}\right)-\frac{\boldsymbol{s}}{\sqrt{3}}\right)
$$

Using the fact that $\overleftarrow{\Pi}_{s}=-\vec{\Pi} s$, we can rewrite (2.5-2) as follows:

$$
\begin{aligned}
& \overleftarrow{\overline{\mathrm{q}}}=-\left(\hat{\mathbf{z}}\left(\frac{\widehat{\mathbf{W}} \cdot \boldsymbol{s}}{\sqrt{3} \theta_{\mathrm{c}}}+\frac{s_{0}-\vec{\Pi} s}{4 \theta_{\mathrm{c}} \theta_{\mathrm{s}}}\right)-\frac{\boldsymbol{s}}{\sqrt{3}}\right)=-\overrightarrow{\mathbf{q}} \\
& \overrightarrow{\mathbf{q}}=-\left(\hat{\mathbf{z}}\left(\frac{\widehat{\mathbf{W}} \cdot \boldsymbol{s}}{\sqrt{3} \theta_{\mathrm{c}}}+\frac{s_{0}-\overleftarrow{\Pi} s}{4 \theta_{\mathrm{c}} \theta_{\mathrm{s}}}\right)-\frac{\boldsymbol{s}}{\sqrt{3}}\right)=-\overleftarrow{\mathrm{q}}
\end{aligned}
$$

That is to say, to obtain the charge vectors of an anti-particle, we have to reverse the signature, as well as flip between left and right. 


\section{Unified Charge Vectors Theory}

\subsection{The Neutrinos}

Row \#1 in table (2.2-1) tells us that neutrinos' signature satisfies:

$$
\boldsymbol{s}^{v} \stackrel{\text { def }}{=}[1,1,1,1] \text { so } s_{0}^{v}=1 \text { and } \overleftarrow{\Pi} s^{v}=-1 \text { and } \vec{\Pi} s^{v}=1
$$

We also have:

$$
\begin{aligned}
& \boldsymbol{s}^{v}=2 \widehat{\mathbf{Z}} \text { so } \hat{\mathbf{Z}} \cdot \boldsymbol{s}^{v}=2 \text { and } \widehat{\mathbf{A}} \cdot \boldsymbol{s}^{v}=0 \text { and } \widehat{\mathbf{S}}_{\mathrm{c}} \cdot \boldsymbol{s}^{v}=0 \\
& \text { so } \widehat{\mathbf{W}} \cdot \boldsymbol{s}^{v}=\left(\theta_{\mathrm{S}} \widehat{\mathbf{A}}+\theta_{\mathrm{c}} \widehat{\mathbf{z}}\right) \cdot \boldsymbol{s}^{v}=2 \theta_{\mathrm{c}}
\end{aligned}
$$

Computing the weak charges, we conclude that for neutrinos, we have:

$$
\begin{aligned}
& \overleftarrow{q}_{z}^{v} \stackrel{\text { def }}{=} \widehat{\mathbf{Z}} \cdot \overleftarrow{\mathrm{q}}^{v}=\widehat{\mathbf{Z}} \cdot\left(\widehat{\mathbf{Z}}\left(\frac{\widehat{\mathbf{W}} \cdot \boldsymbol{s}^{v}}{\sqrt{3} \theta_{\mathrm{c}}}+\frac{s_{0}-\overleftarrow{\Pi}_{s^{v}}}{4 \theta_{\mathrm{c}} \theta_{\mathrm{s}}}\right)-\frac{\boldsymbol{s}^{v}}{\sqrt{3}}\right)=\frac{1}{2 \theta_{\mathrm{c}} \theta_{s}} \\
& \vec{q}_{z}^{v} \stackrel{\text { def }}{=} \hat{\mathbf{Z}} \cdot \overrightarrow{\mathbf{q}}^{v}=\hat{\mathbf{Z}} \cdot\left(\widehat{\mathbf{Z}}\left(\frac{\widehat{\mathbf{W}} \cdot \boldsymbol{s}^{v}}{\sqrt{3} \theta_{\mathrm{c}}}+\frac{s_{0}-\vec{\Pi} s^{v}}{4 \theta_{\mathrm{c}} \theta_{\mathrm{s}}}\right)-\frac{\boldsymbol{s}^{v}}{\sqrt{3}}\right)=0
\end{aligned}
$$

And for the electric and strong charges, we have:

$$
\begin{aligned}
& q_{A}^{v} \stackrel{\text { def }}{=} \widehat{\mathbf{A}} \cdot \overleftrightarrow{\mathbf{q}}^{v}=\widehat{\mathbf{A}} \cdot\left(\widehat{\mathbf{Z}}\left(\frac{\widehat{\mathbf{W}} \cdot \boldsymbol{s}^{v}}{\sqrt{3} \theta_{\mathrm{c}}}+\frac{s_{0}-\overleftrightarrow{\Pi} \boldsymbol{s}^{v}}{4 \theta_{\mathrm{c}} \theta_{\mathrm{s}}}\right)-\frac{\boldsymbol{s}^{v}}{\sqrt{3}}\right)=0 \\
& q_{c}^{v} \stackrel{\text { def }}{=} \widehat{\mathbf{S}}_{\mathrm{c}} \cdot \overleftrightarrow{\mathbf{q}}^{v}=\widehat{\mathbf{S}}_{\mathrm{c}} \cdot\left(\widehat{\mathbf{Z}}\left(\frac{\widehat{\mathbf{W}} \cdot \boldsymbol{s}^{v}}{\sqrt{3} \theta_{\mathrm{c}}}+\frac{s_{0}-\overleftrightarrow{\Pi}_{s^{v}}}{4 \theta_{\mathrm{c}} \theta_{\mathrm{s}}}\right)-\frac{\boldsymbol{s}^{v}}{\sqrt{3}}\right)=0
\end{aligned}
$$

Hence, we conclude that neutrinos carry weak charge only. Using (2.3-12), we compute:

$$
\begin{aligned}
& \grave{q}_{B}^{v}=\theta_{\mathrm{c}} \stackrel{\leftrightarrow}{q}_{A}^{v}-\theta_{s} \overleftarrow{q}_{Z}^{v}=-\frac{1}{2 \theta_{\mathrm{c}}} \quad \text { and } \quad \vec{q}_{B}^{v}=\theta_{\mathrm{c}} \vec{q}_{A}^{v}-\theta_{S} \vec{q}_{Z}^{v}=0 \\
& \grave{q}_{W}^{v}=\theta_{s} \stackrel{q}{q}_{A}^{v}+\theta_{\mathrm{c}} \grave{q}_{Z}^{v}=\frac{1}{2 \theta_{s}} \quad \text { and } \quad \vec{q}_{W}^{v}=\theta_{s} \vec{q}_{A}^{v}+\theta_{\mathrm{c}} \vec{q}_{Z}^{v}=0
\end{aligned}
$$

Hence, we conclude that:

$$
\begin{aligned}
\overleftarrow{\mathbf{q}}^{v}=\frac{\widehat{\mathbf{z}}}{2 \theta_{\mathrm{c}} \theta_{s}} & =-\frac{\widehat{\mathbf{B}}}{2 \theta_{\mathrm{c}}}+\frac{\widehat{\mathbf{W}}}{2 \theta_{\mathrm{s}}} \\
\overrightarrow{\mathbf{q}}^{v} & =0
\end{aligned}
$$




\section{Unified Charge Vectors Theory}

\subsection{The Electrons}

Row \#5 in table (2.2-1) tells us that Electrons' signature satisfies:

$$
\boldsymbol{s}^{e} \stackrel{\text { def }}{=}[-1,1,1,1] \text { so } s_{0}^{e}=-1 \text { and } \overleftarrow{\Pi}_{s}{ }^{e}=1 \text { and } \vec{\Pi}_{s^{e}}=-1
$$

We also have:

$$
\begin{gathered}
\boldsymbol{s}^{e}=\widehat{\mathbf{Z}}+\sqrt{3} \widehat{\mathbf{A}} \text { so } \hat{\mathbf{Z}} \cdot \boldsymbol{s}^{e}=1 \text { and } \widehat{\mathbf{A}} \cdot \boldsymbol{s}^{e}=\sqrt{3} \text { and } \widehat{\mathbf{S}}_{\mathrm{c}} \cdot \boldsymbol{s}^{e}=0 \\
\text { so } \widehat{\mathbf{W}} \cdot \boldsymbol{s}^{e}=\left(\theta_{\mathrm{s}} \widehat{\mathbf{A}}+\theta_{\mathrm{c}} \widehat{\mathbf{Z}}\right) \cdot \boldsymbol{s}^{e}=\theta_{\mathrm{s}} \sqrt{3}+\theta_{\mathrm{c}}
\end{gathered}
$$

Computing the weak charge, we conclude that for electrons, we have:

$$
\begin{aligned}
& \overleftarrow{q}_{z}^{e} \stackrel{\text { def }}{=} \hat{\mathbf{Z}} \cdot \overleftarrow{\mathrm{q}}^{e}=\hat{\mathbf{Z}} \cdot\left(\hat{\mathbf{Z}}\left(\frac{\widehat{\mathbf{W}} \cdot \boldsymbol{s}^{e}}{\sqrt{3} \theta_{\mathrm{c}}}+\frac{s_{0}-\overleftarrow{\Pi} s^{e}}{4 \theta_{\mathrm{c}} \theta_{\mathrm{s}}}\right)-\frac{\boldsymbol{s}^{e}}{\sqrt{3}}\right)=\frac{\theta_{s}}{\theta_{\mathrm{c}}}-\frac{1}{2 \theta_{\mathrm{c}} \theta_{s}} \\
& \vec{q}_{z}^{e} \stackrel{\text { def }}{=} \hat{\mathbf{Z}} \cdot \overrightarrow{\mathbf{q}}^{e}=\hat{\mathbf{Z}} \cdot\left(\hat{\mathbf{Z}}\left(\frac{\widehat{\mathbf{W}} \cdot \boldsymbol{s}^{e}}{\sqrt{3} \theta_{\mathrm{c}}}+\frac{s_{0}-\vec{\Pi} s^{e}}{4 \theta_{\mathrm{c}} \theta_{\mathrm{s}}}\right)-\frac{\boldsymbol{s}^{e}}{\sqrt{3}}\right)=\frac{\theta_{s}}{\theta_{\mathrm{c}}}
\end{aligned}
$$

And for the electric and strong charges, we have:

$$
\begin{gathered}
q_{\mathrm{A}}^{e} \stackrel{\text { def }}{=} \widehat{\mathbf{A}} \cdot \overleftrightarrow{\mathbf{q}}^{e}=\widehat{\mathbf{A}} \cdot\left(\hat{\mathbf{Z}}\left(\frac{\widehat{\mathbf{W}} \cdot \boldsymbol{s}^{e}}{\sqrt{3} \theta_{\mathrm{c}}}+\frac{s_{0}-\overleftrightarrow{\Pi} s^{\nu}}{4 \theta_{\mathrm{c}} \theta_{\mathrm{s}}}\right)-\frac{\boldsymbol{s}^{e}}{\sqrt{3}}\right)=-1 \\
q_{c}^{e} \stackrel{\text { def }}{=} \widehat{\mathbf{S}}_{\mathrm{c}} \cdot \overleftrightarrow{\mathbf{q}}^{e}=\widehat{\mathbf{S}}_{\mathrm{c}} \cdot\left(\widehat{\mathbf{Z}}\left(\frac{\widehat{\mathbf{W}} \cdot \boldsymbol{s}^{e}}{\sqrt{3} \theta_{\mathrm{c}}}+\frac{s_{0}-\overleftrightarrow{\Pi} \boldsymbol{s}^{\nu}}{4 \theta_{\mathrm{c}} \theta_{\mathrm{s}}}\right)-\frac{\boldsymbol{s}^{e}}{\sqrt{3}}\right)=0
\end{gathered}
$$

Hence, we see that electrons carry electroweak charges only. Using (2.3-12), we compute:

$$
\begin{aligned}
& \grave{q}_{B}^{e}=\theta_{\mathrm{c}} \overleftarrow{q}_{A}^{e}-\theta_{s} \overleftarrow{q}_{Z}^{e}=-\frac{1}{2 \theta_{\mathrm{c}}} \quad \text { and } \quad \vec{q}_{B}^{e}=\theta_{\mathrm{c}} \vec{q}_{A}^{e}-\theta_{s} \vec{q}_{Z}^{e}=-\frac{1}{\theta_{\mathrm{c}}} \\
& \overleftarrow{q}_{W}^{e}=\theta_{s} \overleftarrow{q}_{A}^{e}+\theta_{\mathrm{c}} \overleftarrow{q}_{Z}^{e}=-\frac{1}{2 \theta_{\mathrm{s}}} \quad \text { and } \quad \vec{q}_{W}^{e}=\theta_{S} \vec{q}_{A}^{e}+\theta_{\mathrm{c}} \vec{q}_{Z}^{e}=0
\end{aligned}
$$

Hence, we conclude that:

$$
\begin{aligned}
& \overleftarrow{\mathbf{q}}^{e}=\widehat{\mathbf{Z}}\left(\frac{\theta_{s}}{\theta_{\mathrm{c}}}-\frac{1}{2 \theta_{\mathrm{c}} \theta_{s}}\right)-\widehat{\mathbf{A}}=-\frac{\widehat{\mathbf{B}}}{2 \theta_{\mathrm{c}}}-\frac{\widehat{\mathbf{W}}}{2 \theta_{\mathrm{s}}} \\
& \overrightarrow{\mathbf{q}}^{e}=\widehat{\mathbf{Z}} \frac{\theta_{s}}{\theta_{\mathrm{c}}}-\widehat{\mathbf{A}}=-\frac{\widehat{\mathbf{B}}}{\theta_{\mathrm{c}}}
\end{aligned}
$$




\section{Unified Charge Vectors Theory}

\subsection{The Dark Quarks}

Row \#2 in table (2.2-1) tells us that Dark Red Quarks' signature satisfies:

$$
\boldsymbol{s}^{\mathrm{r}} \stackrel{\text { def }}{=}[1,1,-1,-1] \text { so } s_{0}^{\mathrm{r}}=1 \text { and } \overleftarrow{\Pi} s^{\mathrm{r}}=-1 \text { and } \vec{\Pi} s^{\mathrm{r}}=1
$$

We also have:

$$
\begin{gathered}
\boldsymbol{s}^{\mathrm{r}}=-\frac{2}{\sqrt{3}} \widehat{\mathbf{A}}-\frac{2 \sqrt{2}}{\sqrt{3}} \widehat{\mathbf{S}}_{\mathrm{r}} \text { so } \hat{\mathbf{Z}} \cdot \boldsymbol{s}^{\mathrm{r}}=0 \text { and } \widehat{\mathbf{A}} \cdot \boldsymbol{s}^{\mathrm{r}}=-\frac{2}{\sqrt{3}} \text { and } \widehat{\mathbf{S}}_{\mathrm{r}} \cdot \boldsymbol{s}^{\mathrm{r}}=-\frac{2 \sqrt{2}}{\sqrt{3}} \\
\text { and } \widehat{\mathbf{W}} \cdot \boldsymbol{s}^{\mathrm{r}}=\left(\theta_{\mathrm{s}} \widehat{\mathbf{A}}+\theta_{\mathrm{c}} \hat{\mathbf{Z}}\right) \cdot \boldsymbol{s}^{\mathrm{r}}=-\frac{2 \theta_{\mathrm{s}}}{\sqrt{3}}
\end{gathered}
$$

Computing the weak charges, we see that for dark red quarks, we have:

$$
\begin{aligned}
& \grave{q}_{z}^{\mathrm{r}} \stackrel{\text { def }}{=} \hat{\mathbf{Z}} \cdot \overleftarrow{\mathrm{q}}^{\mathrm{r}}=\hat{\mathbf{Z}} \cdot\left(\widehat{\mathbf{Z}}\left(\frac{\widehat{\mathbf{W}} \cdot \boldsymbol{s}^{\mathrm{r}}}{\sqrt{3} \theta_{\mathrm{c}}}+\frac{s_{0}-\overleftarrow{\Pi}_{s^{\mathrm{r}}}}{4 \theta_{\mathrm{c}} \theta_{\mathrm{s}}}\right)-\frac{\boldsymbol{s}^{\mathrm{r}}}{\sqrt{3}}\right)=-\frac{2 \theta_{s}}{3 \theta_{\mathrm{c}}}+\frac{1}{2 \theta_{\mathrm{c}} \theta_{s}} \\
& \vec{q}_{z}^{\mathrm{r}} \stackrel{\text { def }}{=} \hat{\mathbf{Z}} \cdot \overrightarrow{\mathbf{q}}^{\mathrm{r}}=\hat{\mathbf{Z}} \cdot\left(\hat{\mathbf{Z}}\left(\frac{\widehat{\mathbf{W}} \cdot \boldsymbol{s}^{\mathrm{r}}}{\sqrt{3} \theta_{\mathrm{c}}}+\frac{s_{0}-\vec{\Pi}_{s^{\mathrm{r}}}}{4 \theta_{\mathrm{c}} \theta_{\mathrm{s}}}\right)-\frac{\boldsymbol{s}^{\mathrm{r}}}{\sqrt{3}}\right)=-\frac{2 \theta_{s}}{3 \theta_{\mathrm{c}}}
\end{aligned}
$$

And for the electric and strong charges, we have:

$$
\begin{aligned}
& q_{\mathrm{A}}^{\mathrm{r}} \stackrel{\text { def }}{=} \widehat{\mathbf{A}} \cdot \overleftrightarrow{\mathbf{q}}^{\mathrm{r}}=\widehat{\mathbf{A}} \cdot\left(\hat{\mathbf{Z}}\left(\frac{\widehat{\mathbf{W}} \cdot \boldsymbol{s}^{\mathrm{r}}}{\sqrt{3} \theta_{\mathrm{c}}}+\frac{s_{0}-\overleftrightarrow{\Pi} \boldsymbol{s}^{\mathrm{r}}}{4 \theta_{\mathrm{c}} \theta_{\mathrm{s}}}\right)-\frac{\boldsymbol{s}^{\mathrm{r}}}{\sqrt{3}}\right)=\frac{2}{3} \\
& q_{\mathrm{r}}^{\mathrm{r}} \stackrel{\text { def }}{=} \widehat{\mathbf{S}}_{\mathrm{r}} \cdot \overleftrightarrow{\mathbf{q}}^{\mathrm{r}}=\widehat{\mathbf{S}}_{\mathrm{r}} \cdot\left(\widehat{\mathbf{Z}}\left(\frac{\widehat{\mathbf{W}} \cdot \boldsymbol{s}^{\mathrm{r}}}{\sqrt{3} \theta_{\mathrm{c}}}+\frac{s_{0}-\overleftrightarrow{\Pi} \boldsymbol{s}^{\mathrm{r}}}{4 \theta_{\mathrm{c}} \theta_{\mathrm{s}}}\right)-\frac{\boldsymbol{s}^{\mathrm{r}}}{\sqrt{3}}\right)=\frac{2 \sqrt{2}}{3}
\end{aligned}
$$




\section{Unified Charge Vectors Theory}

We, therefore, see that dark-quarks carry electroweak and strong charges. Using (2.3-12), we compute:

$$
\begin{aligned}
& \stackrel{\leftarrow}{q}_{B}^{\mathrm{r}}=\theta_{\mathrm{c}} \stackrel{\leftrightarrow}{q}_{A}^{\mathrm{r}}-\theta_{s} \stackrel{\leftrightarrow}{q}_{Z}^{\mathrm{r}}=\frac{1}{6 \theta_{\mathrm{c}}} \quad \text { and } \quad \vec{q}_{B}^{\mathrm{r}}=\theta_{\mathrm{c}} \vec{q}_{A}^{\mathrm{r}}-\theta_{S} \vec{q}_{Z}^{\mathrm{r}}=\frac{2}{3 \theta_{\mathrm{c}}} \\
& \grave{q}_{W}^{\mathrm{r}}=\theta_{s} \overleftarrow{q}_{A}^{\mathrm{r}}+\theta_{\mathrm{c}} \stackrel{\leftarrow}{q}_{Z}^{\mathrm{r}}=\frac{1}{2 \theta_{\mathrm{s}}} \quad \text { and } \quad \vec{q}_{W}^{\mathrm{r}}=\theta_{s} \vec{q}_{A}^{\mathrm{r}}+\theta_{\mathrm{c}} \vec{q}_{Z}^{\mathrm{r}}=0
\end{aligned}
$$

Repeating the same computation for dark green and blue quarks, we conclude that:

$$
\begin{aligned}
& \overleftarrow{\mathbf{q}}^{c}=\widehat{\mathbf{Z}}\left(\frac{1}{2 \theta_{\mathrm{c}} \theta_{s}}-\frac{2 \theta_{s}}{3 \theta_{\mathrm{c}}}\right)+\widehat{\mathbf{A}} \frac{2}{3}+\frac{2 \sqrt{2}}{3} \widehat{\mathbf{S}}^{\mathrm{c}}=\frac{\widehat{\mathbf{B}}}{6 \theta_{\mathrm{c}}}+\frac{\widehat{\mathbf{W}}}{2 \theta_{\mathrm{s}}}+\frac{2 \sqrt{2}}{3} \widehat{\mathbf{S}}^{\mathrm{c}} \\
& \overrightarrow{\mathbf{q}}^{\mathrm{c}}=-\widehat{\mathbf{Z}} \frac{2 \theta_{s}}{3 \theta_{\mathrm{c}}}+\widehat{\mathbf{A}} \frac{2}{3}+\frac{2 \sqrt{2}}{3} \widehat{\mathbf{S}}^{\mathrm{c}}=\frac{2 \widehat{\mathbf{B}}}{3 \theta_{\mathrm{c}}}+\frac{2 \sqrt{2}}{3} \widehat{\mathbf{S}}^{\mathrm{c}}
\end{aligned}
$$




\section{Unified Charge Vectors Theory}

\subsection{The Bright Quarks}

Row \#6 in table (2.2-1) tells us that bright red quarks' signature satisfies:

$$
\boldsymbol{s}^{\mathrm{R}} \stackrel{\text { def }}{=}[-1,+1,-1,-1] \text { so } s_{0}^{\mathrm{R}}=-1 \text { and } \overleftarrow{\Pi}_{s}^{\mathrm{R}}=1 \text { and } \vec{\Pi}_{s^{\mathrm{R}}}=-1
$$

Where capital color latter (e.g., R) indicates a bright quark. We also have:

$$
\begin{gathered}
\boldsymbol{s}^{\mathrm{R}}=-\widehat{\mathbf{Z}}+\frac{1}{\sqrt{3}} \widehat{\mathbf{A}}-\frac{2 \sqrt{2}}{\sqrt{3}} \widehat{\mathbf{S}}^{\mathrm{R}} \text { so } \hat{\mathbf{Z}} \cdot \boldsymbol{s}^{\mathrm{R}}=-1 \text { and } \widehat{\mathbf{A}} \cdot \boldsymbol{s}^{\mathrm{R}}=\frac{1}{\sqrt{3}} \text { and } \widehat{\mathbf{S}}_{\mathrm{r}} \cdot \boldsymbol{s}^{\mathrm{R}}=-\frac{2 \sqrt{2}}{\sqrt{3}} \\
\text { and } \widehat{\mathbf{W}} \cdot \boldsymbol{s}^{\mathrm{R}}=\left(\theta_{\mathrm{s}} \widehat{\mathbf{A}}+\theta_{\mathrm{c}} \widehat{\mathbf{Z}}\right) \cdot \boldsymbol{s}^{\mathrm{R}}=\frac{\theta_{\mathrm{s}}}{\sqrt{3}}-\theta_{\mathrm{c}}
\end{gathered}
$$

Computing the weak charges, we get for bright red quarks:

$$
\begin{aligned}
& \overleftarrow{q}_{z}^{\mathrm{R}} \stackrel{\text { def }}{=} \hat{\mathbf{Z}} \cdot \overleftarrow{q}^{\mathrm{R}}=\hat{\mathbf{Z}} \cdot\left(\hat{\mathbf{Z}}\left(\frac{\widehat{\mathbf{W}} \cdot \boldsymbol{s}^{\mathrm{R}}}{\sqrt{3} \theta_{\mathrm{c}}}+\frac{s_{0}-\overleftarrow{\Pi}_{s^{\mathrm{R}}}^{\mathrm{R}}}{4 \theta_{\mathrm{c}} \theta_{\mathrm{s}}}\right)-\frac{\boldsymbol{s}^{\mathrm{R}}}{\sqrt{3}}\right)=\frac{\theta_{s}}{3 \theta_{\mathrm{c}}}-\frac{1}{2 \theta_{\mathrm{c}} \theta_{s}} \\
& \vec{q}_{z}^{\mathrm{R}} \stackrel{\text { def }}{=} \hat{\mathbf{Z}} \cdot \overrightarrow{\mathbf{q}}^{\mathrm{R}}=\hat{\mathbf{Z}} \cdot\left(\hat{\mathbf{Z}}\left(\frac{\widehat{\mathbf{W}} \cdot \boldsymbol{s}^{\mathrm{R}}}{\sqrt{3} \theta_{\mathrm{c}}}+\frac{s_{0}-\vec{\Pi}_{s^{\mathrm{R}}}}{4 \theta_{\mathrm{c}} \theta_{\mathrm{s}}}\right)-\frac{\boldsymbol{s}^{\mathrm{R}}}{\sqrt{3}}\right)=\frac{\theta_{s}}{3 \theta_{\mathrm{c}}}
\end{aligned}
$$

And for the electric and strong charges, we have:

$$
\begin{aligned}
& q_{A}^{\mathrm{R}} \stackrel{\text { def }}{=} \widehat{\mathbf{A}} \cdot \overleftrightarrow{\mathbf{q}}^{\mathrm{R}}=\widehat{\mathbf{A}} \cdot\left(\widehat{\mathbf{Z}}\left(\frac{\widehat{\mathbf{W}} \cdot \boldsymbol{s}^{\mathrm{R}}}{\sqrt{3} \theta_{\mathrm{c}}}+\frac{s_{0}-\overleftrightarrow{\Pi} s^{\mathrm{R}}}{4 \theta_{\mathrm{c}} \theta_{\mathrm{s}}}\right)-\frac{\boldsymbol{s}^{\mathrm{R}}}{\sqrt{3}}\right)=-\frac{1}{3} \\
& q_{\mathrm{r}}^{\mathrm{R}} \stackrel{\text { def }}{=} \widehat{\mathbf{S}}_{\mathrm{r}} \cdot \overleftrightarrow{\mathbf{q}}^{\mathrm{R}}=\widehat{\mathbf{S}}_{\mathrm{r}} \cdot\left(\hat{\mathbf{Z}}\left(\frac{\widehat{\mathbf{W}} \cdot \boldsymbol{s}^{\mathrm{R}}}{\sqrt{3} \theta_{\mathrm{c}}}+\frac{s_{0}-\overleftrightarrow{\Pi} s^{\mathrm{R}}}{4 \theta_{\mathrm{c}} \theta_{\mathrm{s}}}\right)-\frac{\boldsymbol{s}^{\mathrm{R}}}{\sqrt{3}}\right)=\frac{2 \sqrt{2}}{3}
\end{aligned}
$$

Hence, we see that bright quarks carry electroweak and strong charges. Using (2.3-12), we compute:

$$
\begin{array}{lll}
\overleftarrow{q}_{B}^{\mathrm{R}}=\theta_{\mathrm{c}} \overleftarrow{q}_{A}^{\mathrm{R}}-\theta_{s} \overleftarrow{q}_{Z}^{\mathrm{R}}=\frac{1}{6 \theta_{\mathrm{c}}} & \text { and } & \vec{q}_{B}^{\mathrm{R}}=\theta_{\mathrm{c}} \vec{q}_{A}^{\mathrm{R}}-\theta_{s} \vec{q}_{Z}^{\mathrm{R}}=\frac{-1}{3 \theta_{\mathrm{c}}} \\
\grave{q}_{W}^{\mathrm{R}}=\theta_{s} \stackrel{\leftarrow}{q}_{A}^{\mathrm{R}}+\theta_{\mathrm{c}} \grave{q}_{Z}^{\mathrm{R}}=\frac{-1}{2 \theta_{s}} & \text { and } & \vec{q}_{W}^{\mathrm{R}}=\theta_{s} \vec{q}_{A}^{\mathrm{R}}+\theta_{\mathrm{c}} \vec{q}_{Z}^{\mathrm{R}}=0
\end{array}
$$

We thus conclude that:

$$
\begin{gathered}
\overleftarrow{\mathbf{q}}^{C}=\widehat{\mathbf{Z}}\left(\frac{\theta_{s}}{3 \theta_{\mathrm{c}}}-\frac{1}{2 \theta_{\mathrm{c}} \theta_{s}}\right)-\widehat{\mathbf{A}} \frac{1}{3}+\frac{2 \sqrt{2}}{3} \widehat{\boldsymbol{S}}^{\mathrm{c}}=\frac{\widehat{\mathbf{B}}}{6 \theta_{\mathrm{c}}}-\frac{\widehat{\mathbf{W}}}{2 \theta_{\mathrm{s}}}+\frac{2 \sqrt{2}}{3} \widehat{\boldsymbol{S}}^{\mathrm{c}} \\
\overrightarrow{\mathbf{q}}^{C}=\widehat{\mathbf{z}} \frac{\theta_{s}}{3 \theta_{\mathrm{c}}}-\widehat{\mathbf{A}} \frac{1}{3}+\frac{2 \sqrt{2}}{3} \widehat{\boldsymbol{S}}^{\mathrm{c}}=-\frac{\widehat{\mathbf{B}}}{3 \theta_{\mathrm{c}}}+\frac{2 \sqrt{2}}{3} \widehat{\boldsymbol{S}}^{\mathrm{c}}
\end{gathered}
$$




\section{Unified Charge Vectors Theory}

\subsection{Summary}

Let us recap what we have computed in the last four sections. For neutrinos and dark quarks, we have:

$$
\begin{array}{rlrl}
\overleftarrow{\mathrm{q}}^{\nu} & =\frac{1}{2 \theta_{\mathrm{s}} \theta_{\mathrm{c}}} \hat{\mathbf{z}} & ; \overrightarrow{\mathbf{q}}^{v}= & 0 \\
\overleftarrow{\mathrm{q}}^{\mathrm{r}}=\left(\frac{1}{2 \theta_{\mathrm{s}} \theta_{\mathrm{c}}}-\frac{2 \theta_{\mathrm{s}}}{3 \theta_{\mathrm{c}}}\right) \hat{\mathbf{z}}+\frac{2}{3} \widehat{\mathbf{A}}+\frac{2 \sqrt{2}}{3} \widehat{\mathbf{S}}^{\mathrm{r}} ; \overrightarrow{\mathbf{q}}^{\mathrm{r}}=-\frac{2 \theta_{\mathrm{s}}}{3 \theta_{\mathrm{c}}} \hat{\mathbf{z}}+\frac{2}{3} \widehat{\mathbf{A}}+\frac{2 \sqrt{2}}{3} \widehat{\mathbf{S}}^{\mathrm{r}} \\
\overleftarrow{\mathrm{q}}^{\mathrm{g}}=\left(\frac{1}{2 \theta_{\mathrm{s}} \theta_{\mathrm{c}}}-\frac{2 \theta_{\mathrm{s}}}{3 \theta_{\mathrm{c}}}\right) \hat{\mathbf{z}}+\frac{2}{3} \widehat{\mathbf{A}}+\frac{2 \sqrt{2}}{3} \widehat{\mathbf{S}}^{\mathrm{g}} ; \overrightarrow{\mathbf{q}}^{\mathrm{g}}=-\frac{2 \theta_{\mathrm{s}}}{3 \theta_{\mathrm{c}}} \hat{\mathbf{z}}+\frac{2}{3} \widehat{\mathbf{A}}+\frac{2 \sqrt{2}}{3} \widehat{\mathbf{S}}^{\mathrm{g}} \\
\overleftarrow{\mathrm{q}}^{\mathrm{b}}=\left(\frac{1}{2 \theta_{\mathrm{s}} \theta_{\mathrm{c}}}-\frac{2 \theta_{\mathrm{s}}}{3 \theta_{\mathrm{c}}}\right) \hat{\mathbf{z}}+\frac{2}{3} \widehat{\mathbf{A}}+\frac{2 \sqrt{2}}{3} \widehat{\mathbf{S}}^{\mathrm{b}} ; \overrightarrow{\mathbf{q}}^{\mathrm{b}}=-\frac{2 \theta_{\mathrm{s}}}{3 \theta_{\mathrm{c}}} \hat{\mathbf{z}}+\frac{2}{3} \widehat{\mathbf{A}}+\frac{2 \sqrt{2}}{3} \widehat{\mathbf{S}}^{\mathrm{b}}
\end{array}
$$

And for electrons and bright quarks:

$$
\begin{aligned}
& \overleftarrow{\mathrm{q}}^{\mathrm{e}}=\left(\frac{-1}{2 \theta_{\mathrm{s}} \theta_{\mathrm{c}}}+\frac{\theta_{\mathrm{s}}}{\theta_{\mathrm{c}}}\right) \hat{\mathbf{z}}-\widehat{\mathbf{A}} ; \overrightarrow{\mathbf{q}}^{\mathrm{e}}=\frac{\theta_{\mathrm{s}}}{\theta_{\mathrm{c}}} \widehat{\mathbf{z}}-\widehat{\mathbf{A}} \\
& \overleftarrow{\mathrm{q}}^{\mathrm{R}}=\left(\frac{\theta_{s}}{3 \theta_{\mathrm{c}}}-\frac{1}{2 \theta_{\mathrm{s}} \theta_{\mathrm{c}}}\right) \hat{\mathbf{z}}-\frac{1}{3} \widehat{\mathbf{A}}+\frac{2 \sqrt{2}}{3} \widehat{\mathbf{S}}^{\mathrm{r}} ; \overrightarrow{\mathbf{q}}^{\mathrm{R}}=\frac{\theta_{\mathrm{s}}}{3 \theta_{\mathrm{c}}} \widehat{\mathbf{z}}-\frac{1}{3} \widehat{\mathbf{A}}+\frac{2 \sqrt{2}}{3} \widehat{\mathbf{S}}^{\mathrm{r}} \\
& \overleftarrow{\mathrm{q}}^{\mathrm{G}}=\left(\frac{\theta_{s}}{3 \theta_{\mathrm{c}}}-\frac{1}{2 \theta_{\mathrm{s}} \theta_{\mathrm{c}}}\right) \hat{\mathbf{z}}-\frac{1}{3} \widehat{\mathbf{A}}+\frac{2 \sqrt{2}}{3} \widehat{\mathbf{S}}^{\mathrm{g}} ; \overrightarrow{\mathbf{q}}^{\mathrm{G}}=\frac{\theta_{\mathrm{s}}}{3 \theta_{\mathrm{c}}} \widehat{\mathbf{z}}-\frac{1}{3} \widehat{\mathbf{A}}+\frac{2 \sqrt{2}}{3} \widehat{\mathbf{S}}^{\mathrm{g}} \\
& \overleftarrow{\mathrm{q}}^{\mathrm{B}}=\left(\frac{\theta_{s}}{3 \theta_{\mathrm{c}}}-\frac{1}{2 \theta_{\mathrm{s}} \theta_{\mathrm{c}}}\right) \hat{\mathbf{z}}-\frac{1}{3} \widehat{\mathbf{A}}+\frac{2 \sqrt{2}}{3} \widehat{\mathbf{S}}^{\mathrm{b}} ; \overrightarrow{\mathbf{q}}^{\mathrm{B}}=\frac{\theta_{\mathrm{s}}}{3 \theta_{\mathrm{c}}} \widehat{\mathbf{z}}-\frac{1}{3} \widehat{\mathbf{A}}+\frac{2 \sqrt{2}}{3} \widehat{\mathbf{S}}^{\mathrm{b}}
\end{aligned}
$$




\section{Unified Charge Vectors Theory}

Alternatively, using the relations $\widehat{\mathbf{A}}=\theta_{\mathrm{S}} \widehat{\mathbf{W}}+\theta_{c} \widehat{\mathbf{B}}$ and $\widehat{\mathbf{Z}}=\theta_{c} \widehat{\mathbf{W}}-\theta_{\mathbf{S}} \widehat{\mathbf{B}}$, we have for neutrinos and dark quarks:

$$
\begin{aligned}
& \overleftarrow{\mathrm{q}}^{\nu}=\frac{-1}{2 \theta_{\mathrm{c}}} \widehat{\mathbf{B}}+\frac{1}{2 \theta_{\mathrm{s}}} \widehat{\mathbf{W}} \quad ; \overrightarrow{\mathbf{q}}^{v}=0 \\
& \overleftarrow{\mathrm{q}}^{\mathrm{r}}=\frac{1}{6 \theta_{\mathrm{c}}} \widehat{\mathbf{B}}+\frac{1}{2 \theta_{\mathrm{s}}} \widehat{\mathbf{W}}+\frac{2 \sqrt{2}}{3} \widehat{\mathbf{S}}^{\mathrm{r}} ; \overrightarrow{\mathbf{q}}^{\mathrm{r}}=\frac{2}{3 \theta_{\mathrm{c}}} \widehat{\mathbf{B}}+\frac{2 \sqrt{2}}{3} \widehat{\mathbf{S}}^{\mathrm{r}} \\
& \overleftarrow{\mathrm{q}}^{\mathrm{g}}=\frac{1}{6 \theta_{\mathrm{c}}} \widehat{\mathbf{B}}+\frac{1}{2 \theta_{\mathrm{s}}} \widehat{\mathbf{W}}+\frac{2 \sqrt{2}}{3} \widehat{\mathbf{S}}^{\mathrm{g}} ; \overrightarrow{\mathbf{q}}^{\mathrm{g}}=\frac{2}{3 \theta_{\mathrm{c}}} \widehat{\mathbf{B}}+\frac{2 \sqrt{2}}{3} \widehat{\mathbf{S}}^{\mathrm{g}} \\
& \overleftarrow{\mathrm{q}}^{\mathrm{b}}=\frac{1}{6 \theta_{\mathrm{c}}} \widehat{\mathbf{B}}+\frac{1}{2 \theta_{\mathrm{s}}} \widehat{\mathbf{W}}+\frac{2 \sqrt{2}}{3} \widehat{\mathbf{S}}^{\mathrm{b}} ; \overrightarrow{\mathbf{q}}^{\mathrm{b}}=\frac{2}{3 \theta_{\mathrm{c}}} \widehat{\mathbf{B}}+\frac{2 \sqrt{2}}{3} \widehat{\mathbf{S}}^{\mathrm{b}}
\end{aligned}
$$

Likewise, for electrons and bright quarks, we have:

$$
\begin{aligned}
& \overleftarrow{\mathrm{q}}^{\mathrm{e}}=\frac{-1}{2 \theta_{\mathrm{c}}} \widehat{\mathbf{B}}+\frac{-1}{2 \theta_{\mathrm{s}}} \widehat{\mathbf{W}} ; \overrightarrow{\mathbf{q}}^{\mathrm{e}}=\frac{-1}{\theta_{\mathrm{c}}} \widehat{\mathbf{B}} \\
& \overleftarrow{\mathrm{q}}^{\mathrm{R}}=\frac{1}{6 \theta_{\mathrm{c}}} \widehat{\mathbf{B}}+\frac{-1}{2 \theta_{\mathrm{s}}} \widehat{\mathbf{W}}+\frac{2 \sqrt{2}}{3} \widehat{\mathbf{S}}^{\mathrm{r}} ; \overrightarrow{\mathbf{q}}^{\mathrm{R}}=\frac{-1}{3 \theta_{\mathrm{c}}} \widehat{\mathbf{B}}+\frac{2 \sqrt{2}}{3} \widehat{\mathbf{S}}^{\mathrm{r}} \\
& \overleftarrow{\mathrm{q}}^{\mathrm{G}}=\frac{1}{6 \theta_{\mathrm{c}}} \widehat{\mathbf{B}}+\frac{-1}{2 \theta_{\mathrm{s}}} \widehat{\mathbf{W}}+\frac{2 \sqrt{2}}{3} \widehat{\mathbf{S}}^{\mathrm{g}} ; \overrightarrow{\mathbf{q}}^{\mathrm{G}}=\frac{-1}{3 \theta_{\mathrm{c}}} \widehat{\mathbf{B}}+\frac{2 \sqrt{2}}{3} \widehat{\mathbf{S}}^{\mathrm{g}} \\
& \overleftarrow{\mathrm{q}}^{\mathrm{B}}=\frac{1}{6 \theta_{\mathrm{c}}} \widehat{\mathbf{B}}+\frac{-1}{2 \theta_{\mathrm{s}}} \widehat{\mathbf{W}}+\frac{2 \sqrt{2}}{3} \widehat{\mathbf{S}}^{\mathrm{b}} ; \overrightarrow{\mathbf{q}}^{\mathrm{B}}=\frac{-1}{3 \theta_{\mathrm{c}}} \widehat{\mathbf{B}}+\frac{2 \sqrt{2}}{3} \widehat{\mathbf{S}}^{\mathrm{b}}
\end{aligned}
$$




\section{Unified Charge Vectors Theory}

This motivates us to define the Matrix Composition Operator $\circ$ as follows:

$$
\widehat{\mathbf{X}} \circ\left[\begin{array}{llll}
\mathrm{a} & 0 & 0 & 0 \\
0 & \mathbf{b} & 0 & 0 \\
0 & 0 & \mathbf{c} & 0 \\
0 & 0 & 0 & \mathrm{~d}
\end{array}\right] \stackrel{\text { def }}{=}\left[\begin{array}{cccc}
\mathrm{a} \widehat{\mathbf{X}} & 0 & 0 & 0 \\
0 & \mathbf{b} \widehat{\mathbf{X}} & 0 & 0 \\
0 & 0 & \mathbf{c} \widehat{\mathbf{X}} & 0 \\
0 & 0 & 0 & \mathrm{~d} \widehat{\mathbf{X}}
\end{array}\right] \text { as opposed to } \widehat{\mathbf{X}}\left[\begin{array}{cccc}
\mathrm{a} & 0 & 0 & 0 \\
0 & \mathrm{~b} & 0 & 0 \\
0 & 0 & \mathbf{c} & 0 \\
0 & 0 & 0 & \mathrm{~d}
\end{array}\right] \stackrel{\text { def }}{=}\left[\mathrm{a} \widehat{\mathrm{X}}_{0}, \mathrm{~b} \widehat{\mathbf{X}}_{1}, \mathbf{c} \widehat{\mathbf{X}}_{2}, \mathrm{~d} \widehat{\mathbf{X}}_{3}\right]
$$

So, we can now rewrite (2.10-3) and (2.10-4) in matrix form, as follows:

$$
\left[\begin{array}{cccc}
\overleftarrow{\mathrm{q}}^{\boldsymbol{v}} & 0 & 0 & 0 \\
0 & \overleftarrow{\mathrm{q}}^{\mathrm{r}} & 0 & 0 \\
0 & 0 & \overleftarrow{\mathrm{q}}^{\mathrm{g}} & 0 \\
0 & 0 & 0 & \overleftarrow{\mathrm{q}}^{\mathrm{b}}
\end{array}\right]=\frac{\widehat{\mathbf{B}}}{2 \theta_{\mathbf{c}}} \circ\left[\begin{array}{cccc}
-1 & 0 & 0 & 0 \\
0 & \frac{1}{3} & 0 & 0 \\
0 & 0 & \frac{1}{3} & 0 \\
0 & 0 & 0 & \frac{1}{3}
\end{array}\right]+\frac{\widehat{\mathbf{W}}}{2 \theta_{\mathbf{s}}} \circ\left[\begin{array}{cccc}
1 & 0 & 0 & 0 \\
0 & 1 & 0 & 0 \\
0 & 0 & 1 & 0 \\
0 & 0 & 0 & 1
\end{array}\right]+\frac{2 \sqrt{2}}{3}\left[\begin{array}{cccc}
0 & 0 & 0 & 0 \\
0 & \widehat{\mathbf{S}}^{\mathrm{r}} & 0 & 0 \\
0 & 0 & \widehat{\mathbf{S}}^{\mathrm{g}} & 0 \\
0 & 0 & 0 & \hat{\mathbf{S}}^{\mathrm{b}}
\end{array}\right]
$$

And:

$$
\left[\begin{array}{cccc}
\overleftarrow{\mathbf{q}}^{\mathbf{e}} & 0 & 0 & 0 \\
0 & \overleftarrow{\mathbf{q}}^{\mathrm{R}} & 0 & 0 \\
0 & 0 & \overleftarrow{\mathbf{q}}^{\mathrm{G}} & 0 \\
0 & 0 & 0 & \overleftarrow{\mathrm{q}}^{\mathrm{B}}
\end{array}\right]=\frac{\widehat{\mathbf{B}}}{2 \theta_{\mathbf{c}}} \circ\left[\begin{array}{cccc}
-1 & 0 & 0 & 0 \\
0 & \frac{1}{3} & 0 & 0 \\
0 & 0 & \frac{1}{3} & 0 \\
0 & 0 & 0 & \frac{1}{3}
\end{array}\right]-\frac{\widehat{\mathbf{W}}}{2 \theta_{\mathbf{s}}} \circ\left[\begin{array}{cccc}
1 & 0 & 0 & 0 \\
0 & 1 & 0 & 0 \\
0 & 0 & 1 & 0 \\
0 & 0 & 0 & 1
\end{array}\right]+\frac{2 \sqrt{2}}{3}\left[\begin{array}{cccc}
0 & 0 & 0 & 0 \\
0 & \widehat{\mathbf{S}}^{\mathrm{r}} & 0 & 0 \\
0 & 0 & \widehat{\mathbf{S}}^{\mathrm{g}} & 0 \\
0 & 0 & 0 & \widehat{\mathbf{S}}^{\mathrm{b}}
\end{array}\right]
$$

And:

$$
\left[\begin{array}{cccc}
\overrightarrow{\mathbf{q}}^{v} & 0 & 0 & 0 \\
0 & \overrightarrow{\mathbf{q}}^{\mathrm{r}} & 0 & 0 \\
0 & 0 & \overrightarrow{\mathbf{q}}^{\mathrm{g}} & 0 \\
0 & 0 & 0 & \overrightarrow{\mathbf{q}}^{\mathrm{b}}
\end{array}\right]=\frac{\widehat{\mathbf{B}}}{\theta_{\mathrm{c}}} \circ\left[\begin{array}{cccc}
0 & 0 & 0 & 0 \\
0 & \frac{2}{3} & 0 & 0 \\
0 & 0 & \frac{2}{3} & 0 \\
0 & 0 & 0 & \frac{2}{3}
\end{array}\right]+\frac{2 \sqrt{2}}{3}\left[\begin{array}{cccc}
0 & 0 & 0 & 0 \\
0 & \widehat{\mathbf{S}}^{\mathrm{r}} & 0 & 0 \\
0 & 0 & \widehat{\mathbf{S}}^{\mathrm{g}} & 0 \\
0 & 0 & 0 & \widehat{\mathbf{S}}^{\mathrm{b}}
\end{array}\right]
$$

And:

$$
\left[\begin{array}{cccc}
\overrightarrow{\mathbf{q}}^{\mathbf{e}} & 0 & 0 & 0 \\
0 & \overrightarrow{\mathbf{q}}^{\mathrm{R}} & 0 & 0 \\
0 & 0 & \overrightarrow{\mathbf{q}}^{\mathrm{G}} & 0 \\
0 & 0 & 0 & \overrightarrow{\mathbf{q}}^{\mathrm{B}}
\end{array}\right]=-\frac{\widehat{\mathbf{B}}}{\theta_{\mathrm{c}}} \circ\left[\begin{array}{cccc}
1 & 0 & 0 & 0 \\
0 & \frac{1}{3} & 0 & 0 \\
0 & 0 & \frac{1}{3} & 0 \\
0 & 0 & 0 & \frac{1}{3}
\end{array}\right]+\frac{2 \sqrt{2}}{3}\left[\begin{array}{cccc}
0 & 0 & 0 & 0 \\
0 & \widehat{\mathbf{S}}^{\mathrm{r}} & 0 & 0 \\
0 & 0 & \widehat{\boldsymbol{S}}^{\mathrm{g}} & 0 \\
0 & 0 & 0 & \widehat{\mathbf{S}}^{\mathrm{b}}
\end{array}\right]
$$




\section{Unified Charge Vectors Theory}

We can greatly simplify the above equations by defining the following constant matrices:

So:

$$
\mathfrak{T} \stackrel{\text { def }}{=}\left[\begin{array}{cccc}
-1 & 0 & 0 & 0 \\
0 & \frac{1}{3} & 0 & 0 \\
0 & 0 & \frac{1}{3} & 0 \\
0 & 0 & 0 & \frac{1}{3}
\end{array}\right] \quad \text { and } \quad \mathbf{I} \stackrel{\text { def }}{=}\left[\begin{array}{llll}
1 & 0 & 0 & 0 \\
0 & 1 & 0 & 0 \\
0 & 0 & 1 & 0 \\
0 & 0 & 0 & 1
\end{array}\right]
$$

We can now rewrite (2.10-6) and (2.10-7) as follows:

$$
\begin{aligned}
& {\left[\begin{array}{cccc}
\overleftarrow{\mathrm{q}}^{\boldsymbol{v}} & 0 & 0 & 0 \\
0 & \overleftarrow{\mathrm{q}}^{\mathrm{r}} & 0 & 0 \\
0 & 0 & \overleftarrow{\mathrm{q}}^{\mathrm{g}} & 0 \\
0 & 0 & 0 & \overleftarrow{\mathrm{q}}^{\mathrm{b}}
\end{array}\right]=\frac{\widehat{\mathbf{B}}}{2 \theta_{\mathrm{c}}} \circ \mathbf{T}+\frac{\widehat{\mathbf{W}}}{2 \theta_{\mathbf{s}}} \circ \mathbf{I}+\frac{2 \sqrt{2}}{3}\left[\begin{array}{cccc}
0 & 0 & 0 & 0 \\
0 & \widehat{\mathbf{S}}^{\mathrm{r}} & 0 & 0 \\
0 & 0 & \widehat{\mathbf{S}}^{\mathrm{g}} & 0 \\
0 & 0 & 0 & \widehat{\mathbf{S}}^{\mathrm{b}}
\end{array}\right]} \\
& {\left[\begin{array}{cccc}
\overleftarrow{\mathrm{q}}^{\mathrm{e}} & 0 & 0 & 0 \\
0 & \overleftarrow{\mathrm{q}}^{\mathrm{R}} & 0 & 0 \\
0 & 0 & \overleftarrow{\mathbf{q}}^{\mathrm{G}} & 0 \\
0 & 0 & 0 & \overleftarrow{\mathrm{q}}^{\mathrm{B}}
\end{array}\right]=\frac{\widehat{\mathbf{B}}}{2 \theta_{\mathbf{c}}} \circ \mathfrak{T}-\frac{\widehat{\mathbf{W}}}{2 \theta_{\mathbf{s}}} \circ \mathbf{I}+\frac{2 \sqrt{2}}{3}\left[\begin{array}{cccc}
0 & 0 & 0 & 0 \\
0 & \widehat{\mathbf{S}}^{\mathrm{r}} & 0 & 0 \\
0 & 0 & \widehat{\mathbf{S}}^{\mathrm{g}} & 0 \\
0 & 0 & 0 & \widehat{\mathbf{S}}^{\mathrm{b}}
\end{array}\right]}
\end{aligned}
$$

Likewise, (2.10-8) and (2.10-9) become:

$$
\begin{aligned}
& {\left[\begin{array}{cccc}
\overrightarrow{\mathbf{q}}^{\boldsymbol{v}} & 0 & 0 & 0 \\
0 & \overrightarrow{\mathbf{q}}^{\mathrm{r}} & 0 & 0 \\
0 & 0 & \overrightarrow{\mathbf{q}}^{\mathrm{g}} & 0 \\
0 & 0 & 0 & \overrightarrow{\mathbf{q}}^{\mathrm{b}}
\end{array}\right]=\frac{\widehat{\mathbf{B}}}{2 \theta_{\mathrm{c}}} \circ \mathfrak{T}+\frac{\widehat{\mathbf{B}}}{2 \theta_{\mathrm{c}}} \circ \mathbf{I}+\frac{2 \sqrt{2}}{3}\left[\begin{array}{cccc}
0 & 0 & 0 & 0 \\
0 & \widehat{\mathbf{S}}^{\mathrm{r}} & 0 & 0 \\
0 & 0 & \widehat{\mathbf{S}}^{\mathrm{g}} & 0 \\
0 & 0 & 0 & \widehat{\mathbf{S}}^{\mathrm{b}}
\end{array}\right]} \\
& {\left[\begin{array}{cccc}
\overrightarrow{\mathbf{q}}^{\mathrm{e}} & 0 & 0 & 0 \\
0 & \overrightarrow{\mathbf{q}}^{\mathrm{R}} & 0 & 0 \\
0 & 0 & \overrightarrow{\mathbf{q}}^{\mathrm{G}} & 0 \\
0 & 0 & 0 & \overrightarrow{\mathbf{q}}^{\mathrm{B}}
\end{array}\right]=\frac{\widehat{\mathbf{B}}}{2 \theta_{\mathrm{c}}} \circ \mathfrak{T}-\frac{\widehat{\mathbf{B}}}{2 \theta_{\mathrm{c}}} \circ \mathbf{I}+\frac{2 \sqrt{2}}{3}\left[\begin{array}{cccc}
0 & 0 & 0 & 0 \\
0 & \widehat{\mathbf{S}}^{\mathrm{r}} & 0 & 0 \\
0 & 0 & \widehat{\mathbf{S}}^{\mathrm{g}} & 0 \\
0 & 0 & 0 & \widehat{\mathbf{S}}^{\mathrm{b}}
\end{array}\right]}
\end{aligned}
$$

These equations will prove extremely useful when we construct the unified Lagrangian. 


\section{Unified Charge Vectors Theory}

\section{Fermions Interactions}

In this chapter, we investigate fermions interactions.

We begin by considering the classical static potential between two charged particles and derive the concepts of unified-charge-force and potential-bosons.

We then investigate fermions annihilation processes and derive the bosons periodic table. 


\section{Unified Charge Vectors Theory}

\subsection{The Unified Charge Force}

In this section, we want to develop the concept of the unified charge force. Let us begin by considering the equation for the classical electrostatic force between two particles carrying electric charges $q^{a}$ and $q^{b}$. In this case, the force is given by:

$$
\mathrm{F}=-\frac{\partial \mathrm{E}}{\partial \mathrm{r}} \quad \text { where } E=\frac{\alpha}{r} q^{a} q^{b} \quad \text { and } \alpha \stackrel{\text { def }}{=} \frac{\mathrm{e}^{2}}{4 \pi \varepsilon_{0} \hbar c} \quad \text { and } \hbar=c=1
$$

That is to say, the force is obtained by differentiating the potential energy of the two particles, where $r$ is the distance between them.

Next, we promote $q^{a}$ and $q^{b}$ to charge vectors $\overleftrightarrow{\mathbf{q}}^{a}$ and $\overleftrightarrow{\mathbf{q}}^{b}$, and rewrite (3.1-1) as follows:

$$
\mathrm{F}=-\frac{\partial \mathrm{E}}{\partial \mathrm{r}} \quad \text { where } \mathrm{E}=\frac{1}{2}(\overleftarrow{\mathrm{E}}+\overrightarrow{\mathrm{E}}) \text { and } \overleftrightarrow{\mathrm{E}}=\frac{\alpha}{r}\left(\overleftrightarrow{\mathbf{q}}^{a} \cdot \overleftrightarrow{\mathbf{q}}^{b}\right)
$$

This equation suggests a grand unification of all the different forces resulting from the various charge components of $\overleftrightarrow{\mathbf{q}}$. However, (3.1-2) has two issues we need to address. The first has to do with the weak force, and the second with anti-particles.

To understand the problem with the weak force, we first recall that we can express $\overleftrightarrow{\mathbf{q}}$ as follows:

$$
\overleftrightarrow{\mathbf{q}}=\overleftrightarrow{\mathrm{q}}_{z} \widehat{\mathbf{Z}}+\mathrm{q}_{A} \widehat{\mathbf{A}}+\mathrm{q}_{c} \widehat{\mathbf{S}}^{\mathrm{c}} \quad c \in\{\mathrm{r}, \mathrm{g}, \mathrm{b}\}
$$

Substituting this in (3.1-2), and using the orthonormality of $\widehat{\mathbf{Z}}, \widehat{\mathbf{A}}$ and $\widehat{\mathbf{S}}^{\mathrm{c}}$, we obtain:

$$
\overleftrightarrow{\mathrm{E}}=\frac{\alpha}{r}\left(\overleftrightarrow{\mathbf{q}}^{a} \cdot \overleftrightarrow{\mathbf{q}}^{b}\right)=\frac{\alpha}{r}\left(\overleftrightarrow{\mathrm{q}}_{z}^{a} \overleftrightarrow{\mathrm{q}}_{z}^{b}\right)+\frac{\alpha}{r}\left(\mathrm{q}_{A}^{a} \mathrm{q}_{A}^{b}\right)+\frac{\alpha}{r}\left(\mathrm{q}_{c}^{a} \mathrm{q}_{c}^{b}\right)
$$

We can see that due to the orthogonality of $\widehat{\mathbf{Z}}, \widehat{\mathbf{A}}$ and $\widehat{\mathbf{S}}^{\mathrm{c}}$, each of the charge components of $\overleftrightarrow{\mathbf{q}}^{a}$ interacts separately with the corresponding component in $\overleftrightarrow{\mathbf{q}}^{b}$ - as indeed they should.

The problem is that the weak force generated by the weak charges $\overleftrightarrow{\mathrm{q}}_{z}^{a}$ and $\overleftrightarrow{\mathrm{q}}_{z}^{b}$ is supposed to decay exponentially. That is to say; we should have:

$$
\overleftrightarrow{\mathrm{E}}_{Z}=\frac{e^{-m_{z} r}}{r} \alpha\left(\overleftrightarrow{\mathrm{q}}_{z}^{a} \overleftrightarrow{\mathrm{q}}_{z}^{b}\right) \text { rather than } \overleftrightarrow{\mathrm{E}}_{Z}=\frac{\alpha}{r}\left(\overleftrightarrow{\mathrm{q}}_{z}^{a} \overleftrightarrow{\mathrm{q}}_{z}^{b}\right)
$$

Where $m_{z}$ is some positive constant that ensures the decay of the weak force. This suggests that we reformulate (3.1-4) as follows:

$$
\overleftrightarrow{\mathrm{E}}=\frac{\alpha}{r}\left(\overleftrightarrow{\mathbf{q}}^{a} \cdot \overleftrightarrow{\mathbf{q}}^{b}+\left(e^{-m_{z} r}-1\right)\left(\widehat{\mathbf{Z}} \cdot \overleftrightarrow{\mathbf{q}}^{a}\right)\left(\widehat{\mathbf{Z}} \cdot \overleftrightarrow{\mathbf{q}}^{b}\right)\right)
$$




\section{Unified Charge Vectors Theory}

To convince ourselves that the issue is resolved, we recall that $\overleftrightarrow{\mathrm{q}}_{z}=\widehat{\mathbf{Z}} \cdot \overleftrightarrow{\mathbf{q}}$, and write:

$$
\begin{gathered}
\overleftrightarrow{\mathrm{E}}=\frac{\alpha}{r}\left(\left(\overleftrightarrow{\mathrm{q}}_{z}^{a} \overleftrightarrow{\mathrm{q}}_{z}^{b}\right)+\left(\mathrm{q}_{A}^{a} \mathrm{q}_{A}^{b}\right)+\left(\mathrm{q}_{c}^{a} \mathrm{q}_{c}^{b}\right)+\left(e^{-m_{z}^{2} r}-1\right)\left(\overleftrightarrow{\mathrm{q}}_{z}^{a} \overleftrightarrow{\mathrm{q}}_{z}^{b}\right)\right) \\
=\frac{\alpha}{r}\left(e^{-m_{z} r}\left(\overleftrightarrow{\mathrm{q}}_{z}^{a} \overleftrightarrow{\mathrm{q}}_{z}^{b}\right)+\left(\mathrm{q}_{A}^{a} \mathrm{q}_{A}^{b}\right)+\left(\mathrm{q}_{c}^{a} \mathrm{q}_{c}^{b}\right)\right)
\end{gathered}
$$

So, all is well now. Notice that for small enough distances, we have $e^{-m_{z} r}-1 \approx 0$, and we thus recover back (3.1-4).

As an example, let us consider the classical unified force between dark red and green quarks. We recall that by (2.8-9) and (2.8-10), the unified charge vectors of dark quarks are given by:

$$
\overleftarrow{\mathrm{q}}^{c}=\widehat{\mathbf{Z}}\left(\frac{1}{2 \theta_{\mathrm{c}} \theta_{s}}-\frac{2 \theta_{s}}{3 \theta_{\mathrm{c}}}\right)+\widehat{\mathbf{A}} \frac{2}{3}+\frac{2 \sqrt{2}}{3} \widehat{\mathbf{S}}^{\mathrm{c}} \text { and } \overrightarrow{\mathbf{q}}^{c}=-\widehat{\mathbf{Z}} \frac{2 \theta_{s}}{3 \theta_{\mathrm{c}}}+\widehat{\mathbf{A}} \frac{2}{3}+\frac{2 \sqrt{2}}{3} \widehat{\mathbf{S}}^{\mathrm{c}}
$$

We also recall that by (2.4-5), we have $\widehat{\mathbf{S}}^{\mathrm{r}} \cdot \widehat{\mathbf{S}}^{\mathrm{g}}=-\frac{1}{2}$. Plugging this in equation (3.1-7), we obtain the following potential energies between the two quarks:

$$
\begin{gathered}
\overleftarrow{\mathrm{E}}=\frac{\alpha}{r}\left(e^{-m_{z} r}\left(\frac{1}{2 \theta_{\mathrm{c}} \theta_{s}}-\frac{2 \theta_{s}}{3 \theta_{\mathrm{c}}}\right)^{2}+\left(\frac{2}{3}\right)^{2}-\frac{1}{2}\left(\frac{2 \sqrt{2}}{3}\right)^{2}\right) \\
\overrightarrow{\mathrm{E}}=\frac{\alpha}{r}\left(e^{-m_{z} r}\left(\frac{2 \theta_{s}}{3 \theta_{\mathrm{c}}}\right)^{2}+\left(\frac{2}{3}\right)^{2}-\frac{1}{2}\left(\frac{2 \sqrt{2}}{3}\right)^{2}\right)
\end{gathered}
$$

Hence, the total unified potential energy is given by:

$$
\mathrm{E} \stackrel{\text { def }}{=} \frac{1}{2}(\overleftarrow{\mathrm{E}}+\overrightarrow{\mathrm{E}})=\frac{\alpha}{r}(\underbrace{\frac{e^{-m_{z} r}}{2}\left(\left(\frac{1}{2 \theta_{\mathrm{c}} \theta_{s}}-\frac{2 \theta_{s}}{3 \theta_{\mathrm{c}}}\right)^{2}+\left(\frac{2 \theta_{s}}{3 \theta_{\mathrm{c}}}\right)^{2}\right)+\left(\frac{2}{3}\right)^{2}}_{\text {Electroweak Repulsion }}-\underbrace{\left.-\frac{1}{2}\left(\frac{2 \sqrt{2}}{3}\right)^{2}\right)}_{\text {Strong Attraction }}
$$

We, therefore, see that red and green quarks repel each other via the weak and electric forces but attract each other via the strong force. Notice that this attraction is a direct consequence of the relation $\hat{\mathbf{S}}^{\mathrm{r}} \cdot \hat{\mathbf{S}}^{\mathrm{g}}=-\frac{1}{2}$, which results from the fact that the three strong charge direction $\widehat{\mathbf{S}}^{\mathrm{r}}, \hat{\mathbf{S}}^{\mathrm{g}}$, and $\widehat{\mathbf{S}}^{\mathrm{b}}$ share a 2-dimensional charge plane, and hence have a negative projection on each other, as can be seen in diagram (2.4-1).

We also notice that the strong attraction between two quarks of different colors has half the strength of the repulsion strength between two quarks of the same color. This result is in perfect agreement with QCD. 


\section{Unified Charge Vectors Theory}

It is also interesting to note that the strong attraction between two dark quarks is not strong at all. In fact, it precisely balances the electric repulsion between them. Indeed, by (3.1-11) we see that the total electric and strong potential between two dark quarks of different colors is given by:

$$
\mathrm{E}_{A}+\mathrm{E}_{S}=\frac{\alpha}{r}\left(\left(\frac{2}{3}\right)^{2}-\frac{1}{2}\left(\frac{2 \sqrt{2}}{3}\right)^{2}\right)=\frac{\alpha}{r}\left(\frac{4}{9}-\frac{4}{9}\right)=0
$$

Hence, apart from weak repulsion, dark quarks do not exert any other net force on each other. This is also in perfect agreement with QCD asymptotic freedom, according to which the strong force becomes strong due to quantum screening effects, and not due to a large classical coupling constant.

Next, we consider the classical unified charge force between a particle and an anti-particle. We recall that by (2.5-3) and (2.5-4), we have:

$$
\overleftarrow{\overline{\mathrm{q}}}=-\overrightarrow{\mathrm{q}} \text { and } \overrightarrow{\mathrm{q}}=-\overleftarrow{\mathrm{q}}
$$

Hence, in this case (3.1-6) should have the form:

And:

$$
\overleftarrow{\mathrm{E}}=\frac{\alpha}{r}\left(\overleftarrow{\mathrm{q}}^{a} \cdot \overrightarrow{\overline{\mathbf{q}}}^{b}+\left(e^{-m_{z}^{2} r}-1\right)\left(\hat{\mathbf{Z}} \cdot \overleftarrow{\mathrm{q}}^{a}\right)\left(\hat{\mathbf{Z}} \cdot \overrightarrow{\overline{\mathbf{q}}}^{b}\right)\right)
$$

$$
\overrightarrow{\mathrm{E}}=\frac{\alpha}{r}\left(\overrightarrow{\mathbf{q}}^{a} \cdot \overleftarrow{\mathrm{q}}^{b}+\left(e^{-m_{z}^{2} r}-1\right)\left(\hat{\mathbf{z}} \cdot \overrightarrow{\mathbf{q}}^{a}\right)\left(\hat{\mathbf{z}} \cdot \overleftarrow{\mathrm{q}}^{b}\right)\right)
$$

That is to say, the directions in (3.1-6) should be reversed for anti-particles. 


\section{Unified Charge Vectors Theory}

\subsection{Potential Bosons}

Recall that in (3.1-7) we had:

We also have:

$$
\overleftrightarrow{\mathrm{E}}=\frac{\alpha}{r}\left(e^{-m_{z} r}\left(\stackrel{\mathrm{q}}{\mathrm{q}}_{z}^{a} \overleftrightarrow{\mathrm{q}}_{z}^{b}\right)+\left(\mathrm{q}_{A}^{a} \mathrm{q}_{A}^{b}\right)+\left(\mathrm{q}_{c}^{a} \mathrm{q}_{c}^{b}\right)\right)
$$

$$
\overleftrightarrow{\mathrm{q}}_{z}=\widehat{\mathbf{Z}} \cdot \overleftrightarrow{\mathbf{q}} ; \overleftrightarrow{\mathrm{q}}_{A}=\widehat{\mathbf{A}} \cdot \overleftrightarrow{\mathbf{q}} ; \overleftrightarrow{\mathrm{q}}_{c}=\widehat{\mathbf{S}}_{c} \cdot \overleftrightarrow{\mathbf{q}}
$$

Plugging (3.2-2) in (3.2-1), we obtain:

Where:

$$
\overleftrightarrow{\mathrm{E}}=\overleftrightarrow{\mathrm{E}}_{Z}+\overleftrightarrow{\mathrm{E}}_{A}+\overleftrightarrow{\mathrm{E}}_{S}
$$

$$
\begin{aligned}
& \overleftrightarrow{\mathrm{E}}_{Z}=\frac{e^{-m_{z} r}}{r}\left(\sqrt{\alpha} \hat{\mathbf{Z}} \cdot \overleftrightarrow{\mathbf{q}}^{a}\right)\left(\sqrt{\alpha} \widehat{\mathbf{Z}} \cdot \overleftrightarrow{\mathbf{q}}^{b}\right) \\
& \overleftrightarrow{\mathrm{E}}_{A}=\frac{1}{r}\left(\sqrt{\alpha} \widehat{\mathbf{A}} \cdot \overleftrightarrow{\mathbf{q}}^{a}\right)\left(\sqrt{\alpha} \widehat{\mathbf{A}} \cdot \overleftrightarrow{\mathbf{q}}^{b}\right) \\
& \overleftrightarrow{\mathrm{E}}_{S}=\frac{1}{r}\left(\sqrt{\alpha} \widehat{\mathbf{S}}_{c} \cdot \overleftrightarrow{\mathbf{q}}^{a}\right)\left(\sqrt{\alpha} \widehat{\mathbf{S}}_{c} \cdot \overleftrightarrow{\mathbf{q}}^{b}\right)
\end{aligned}
$$

We can associate the following three diagrams to the above three potential energy interactions:
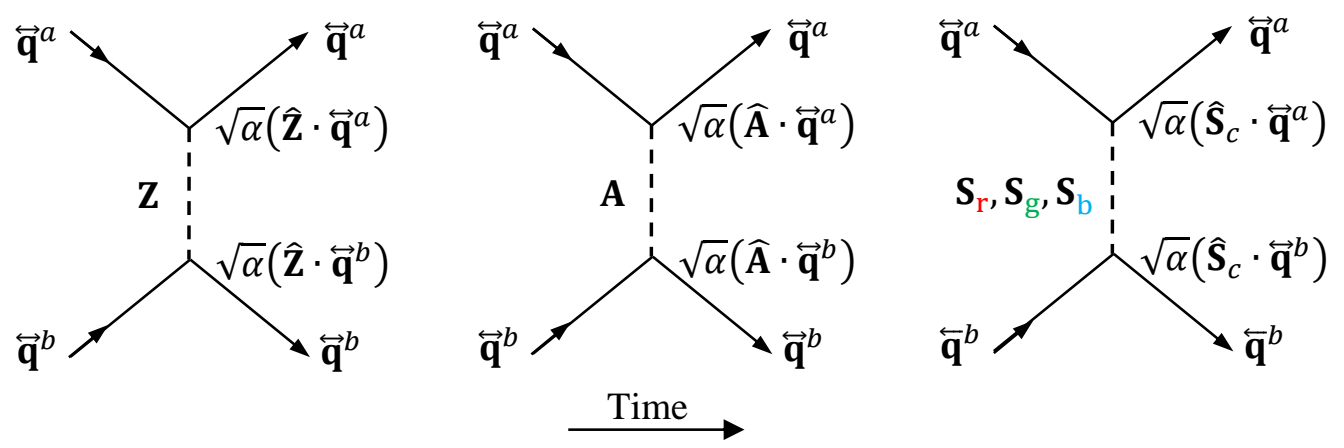

Fermions interacting by "exchanging” $\boldsymbol{Z}, \boldsymbol{A}$ and $\boldsymbol{S}_{c}$ potential bosons

These diagrams describe the interaction between two fermions (via their potential energy) as an exchange of "potential-particles", to which we shall refer as Potential Bosons (i.e., bosons which mediate the potential energy between particles). We denote these potential bosons by $\mathbf{Z}, \mathbf{A}, \mathbf{S}_{\mathrm{r}}, \mathbf{S}_{\mathrm{g}}, \mathbf{S}_{\mathrm{b}}$.

Hence, to get the left and right interaction strengths between, say, the electromagnetic-potential mediator (better known as the Photon), and any fermion with charge vectors $\overleftrightarrow{\mathbf{q}}$, we simply have to compute $\sqrt{\alpha}(\widehat{\mathbf{A}} \cdot \overleftrightarrow{\mathbf{q}})$. 


\section{Unified Charge Vectors Theory}

\subsection{Fermions Annihilation}

Quantum Field Theory teaches us that the existence of fermions interactions diagrams (3.2-7) implies the existence of corresponding Fermions Annihilation \& Creation Diagrams, which can be obtained by rotating the interaction diagrams by $90^{\circ}$ counterclockwise, as follows:
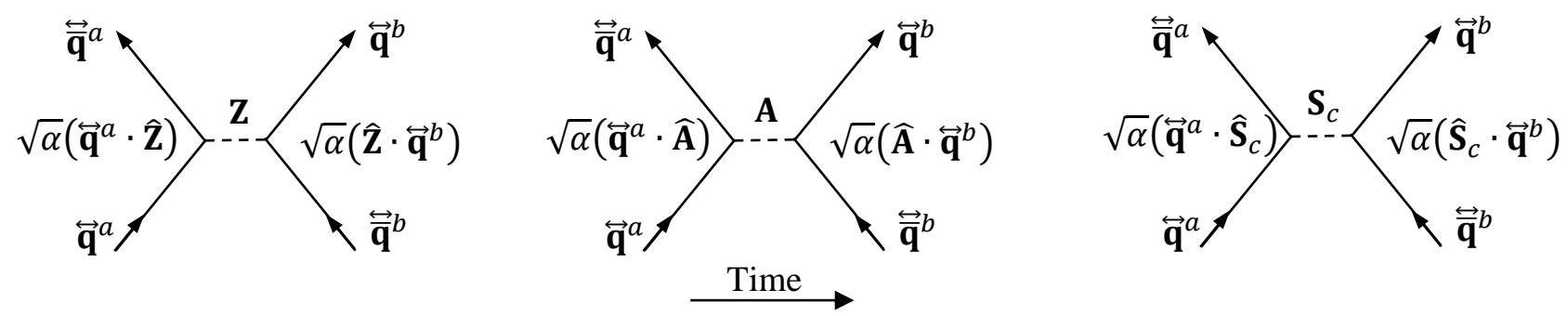

Fermions annihilation \& creation diagrams

These diagrams describe the annihilation of a fermion/anti-fermion pair with charge vectors $\overleftrightarrow{\mathbf{q}}^{a}$ and $\overleftrightarrow{\mathbf{q}}^{a}$ into any of the different potential bosons particles $Z, A, \mathbf{S}_{r}, \mathbf{S}_{\mathrm{g}}, \mathbf{S}_{\mathrm{b}}$. These bosons may then decay into a new pair of

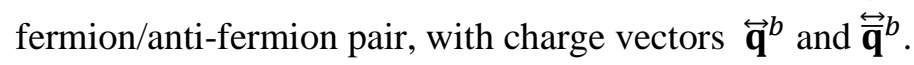

Diagrams (3.3-1) imply that we can also refer to the potential bosons as Gateway Bosons, whereby that we mean - bosons that can "take us" from one pair of fermions to a different pair. This immediately raises the question regarding the possible existence of additional such gateway bosons which can take any fermion/antifermion pair from different breeds and turn them into some other pair of fermion/anti-fermion, also from different breeds. The following diagram depicts such general crossbreed reactions:

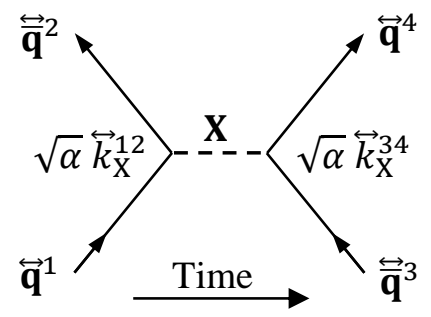

Crossbreed fermions annihilation \& creation

Where $\sqrt{\alpha} \overleftarrow{k}_{\mathrm{X}}^{12}, \sqrt{\alpha} \vec{k}_{\mathrm{X}}^{12}, \sqrt{\alpha} \overleftarrow{k}_{\mathrm{X}}^{34}$, and $\sqrt{\alpha} \vec{k}_{\mathrm{X}}^{34}$ are, respectively, the left and right coupling coefficients that are associated with these kinds of processes.

Since we now allow for the $\left(\mathbf{q}^{1}, \overline{\mathbf{q}}^{2}\right)$ pair to be of different breeds, we refer to such processes as Crossbreed Annihilations. The same, of course, goes for the outgoing pair $\left(\mathbf{q}^{4}, \overline{\mathbf{q}}^{3}\right)$. 


\section{Unified Charge Vectors Theory}

To guide us in the discovery of these possible crossbreed annihilation processes, we rely on a simple quantum mechanical principle, according to which - "Anything that can happen, will indeed happen", where, in our case, "can happen" means:

Annihilation processes must involve a particle and an anti-particle.

Annihilation processes must respect the unified charge conservation law.

Restriction (3.3-3) eliminates annihilation processes that do not involve a fermion/anti-fermion pair, as depicted below:

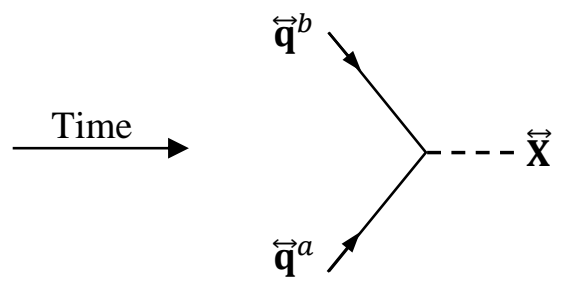

Forbidden fermions annihilations

This prevents the catastrophe of proton decay, proposed by various grand unification theories, which occur if we allow bright-quarks to annihilate dark-quarks (rather than anti-dark-quarks). Hence, restriction (3.3-3) keeps the protons safe and sound for the simple reason that protons do not contain anti-quarks.

Next, we consider restriction (3.3-4), which ensures charge conservation. To achieve that, we demand:

$$
\overleftrightarrow{\mathbf{q}}^{1}-\overleftrightarrow{\mathbf{q}}^{2}=\overleftrightarrow{\mathbf{q}}^{4}-\overleftrightarrow{\mathbf{q}}^{3}
$$

That is to say, the total charge vectors of the incoming particles must equal the total charge vectors of the outgoing particles. We shall refer to (3.3-6) as the Unified Charge Conservation Law. 


\section{Unified Charge Vectors Theory}

We are now ready to discover all possible annihilation processes by using (2.10-3) and (2.10-4) and finding all the double pairs of particles and anti-particles which respect the unified charge conservation law (3.3-6). The result of this tedious work gives us the following Bosons Periodic Table.

\begin{tabular}{|c|c|c|c|c|c|c|c|c|c|}
\hline & $\mathrm{c} 1$ & c2 & c3 & $\mathrm{c} 4$ & $\mathrm{c} 5$ & $\mathrm{c} 6$ & $\mathrm{c} 7$ & $\mathrm{c} 8$ \\
\hline & & $\overleftrightarrow{\mathbf{q}}^{v}$ & $\overleftrightarrow{\mathbf{q}}^{\mathrm{r}}$ & $\overleftrightarrow{\mathbf{q}}^{\mathrm{g}}$ & $\overleftrightarrow{\mathbf{q}}^{\mathrm{b}}$ & $\overleftrightarrow{\mathrm{q}^{\mathrm{e}}}$ & $\stackrel{\leftrightarrows}{\mathrm{q}} \mathrm{R}$ & $\stackrel{\overleftrightarrow{\mathbf{q}} G}{\mathrm{G}}$ & 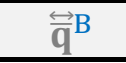 \\
\hline r1 & $\overleftrightarrow{\mathbf{q}}^{v}$ & $\mathbf{Z}$ & $\mathbf{W}^{+\overline{\mathrm{r}}}$ & $\mathbf{W}^{+\bar{g}}$ & $\mathbf{w}^{+\bar{b}}$ & $\mathbf{W}^{+}$ & $\mathrm{W}^{+\overline{\mathrm{r}} \boldsymbol{v}}$ & $\mathrm{W}^{+\overline{\mathrm{g}} v}$ & $\mathrm{~W}^{+\overline{\mathrm{b}} v}$ \\
\hline r2 & $\overleftrightarrow{\mathbf{q}}^{\mathrm{r}}$ & $\mathbf{w}^{-\mathrm{r}}$ & $\mathbf{A}, \mathbf{Z}, \mathbf{S}^{\mathrm{r}}$ & $\mathbf{G}^{\overline{\mathrm{gr}}}$ & $\mathbf{G}^{\overline{\mathrm{b}} \mathrm{r}}$ & $W^{+\bar{e} r}$ & $\mathbf{W}^{+}$ & $W^{+\bar{g} r}$ & $\mathbf{W}^{+\overline{\mathrm{b}} \mathrm{r}}$ \\
\hline r3 & $\overleftrightarrow{\mathbf{q}}^{\mathrm{g}}$ & $\mathbf{W}^{-g}$ & $\mathbf{G}^{\overline{\mathrm{r}} \mathrm{g}}$ & $\mathrm{A}, \mathrm{Z}, \mathbf{S}^{\mathrm{g}}$ & $\mathbf{G}^{\overline{\mathrm{b}} \mathrm{g}}$ & $\mathbf{W}^{+\bar{e} g}$ & $\mathbf{W}^{+\overline{\mathbf{r}} \mathrm{g}}$ & $\overleftrightarrow{\mathbf{W}}^{+}$ & $\mathbf{W}^{+\bar{b} g}$ \\
\hline r4 & $\overleftrightarrow{\mathbf{q}}^{\mathrm{b}}$ & $\mathbf{w}^{-\mathrm{b}}$ & $\mathbf{G}^{\overline{\mathrm{rb}}}$ & $\mathbf{G}^{\overline{\mathrm{g} b}}$ & $\mathbf{A}, \mathbf{Z}, \mathbf{S}^{\mathrm{b}}$ & $\mathrm{W}^{+\overline{\mathrm{e}} \mathrm{b}}$ & $\mathrm{W}^{+\mathrm{r} \mathrm{b}}$ & $\mathrm{W}^{+\bar{g} \mathrm{~b}}$ & $\mathbf{w}^{+}$ \\
\hline r5 & $\overleftrightarrow{\mathrm{q}}^{\mathrm{e}}$ & $\mathbf{W}^{-}$ & $W^{-\bar{r} e}$ & $\mathrm{~W}^{-\overline{\mathrm{g}} \mathrm{e}}$ & $W^{-\bar{b} e}$ & $A, Z$ & $\mathbf{W}^{+\bar{r}}$ & $\mathbf{W}^{+\bar{g}}$ & $\mathbf{W}^{+\bar{b}}$ \\
\hline r6 & $\overleftrightarrow{\mathrm{q}}^{\mathrm{R}}$ & $\mathrm{W}^{-\bar{v} \mathrm{r}}$ & $\mathbf{W}^{-}$ & $\mathrm{W}^{-\overline{\mathrm{g}} \mathrm{r}}$ & $W^{-\bar{b} r}$ & $\mathbf{W}^{-\mathrm{r}}$ & $\mathbf{A}, \mathbf{Z}, \mathbf{S}^{\mathrm{r}}$ & $\mathbf{G}^{\bar{g} \mathrm{r}}$ & $\mathbf{G}^{\bar{b} r}$ \\
\hline r7 & $\overleftrightarrow{\mathbf{q}}^{\mathrm{G}}$ & $W^{-\bar{v} g}$ & $\mathbf{W}^{-\overline{\mathrm{r}} \mathrm{g}}$ & $\mathbf{W}^{-}$ & $W^{-\bar{b} g}$ & $\mathbf{W}^{-\mathrm{g}}$ & $\mathbf{G}^{\overline{\mathrm{r} g}}$ & $\mathbf{A}, \mathbf{Z}, \mathbf{S}^{\mathrm{g}}$ & $\mathbf{G}^{\overline{\mathrm{b}} \mathrm{g}}$ \\
\hline r8 & $\overleftrightarrow{\mathrm{q}}^{\mathrm{B}}$ & $\mathrm{W}^{-\bar{v} \mathrm{~b}}$ & $\mathrm{~W}^{-\overline{\mathrm{r} b}}$ & $\mathbf{W}^{-\overline{\mathrm{g}} \mathrm{b}}$ & $\mathbf{w}^{-}$ & $\mathbf{W}^{-b}$ & $\mathbf{G}^{\overline{\mathrm{r} b}}$ & $\mathbf{G}^{\overline{\mathrm{g} b}}$ & $\mathbf{A}, \mathbf{Z}, \mathbf{S}^{\mathrm{b}}$ \\
\hline
\end{tabular}

The bosons periodic table

The structure of the bosons periodic table is rather simple. At the leftmost column (referred to as column \#0), we have the list of all fermions-types, where the first four are the dark types (i.e., neutrinos and dark quarks), and the last four are the bright types (i.e., electrons and bright quarks). Likewise, the top row (referred to as row \#0) lists the anti-types in the same order.

Each table cell defines the boson generated by the annihilation of the two corresponding fermions in its row and column. So, for instance, the boson located in $(\mathrm{r} 5, \mathrm{c} 1)$ is denoted by $\mathbf{W}^{-}$. The fermions appearing at the leftmost entry of row 5 are the electrons $\overleftrightarrow{\mathrm{q}}^{\mathrm{e}}$, and the anti-fermions at the top of column 1 are the anti-neutrinos $\overleftrightarrow{\mathbf{q}}^{v}$. Hence, this cell describes the annihilation of an electron by an anti-neutrino producing a $\mathbf{W}^{-}$boson with total charge vectors $\overleftrightarrow{\mathbf{q}}^{e}-\overleftrightarrow{\mathbf{q}}^{v}$, as depicted in the following diagram:

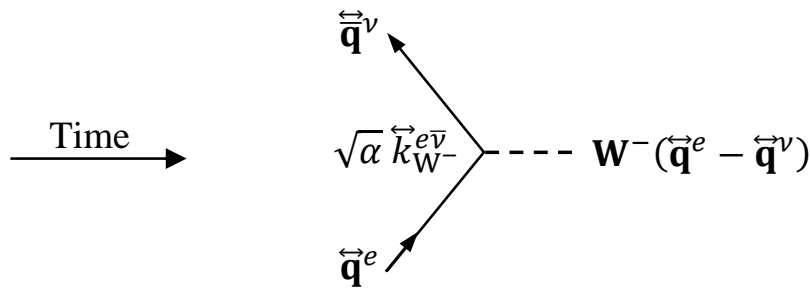

Electron/Anti-neutrino annihilation 


\section{Unified Charge Vectors Theory}

Along the diagonal of the bosons periodic table, we recognize the potential bosons responsible for the annihilation processes we have described in diagram (3.3-1). Due to the bosons periodic table structure, the bosons located above the diagonal are the anti-particles of the bosons situated below it.

To understand in what sense is this table periodic, we notice that all the bosons marked by black can be found in more than a single cell of the table, while those marked by gray occupy only a single cell. Hence, some bosons repeat themselves, while others don't. We refer to repeating bosons as Gateway Bosons, while nonrepeating bosons are called Dead-end Bosons. Notice that the potential bosons which appear along the diagonal are all gateway since each of them appears in more than one cell in the table.

To understand the difference between gateway and dead-end bosons, we refer back to diagram (3.3-2). As discussed, this diagram describes the process of annihilation of the fermion $\overleftrightarrow{\mathbf{q}}^{1}$ by an anti-fermion $\overleftrightarrow{\mathbf{q}}^{2}$ into some boson $\mathbf{X}$, which then decays to a fermion $\overleftrightarrow{\mathbf{q}}^{4}$ and an anti-fermion $\stackrel{\leftrightarrow}{\mathbf{q}}^{3}$.

Now, the bosons periodic table tells us that in order for both of these reactions to occur (i.e., annihilation followed by creation), the $\mathbf{X}$ boson must appear both in the cell corresponding to $\left(\overleftrightarrow{\mathbf{q}}^{1}, \overleftrightarrow{\mathbf{q}}^{2}\right)$, as well as in the cell corresponding to $\left(\overleftrightarrow{\mathbf{q}}^{4}, \overleftrightarrow{\mathbf{q}}^{3}\right)$.

Hence, if $\mathbf{X}$ is a dead-end boson, then by definition, it appears in only one of these cells, which leads us to conclude that $\overleftrightarrow{\mathbf{q}}^{4}=\overleftrightarrow{\mathbf{q}}^{1}$ and $\overleftrightarrow{\mathbf{q}}^{3}=\overleftrightarrow{\mathbf{q}}^{2}$. That is to say; dead-end bosons have nowhere to go but to disintegrate back to exactly the same fermions which created them in the first place. Hence their name dead-end, meaning leading nowhere.

On the other hand, if $\mathbf{X}$ is a gateway boson, then by definition, it appears in more than a single cell in the table, and hence we may have $\overleftrightarrow{\mathbf{q}}^{4} \neq \overleftrightarrow{\mathbf{q}}^{1}$ and/or $\overleftrightarrow{\mathbf{q}}^{3} \neq \overleftrightarrow{\mathbf{q}}^{2}$. That is to say; gateway bosons can take us from one pair of fermions to a different pair. Hence their name Gateway, meaning bosons that connect two different pairs (or cells) in the bosons periodic table.

Notice that if we set $\overleftrightarrow{\mathbf{q}}^{1}=\overleftrightarrow{\mathbf{q}}^{2}$ and $\overleftrightarrow{\mathbf{q}}^{3}=\overleftrightarrow{\mathbf{q}}^{4}$, we recover back the special case of inner-breed annihilation depicted in (3.3-1), and $\mathbf{X}$ then becomes one of the potential bosons appearing in the bosons periodic table diagonal.

Though dead-end bosons can only disintegrate back to the fermions which created them, they do play a role in particle interactions. To see why, we rotate diagram (3.3-2) $90^{\circ}$ clockwise and obtain the following Crossbreed Interaction Diagram:

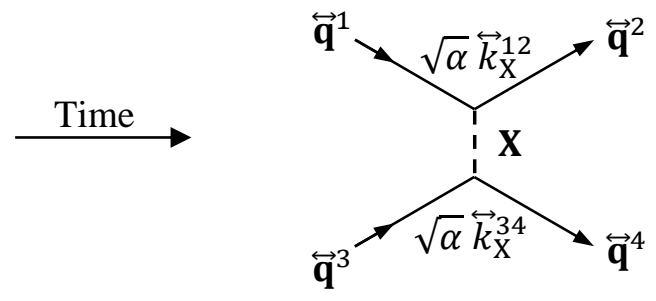

Crossbreed fermions interactions 


\section{Unified Charge Vectors Theory}

Diagram (3.3-9) describes the process of $\overleftrightarrow{\mathbf{q}}^{1}$ decaying into $\overleftrightarrow{\mathbf{q}}^{2}$ and $\mathbf{X}$, which then reacts with $\overleftrightarrow{\mathbf{q}}^{3}$ to form $\overleftrightarrow{\mathbf{q}}^{4}$. Or, equivalently, $\overleftrightarrow{\mathbf{q}}^{3}$ decaying into $\overleftrightarrow{\mathbf{q}}^{4}$ and $\mathbf{X}$, which then reacts with $\overleftrightarrow{\mathbf{q}}^{1}$ to form $\overleftrightarrow{\mathbf{q}}^{2}$. Now, we have already seen that if $\mathbf{X}$ is a dead-end boson, then we must have $\overleftrightarrow{\mathbf{q}}^{4}=\overleftrightarrow{\mathbf{q}}^{1}$ and $\overleftrightarrow{\mathbf{q}}^{3}=\overleftrightarrow{\mathbf{q}}^{2}$, which gives us this:

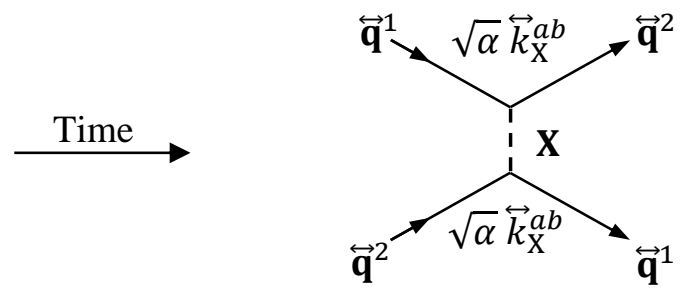

Crossbreed dead-end bosons interactions

Comparing (3.3-10) to (3.2-7), we see that dead-end bosons provide yet another channel through which two fermions can interact.

Finally, we want to introduce the terminology associated with the various bosons appearing in the bosons periodic table (3.3-7).

We are already familiar with the diagonal bosons, i.e., $\mathbf{A}, \mathbf{Z}, \mathbf{S}^{\mathrm{r}}, \mathbf{S}^{\mathrm{g}}, \mathbf{S}^{\mathrm{b}}$, which we have also referred to as potential bosons. Since each of these bosons appears in more than a single cell, we conclude that they are all gateway bosons.

The various $\mathbf{G}^{\overline{\mathrm{x}} \mathrm{y}}$ bosons appearing both in the upper-left and lower-right quarters are collectively referred to as Gateway Gluons. Since they appear in both quarters, they are gateway. Their positions in the table imply they interact with quarks only.

The three bosons $\mathbf{W}^{+\bar{r}}, \mathbf{W}^{+\bar{g}}, \mathbf{W}^{+\bar{b}}$ (and their anti-particles $\mathbf{W}^{-\mathrm{r}}, \mathbf{W}^{-\mathrm{g}}, \mathbf{W}^{-\mathrm{b}}$ ) also appear both in the upper-left and the lower-right quarters and are therefore also gateway. These are called Gateway Leptoquarks since they interact both with quarks and leptons (i.e., neutrinos and electrons).

Similarly, the bosons $\mathbf{W}^{+\bar{r} v}, W^{+\bar{g} v}, W^{+\bar{b} v}, W^{+\bar{e} r}, W^{+\bar{e} g}, W^{+\bar{e} b}$ (and their anti-particles $W^{-\bar{v} r}, W^{-\bar{v} g}, W^{+\bar{v} b}$, $\left.\mathrm{W}^{-\overline{\mathrm{r}} \mathrm{e}}, \mathrm{W}^{-\overline{\mathrm{g} e}}, \mathrm{~W}^{-\overline{\mathrm{b}} \mathrm{e}}\right)$, are called Dead-End Leptoquarks.

Moving on to the upper-right and lower-left diagonals, we identify there the $\mathbf{W}^{+}$boson (and its anti-particle $\mathbf{W}^{-}$). Again, since each of these bosons populates four different cells, they are clearly gateway. As an example involving these bosons, we can mention the familiar beta decay facilitated by the $\mathbf{W}^{-}$.

Finally, we have the dead-end bosons $W^{+\bar{g} r}, W^{+\bar{b} r}, W^{+\bar{b} g}, W^{+\bar{r} g}, W^{+\bar{r} b}, W^{+\bar{g} b}$ located in the upper-right quarter, and their corresponding anti-particles in the lower-left quarter. We referred collectively to these bosons as Dead-End Weak Gluons. Like gateway gluons, their positions in the table imply they interact with quarks only. 


\section{Unified Charge Vectors Theory}

To understand why leptoquarks have escaped detection so far, we consider the following leptoquark/antileptoquark pair production processes resulting from colliding protons. For the dead-end red leptoquarks, we have:

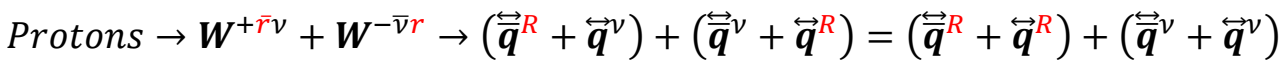

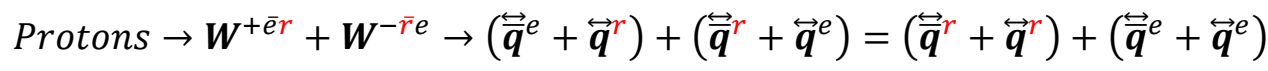

Both of these cases simply result in a stream of fermion/anti-fermion pairs. However, such fermions pairs can be generated without leptoquarks and thus cannot serve as an indication for leptoquarks' existence.

On the other hand, for the gateway red leptoquarks we may have something more interesting like this:

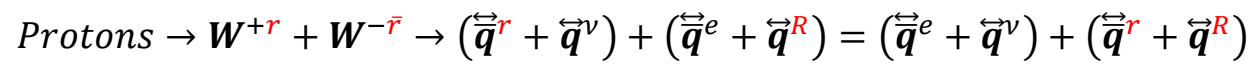

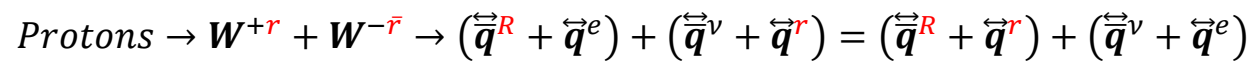

Here we do not have an outgoing stream of fermion/anti-fermion pairs, and one may suspect that this can serve as an indication of some new process. Unfortunately, (3.3-13) and (3.3-14) are, respectively, completely equivalent to the following two processes:

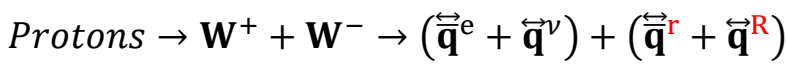

$$
\begin{aligned}
& \text { Protons } \rightarrow \mathbf{W}^{+}+\mathbf{W}^{-} \rightarrow\left(\overleftrightarrow{\mathbf{q}}^{\mathrm{R}}+\overleftrightarrow{\mathbf{q}}^{\mathrm{r}}\right)+\left(\left(\overleftrightarrow{\mathbf{q}}^{v}+\overleftrightarrow{\mathbf{q}}^{e}\right)\right.
\end{aligned}
$$

Furthermore, as we shall prove in section 4.6, all the charged $\mathbf{W}$ bosons have precisely the same mass. Hence, we conclude that (3.3-13) and (3.3-14) are indistinguishable from (3.3-15) and (3.3-16).

Thus, we have proved that trying to detect leptoquarks via pair production is a futile exercise, as indeed confirmed experimentally. 


\section{Unified Charge Vectors Theory}

\section{The Unified Lagrangian}

In this chapter, we integrate all the results obtained so far and construct the unified Lagrangian.

We then compute various coupling constants, including the weak mixing angle $\theta$.

Finally, we compute the masses of all the gauge bosons. In particular, we prove that all the $\mathrm{W}$ bosons appearing in the bosons periodic table have precisely the same mass. 


\section{Unified Charge Vectors Theory}

\subsection{The Unified Connections Fields}

With the help of the bosons periodic table (3.3-7), we construct the following Unified Connections Fields:

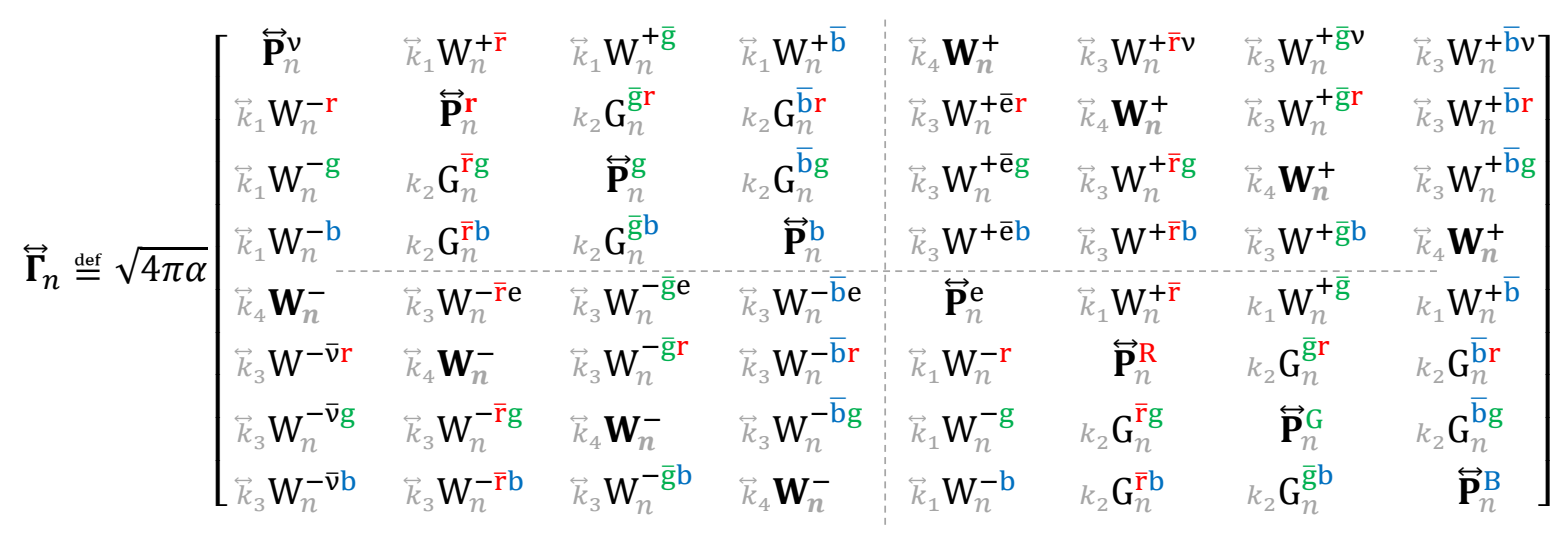

Where $\overleftarrow{\Gamma}_{n}$ and $\vec{\Gamma}_{n}$ are two $8 \times 8$ matrices, each containing 64 spacetime-vectors, representing the various bosonsfields. Since $\overleftarrow{\Gamma}_{n}$ and $\vec{\Gamma}_{n}$ have the structure of a geometric connection field, we refer to them as the unified connections fields. The symbols $\overleftrightarrow{k}_{1}, k_{2}, \overleftrightarrow{k}_{3}, \overleftrightarrow{k}_{4}$ stand for various coupling coefficients, which we shall compute later on. Along the diagonal, we have the potential bosons fields, denoted collectively by $\overleftrightarrow{\mathrm{P}}_{n}^{\mathrm{x}}$. To construct the exact definition of these fields, we refer back to (2.10-12) to (2.10-15) and set:

$$
\begin{aligned}
& {\left[\begin{array}{cccc}
\overleftarrow{\mathrm{P}}_{n}^{v} & 0 & 0 & 0 \\
0 & \overleftarrow{\mathrm{P}}_{n}^{\mathrm{r}} & 0 & 0 \\
0 & 0 & \overleftarrow{\mathrm{P}}_{n}^{\mathrm{g}} & 0 \\
0 & 0 & 0 & \overleftarrow{\mathrm{P}}_{n}^{\mathrm{b}}
\end{array}\right] \stackrel{\text { def }}{=} \frac{\mathrm{B}_{n}}{2 \theta_{\mathrm{c}}} \mathfrak{T}+\frac{\mathrm{W}_{n}}{2 \theta_{\mathrm{s}}} \mathbf{I}+\frac{2 \sqrt{2}}{3}\left[\begin{array}{cccc}
0 & 0 & 0 & 0 \\
0 & \mathrm{~S}_{n}^{\mathrm{r}} & 0 & 0 \\
0 & 0 & \mathrm{~S}_{n}^{\mathrm{g}} & 0 \\
0 & 0 & 0 & \mathrm{~S}_{n}^{\mathrm{b}}
\end{array}\right]} \\
& {\left[\begin{array}{cccc}
\mathrm{P}_{n}^{\mathrm{e}} & 0 & 0 & 0 \\
0 & \overleftarrow{\mathrm{P}}_{n}^{\mathrm{R}} & 0 & 0 \\
0 & 0 & \overleftarrow{\mathrm{P}}_{n}^{\mathrm{G}} & 0 \\
0 & 0 & 0 & \overleftarrow{\mathrm{P}}_{n}^{\mathrm{B}}
\end{array}\right] \stackrel{\text { def }}{=} \frac{\mathrm{B}_{n}}{2 \theta_{\mathrm{c}}} \mathfrak{T}-\frac{\mathrm{W}_{n}}{2 \theta_{\mathrm{s}}} \mathbf{I}+\frac{2 \sqrt{2}}{3}\left[\begin{array}{cccc}
0 & 0 & 0 & 0 \\
0 & \mathrm{~S}_{n}^{\mathrm{r}} & 0 & 0 \\
0 & 0 & \mathrm{~S}_{n}^{\mathrm{g}} & 0 \\
0 & 0 & 0 & \mathrm{~S}_{n}^{\mathrm{b}}
\end{array}\right]}
\end{aligned}
$$

And

$$
\begin{aligned}
& {\left[\begin{array}{cccc}
\overrightarrow{\mathrm{P}}_{n}^{v} & 0 & 0 & 0 \\
0 & \overrightarrow{\mathrm{P}}_{n}^{\mathrm{r}} & 0 & 0 \\
0 & 0 & \overrightarrow{\mathrm{P}}_{n}^{\mathrm{g}} & 0 \\
0 & 0 & 0 & \overrightarrow{\mathrm{P}}_{n}^{\mathrm{b}}
\end{array}\right] \stackrel{\text { def }}{=} \frac{\mathrm{B}_{n}}{2 \theta_{\mathrm{c}}} \mathfrak{T}+\frac{\mathrm{B}_{n}}{2 \theta_{\mathrm{c}}} \mathbf{I}+\frac{2 \sqrt{2}}{3}\left[\begin{array}{cccc}
0 & 0 & 0 & 0 \\
0 & \mathrm{~S}_{n}^{\mathrm{r}} & 0 & 0 \\
0 & 0 & \mathrm{~S}_{n}^{\mathrm{g}} & 0 \\
0 & 0 & 0 & \mathrm{~S}_{n}^{\mathrm{b}}
\end{array}\right]} \\
& {\left[\begin{array}{cccc}
\overrightarrow{\mathrm{P}}_{n}^{\mathrm{e}} & 0 & 0 & 0 \\
0 & \overrightarrow{\mathrm{P}}_{n}^{\mathrm{R}} & 0 & 0 \\
0 & 0 & \overrightarrow{\mathrm{P}}_{n}^{\mathrm{G}} & 0 \\
0 & 0 & 0 & \overrightarrow{\mathrm{P}}_{n}^{\mathrm{B}}
\end{array}\right] \stackrel{\text { def }}{=} \frac{\mathrm{B}_{n}}{2 \theta_{\mathrm{c}}} \mathfrak{T}-\frac{\mathrm{B}_{n}}{2 \theta_{\mathrm{c}}} \mathbf{I}+\frac{2 \sqrt{2}}{3}\left[\begin{array}{cccc}
0 & 0 & 0 & 0 \\
0 & \mathrm{~S}_{n}^{\mathrm{r}} & 0 & 0 \\
0 & 0 & \mathrm{~S}_{n}^{\mathrm{g}} & 0 \\
0 & 0 & 0 & \mathrm{~S}_{n}^{\mathrm{b}}
\end{array}\right]}
\end{aligned}
$$




\section{Unified Charge Vectors Theory}

Great care must be taken not to confuse the spacetime vector fields $\mathrm{B}_{n}, \mathrm{~W}_{n}, \mathrm{~S}_{n}^{\mathrm{r}}, \mathrm{S}_{n}^{\mathrm{g}}, \mathrm{S}_{n}^{\mathrm{b}}$ with the corresponding constant charge directions $\widehat{\mathbf{B}}, \widehat{\mathbf{W}}, \widehat{\mathbf{S}}^{\mathrm{r}}, \widehat{\mathbf{S}}^{\mathrm{g}}, \widehat{\mathbf{S}}^{\mathrm{b}}$, for these are two sets of entirely different entities.

Next, we use (2.4-13) and define the $\mathbf{G}_{n}$ matrix as follows:

$$
\mathbf{G}_{n} \stackrel{\text { def }}{=}\left[\begin{array}{ccccc}
0 & 0 & 0 & 0 \\
0 & \frac{2 \sqrt{2}}{3} \mathbf{S}_{n}^{\mathrm{r}} & k_{2} \mathbf{G}_{n}^{\overline{\mathrm{g} r}} & k_{2} \mathbf{G}_{n}^{\overline{\mathrm{br}}} \\
0 & k_{2} \mathbf{G}_{n}^{\overline{\mathrm{rg}}} & \frac{2 \sqrt{2}}{3} \mathbf{S}_{n}^{\mathrm{g}} & k_{2} \mathbf{G}_{n}^{\overline{\mathrm{bg}}} \\
0 & k_{2} \mathbf{G}_{n}^{\overline{\mathrm{rb}}} & k_{2} \mathbf{G}_{n}^{\overline{\mathrm{gb}}} & \frac{2 \sqrt{2}}{3} \mathbf{S}_{n}^{\mathrm{b}}
\end{array}\right]=\left[\begin{array}{cccc}
0 & 0 & 0 & 0 \\
0 & \frac{2 \sqrt{2}}{3} \mathbf{G}_{n}^{\perp} & k_{2} \mathbf{G}_{n}^{\overline{\mathrm{gr}}} & k_{2} \mathbf{G}_{n}^{\overline{\mathrm{b}} \mathrm{r}} \\
0 & k_{2} \mathbf{G}_{n}^{\overline{\mathrm{r} g}} & -\frac{\sqrt{2}}{3}\left(\sqrt{3} \mathbf{G}_{n}^{+}+\mathbf{G}_{n}^{\perp}\right) & k_{2} \mathbf{G}_{n}^{\overline{\mathrm{b} g}} \\
0 & k_{2} \mathbf{G}_{n}^{\overline{\mathrm{r}} \mathrm{b}} & k_{2} \mathbf{G}_{n}^{\overline{\mathrm{gb}}} & \frac{\sqrt{2}}{3}\left(\sqrt{3} \mathbf{G}_{n}^{\vdash}-\mathbf{G}_{n}^{\perp}\right)
\end{array}\right]
$$

We also define the matrices $\overleftrightarrow{\mathbf{W}}_{n}$ and $\overleftrightarrow{\mathbf{W}}_{n}^{ \pm}$as follows:

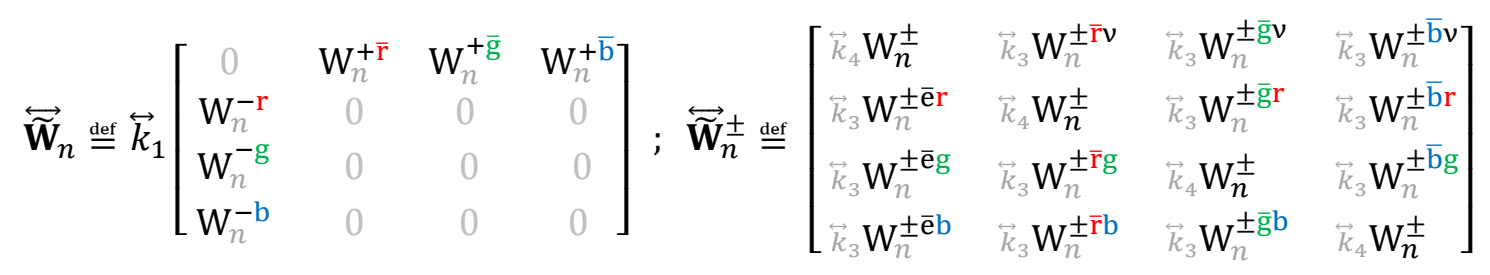

We can now significantly simplify (4.1-1) by rewriting it as follows:

And:

$$
\frac{\overleftarrow{\Gamma}_{n}}{\sqrt{4 \pi \alpha}} \stackrel{\text { def }}{=} \frac{\mathrm{B}_{n}}{2 \theta_{\mathrm{c}}}\left[\begin{array}{cc}
\mathfrak{T} & 0 \\
0 & \mathfrak{T}
\end{array}\right]+\left[\begin{array}{cc}
\mathbf{G}_{n} & 0 \\
0 & \mathbf{G}_{n}
\end{array}\right]+\frac{\mathbf{W}_{n}}{2 \theta_{\mathbf{s}}}\left[\begin{array}{cc}
\mathbf{I} & 0 \\
0 & -\mathbf{I}
\end{array}\right]+\left[\begin{array}{ll}
\overleftarrow{\mathbf{W}}_{n} & \overleftarrow{\mathbf{W}}_{n}^{+} \\
\widetilde{\mathbf{W}}_{n}^{-} & \overleftarrow{\mathbf{W}}_{n}
\end{array}\right]
$$

$$
\frac{\vec{\Gamma}_{n}}{\sqrt{4 \pi \alpha}} \stackrel{\text { def }}{=} \frac{\mathrm{B}_{n}}{2 \theta_{\mathrm{c}}}\left[\begin{array}{cc}
\mathfrak{T} & 0 \\
0 & \mathfrak{T}
\end{array}\right]+\left[\begin{array}{cc}
\mathbf{G}_{n} & 0 \\
0 & \mathbf{G}_{n}
\end{array}\right]+\frac{\mathrm{B}_{n}}{2 \theta_{\mathrm{c}}}\left[\begin{array}{rr}
\mathbf{I} & 0 \\
0 & -\mathbf{I}
\end{array}\right]+\left[\begin{array}{ll}
\overrightarrow{\widetilde{\mathbf{W}}}_{n} & \overrightarrow{\widetilde{\mathbf{W}}}_{n}^{+} \\
\overrightarrow{\widetilde{\mathbf{W}}}_{n}^{-} & \overrightarrow{\widetilde{\mathbf{W}}}_{n}
\end{array}\right]
$$

Where:

$$
\boldsymbol{T} \stackrel{\text { def }}{=}\left[\begin{array}{cccc}
-1 & 0 & 0 & 0 \\
0 & \frac{1}{3} & 0 & 0 \\
0 & 0 & \frac{1}{3} & 0 \\
0 & 0 & 0 & \frac{1}{3}
\end{array}\right] \quad \text { and } \quad \mathbf{I} \stackrel{\text { def }}{=}\left[\begin{array}{llll}
1 & 0 & 0 & 0 \\
0 & 1 & 0 & 0 \\
0 & 0 & 1 & 0 \\
0 & 0 & 0 & 1
\end{array}\right]
$$




\section{Unified Charge Vectors Theory}

\subsection{The Unified Spinors Fields}

Next, we define the following left and right Unified Spinors Fields, representing respectively the neutrinos, the dark quarks, the electrons, and the bright quarks:

$$
\overleftrightarrow{\boldsymbol{\Psi}} \stackrel{\text { def }}{=}\left[\overleftrightarrow{\psi}^{v}, \overleftrightarrow{\psi}^{\mathrm{r}}, \overleftrightarrow{\psi}^{\mathrm{g}}, \overleftrightarrow{\psi}^{\mathrm{b}}, \overleftrightarrow{\psi}^{e}, \overleftrightarrow{\psi}^{\mathrm{R}}, \overleftrightarrow{\psi}^{\mathrm{G}}, \overleftrightarrow{\psi}^{\mathrm{B}}\right]^{\mathrm{T}}
$$

Where each of the $\overleftrightarrow{\psi}^{\mathrm{x}}$ is a spinor field.

We can now formulate the following Unified Dirac Equations.

Where:

$$
i \overleftarrow{\Sigma}^{\delta}\left(\overleftarrow{\square}_{\delta} \overleftarrow{\Psi}\right)=\mathbf{M} \vec{\Psi} ; i \vec{\Sigma}^{\delta}\left(\vec{\square}_{\delta} \vec{\Psi}\right)=\mathbf{M}^{\dagger} \overleftarrow{\Psi}
$$

$$
\begin{aligned}
& \overleftrightarrow{\square}_{n} \overleftrightarrow{\boldsymbol{\Psi}} \stackrel{\text { def }}{=}\left(\partial_{n}+i \overleftrightarrow{\boldsymbol{\Gamma}}_{n}\right) \overleftrightarrow{\mathbf{\Psi}} \\
& \overleftrightarrow{\boldsymbol{\Sigma}}^{n} \stackrel{\text { def }}{=}\left[\begin{array}{llllllll}
\overleftrightarrow{\boldsymbol{\sigma}}^{n} & 0 & 0 & 0 & 0 & 0 & 0 & 0 \\
0 & \overleftrightarrow{\boldsymbol{\sigma}}^{n} & 0 & 0 & 0 & 0 & 0 & 0 \\
0 & 0 & \overleftrightarrow{\boldsymbol{\sigma}}^{n} & 0 & 0 & 0 & 0 & 0 \\
0 & 0 & 0 & \overleftrightarrow{\boldsymbol{\sigma}}^{n} & 0 & 0 & 0 & 0 \\
0 & 0 & 0 & 0 & \overleftrightarrow{\boldsymbol{\sigma}}^{n} & 0 & 0 & 0 \\
0 & 0 & 0 & 0 & 0 & \overleftrightarrow{\boldsymbol{\sigma}}^{n} & 0 & 0 \\
0 & 0 & 0 & 0 & 0 & 0 & \overleftrightarrow{\boldsymbol{\sigma}}^{n} & 0 \\
0 & 0 & 0 & 0 & 0 & 0 & 0 & \overleftrightarrow{\boldsymbol{\sigma}}^{n}
\end{array}\right] \\
& \overleftrightarrow{\boldsymbol{\sigma}}^{0} \stackrel{\text { def }}{=}\left[\begin{array}{ll}
1 & 0 \\
0 & 1
\end{array}\right] ; \quad \overleftrightarrow{\boldsymbol{\sigma}}^{1} \stackrel{\text { deff }}{=}\left[\begin{array}{cc}
0 & \stackrel{\leftrightarrow}{1} \\
\stackrel{1}{1} & 0
\end{array}\right] ; \quad \stackrel{\boldsymbol{\sigma}}{ }^{2} \stackrel{\text { def }}{=} i\left[\begin{array}{cc}
0 & -\overleftrightarrow{1} \\
\overleftrightarrow{1} & 0
\end{array}\right] ; \quad \overleftrightarrow{\boldsymbol{\sigma}}^{3} \stackrel{\text { def }}{=}\left[\begin{array}{cc}
\stackrel{\leftrightarrow}{1} & 0 \\
0 & -\overleftrightarrow{1}
\end{array}\right] \\
& \text { Where } \stackrel{\leftarrow}{\stackrel{\text { def }}{=}}-1 ; \overrightarrow{1} \stackrel{\text { def }}{=}+1
\end{aligned}
$$

And $\mathbf{M}$ is some $8 \times 8$ matrix called the Mass Field, which we shall explore in the next section. 


\section{Unified Charge Vectors Theory}

Next, we want to investigate the way the unified Dirac equations transform under the following two general transformations:

$$
\overleftrightarrow{\Psi}=\overleftarrow{\Lambda} \overleftarrow{\Psi} \quad \text { and } \quad \dot{\vec{\Psi}}=\vec{\Lambda} \vec{\Psi} \text { or collectively } \dot{\boldsymbol{\Psi}}=\overleftrightarrow{\Lambda} \overleftrightarrow{\Psi}
$$

Where $\overleftarrow{\Lambda}(x)$ and $\vec{\Lambda}(x)$ are some two (possibly different) $8 \times 8$ invertible matrix fields. We shall denote the inverse transformations by $\overleftrightarrow{\Lambda}$, i.e.:

$$
\overleftrightarrow{\overleftarrow{\Lambda}} \stackrel{\text { def }}{=}(\overleftrightarrow{\Lambda})^{-1} \text { and hence } \stackrel{\leftrightarrow}{\Psi}=\overleftrightarrow{\Lambda} \overleftrightarrow{\mathbf{\Psi}}
$$

Differentiating (4.2-7), we obtain:

And hence:

$$
\partial_{n} \overleftrightarrow{\boldsymbol{\Psi}}=\overleftrightarrow{\bar{\Lambda}}\left(\partial_{n} \overleftrightarrow{\mathbf{\Psi}}\right)+\left(\partial_{n} \overleftrightarrow{\mathbf{\Lambda}}\right) \overleftrightarrow{\mathbf{\Psi}}=\overleftrightarrow{\bar{\Lambda}}\left(\partial_{n}+\overleftrightarrow{\Lambda} \partial_{n} \overleftrightarrow{\mathbf{\Lambda}}\right) \overleftrightarrow{\mathbf{\Psi}}
$$

$$
\overleftrightarrow{\square}_{n} \overleftrightarrow{\boldsymbol{\Psi}} \stackrel{\text { def }}{=}\left(\partial_{n}+i \overleftrightarrow{\boldsymbol{\Gamma}_{n}}\right) \overleftrightarrow{\boldsymbol{\Psi}}=\overleftrightarrow{\boldsymbol{\Lambda}}\left(\partial_{n}+i\left(\overleftrightarrow{\Lambda} \overleftrightarrow{\boldsymbol{\Gamma}}_{n} \overleftrightarrow{\boldsymbol{\Lambda}}-i \overleftrightarrow{\Lambda} \partial_{n} \overleftrightarrow{\boldsymbol{\Lambda}}\right)\right) \overleftrightarrow{\mathbf{\Psi}}
$$

This motivates us to define the transformed connection $\stackrel{\vec{\Gamma}}{n}_{n}$, as follows:

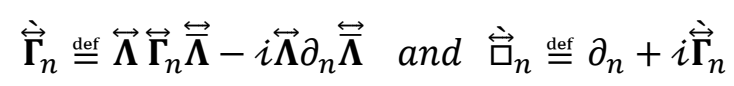

So:

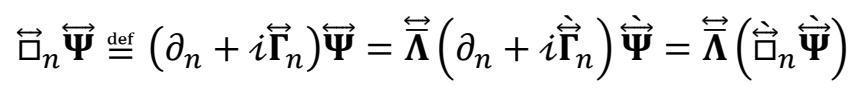

Plugging this in the unified Dirac equations (4.2-2), we obtain:

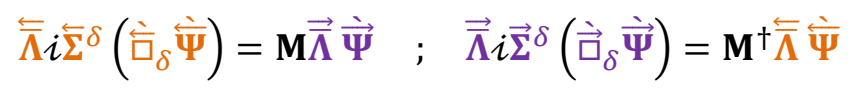

Multiplying these equations, respectively, from the left by $\overleftarrow{\Lambda}$ and $\vec{\Lambda}$, we conclude that:

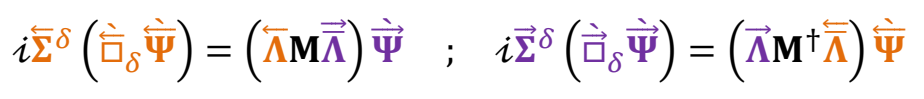

Or, using the relation $\left(\vec{\Lambda} \mathbf{M}^{\dagger} \overleftarrow{\bar{\Lambda}}\right)=\left(\overleftarrow{\bar{\Lambda}}^{\dagger} \mathbf{M} \vec{\Lambda}^{\dagger}\right)^{\dagger}$ :

$$
i \overleftarrow{\Sigma}^{\delta}\left(\overleftarrow{\square}_{\delta} \dot{\Psi}\right)=(\overleftarrow{\Lambda} \mathbf{M} \overrightarrow{\bar{\Lambda}}) \dot{\vec{\Psi}} \quad ; \quad i \vec{\Sigma}^{\delta}\left(\dot{\vec{\square}}_{\delta} \dot{\vec{\Psi}}\right)=\left(\overleftarrow{\bar{\Lambda}}^{\dagger} \mathbf{M} \vec{\Lambda}^{\dagger}\right)^{\dagger} \overleftarrow{\Psi}
$$




\section{Unified Charge Vectors Theory}

Comparing (4.2-14) to (4.2-2), we conclude that in order for the unified Dirac equations to be invariant under the transformations $\overleftrightarrow{\Lambda}$, we must have:

$$
\overleftarrow{\Lambda} \mathbf{M} \vec{\Lambda}=\overleftarrow{\Lambda}^{\dagger} \mathbf{M} \vec{\Lambda}^{\dagger}
$$

This suggests we restrict ourselves to unitary transformations. i.e., transformations satisfying:

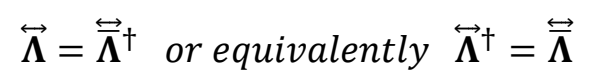

For such transformations, we can define:

$$
\grave{\mathbf{M}} \stackrel{\text { def }}{=} \overleftarrow{\Lambda} \mathbf{M} \vec{\Lambda} \quad \text { so } \quad \grave{\mathbf{M}}^{\dagger}=\vec{\Lambda} \mathbf{M}^{\dagger} \overleftarrow{\bar{\Lambda}}
$$

Plugging this in (4.2-13), we finally obtain:

$$
i \overleftarrow{\Sigma}^{\delta}\left(\grave{\square}_{\delta} \dot{\Psi}\right)=\grave{\mathbf{M}} \dot{\vec{\Psi}} \quad ; \quad i \vec{\Sigma}^{\delta}\left(\dot{\vec{\square}}_{\delta} \dot{\vec{\Psi}}\right)=\grave{\mathbf{M}}^{\dagger}
$$

We thus conclude that the unified Dirac equations are invariant under $8 \times 8$ unitary transformations $\overleftrightarrow{\boldsymbol{\Lambda}}$, with respect to the following transformation laws:

$$
\begin{aligned}
& \overleftrightarrow{\Psi}=\overleftrightarrow{\Lambda} \overleftrightarrow{\Psi}
\end{aligned}
$$

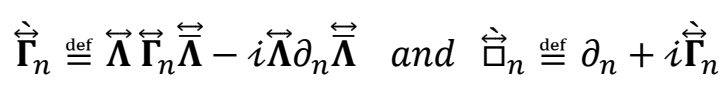

$$
\begin{aligned}
& \grave{\mathbf{M}} \stackrel{\text { def }}{=} \overleftarrow{\Lambda} \mathbf{M} \overrightarrow{\bar{\Lambda}}
\end{aligned}
$$

The set of all such transformations is usually referred to as $\mathrm{U}(8)$. 


\section{Unified Charge Vectors Theory}

With the help of the unified spinors field $\overleftrightarrow{\boldsymbol{\Psi}}$, we can now define the Multi-Gen Spinors Field $\left[\begin{array}{l}\overleftrightarrow{\Psi}_{1} \\ \overleftrightarrow{\Psi}_{2} \\ \overleftrightarrow{\Psi}_{3}\end{array}\right]$, where "Multi-Gen" stands for multi-generations, and $\overleftrightarrow{\Psi}_{1}, \overleftrightarrow{\mathbf{\Psi}}_{2}, \overleftrightarrow{\Psi}_{3}$ are, respectively, the unified spinors fields associated with each of the three known generations of fermions.

The corresponding Multi-Gen Dirac Equations are given by:

$$
i \overleftarrow{\Sigma}^{\delta} \overleftarrow{\square}_{\delta}\left[\begin{array}{l}
\overleftarrow{\Psi}_{1} \\
\overleftarrow{\Psi}_{2} \\
\overleftarrow{\Psi}_{3}
\end{array}\right]=\left[\begin{array}{lll}
\mathbf{M}_{11} & 0 & 0 \\
0 & \mathbf{M}_{22} & 0 \\
0 & 0 & \mathbf{M}_{33}
\end{array}\right]\left[\begin{array}{l}
\vec{\Psi}_{1} \\
\vec{\Psi}_{2} \\
\vec{\Psi}_{3}
\end{array}\right] ; \quad i \vec{\Sigma}^{\delta} \vec{\square}_{\delta}\left[\begin{array}{l}
\vec{\Psi}_{1} \\
\vec{\Psi}_{2} \\
\vec{\Psi}_{3}
\end{array}\right]=\left[\begin{array}{lll}
\mathbf{M}_{11}^{\dagger} & 0 & 0 \\
0 & \mathbf{M}_{22}^{\dagger} & 0 \\
0 & 0 & \mathbf{M}_{33}^{\dagger}
\end{array}\right]\left[\begin{array}{l}
\overleftarrow{\Psi}_{1} \\
\overleftarrow{\Psi}_{2} \\
\overleftarrow{\Psi}_{3}
\end{array}\right]
$$

Where:

$$
\overleftrightarrow{\mathrm{D}}_{n}\left[\begin{array}{l}
\overleftrightarrow{\mathbf{\Psi}}_{1} \\
\overleftrightarrow{\boldsymbol{\Psi}}_{2} \\
\overleftrightarrow{\mathbf{\Psi}}_{3}
\end{array}\right] \stackrel{\text { def }}{=}\left(\partial_{n}+i\left[\begin{array}{ccc}
\overleftrightarrow{\boldsymbol{\Gamma}}_{n} & 0 & 0 \\
0 & \overleftrightarrow{\boldsymbol{\Gamma}}_{n} & 0 \\
0 & 0 & \overleftrightarrow{\boldsymbol{\Gamma}}_{n}
\end{array}\right]\right)\left[\begin{array}{l}
\overleftrightarrow{\mathbf{\Psi}}_{1} \\
\overleftrightarrow{\mathbf{\Psi}}_{2} \\
\overleftrightarrow{\mathbf{\Psi}}_{3}
\end{array}\right]
$$

Since experimental evidence suggests that the fermions we measure are actually linear combinations of the three generations, we define the Mixed Spinors Field as follows:

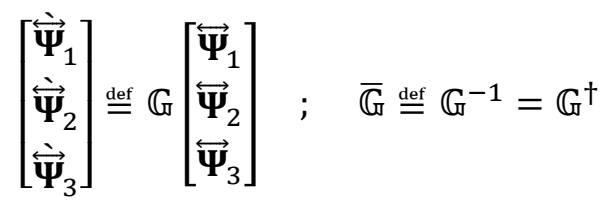

Where $\mathbb{G}$ is some constant $24 \times 24$ unitary matrix, which represents the mixing of the various generations. With the help of $\mathbb{G}$, we defined the Mixed Mass Field and Mixed Connections Fields as follows:

$$
\mathbb{M} \stackrel{\text { def }}{=} \mathbb{G}\left[\begin{array}{lll}
\mathbf{M}_{11} & 0 & 0 \\
0 & \mathbf{M}_{22} & 0 \\
0 & 0 & \mathbf{M}_{33}
\end{array}\right] \overline{\mathbb{G}} \quad \text { and } \quad \overleftrightarrow{\mathbf{Y}}_{n} \stackrel{\text { def }}{=} \mathbb{G}\left[\begin{array}{lll}
\overleftrightarrow{\boldsymbol{\Gamma}}_{n} & 0 & 0 \\
0 & \overleftrightarrow{\boldsymbol{\Gamma}}_{n} & 0 \\
0 & 0 & \overleftrightarrow{\boldsymbol{\Gamma}}_{n}
\end{array}\right] \overline{\mathbb{G}}
$$

This allows us to write the Mixed Dirac Equations as:

Where:

$$
i \overleftarrow{\Sigma}^{\delta} \dot{\square}_{\delta}\left[\begin{array}{c}
\dot{\Psi}_{1} \\
\dot{\Psi}_{2} \\
\dot{\Psi}_{3}
\end{array}\right]=\mathbb{M}\left[\begin{array}{c}
\dot{\vec{\Psi}}_{1} \\
\dot{\Psi}_{2} \\
\dot{\Psi}_{3}
\end{array}\right] ; \quad i \vec{\Sigma} \delta \dot{\vec{\square}}_{\delta}\left[\begin{array}{c}
\dot{\vec{\Psi}}_{1} \\
\dot{\vec{\Psi}}_{2} \\
\dot{\vec{\Psi}}_{3}
\end{array}\right]=\mathbb{M}^{\dagger}\left[\begin{array}{c}
\dot{\Psi}_{1} \\
\dot{\Psi}_{2} \\
\dot{\Psi}_{3}
\end{array}\right]
$$

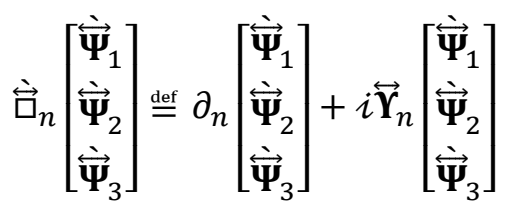




\section{Unified Charge Vectors Theory}

\subsection{The Unified Mass Field}

The transformation law of $\mathbf{M}$ described in (4.2-21) suggests that the mass matrix is actually a matrix field. That is to say, since the transformations matrices $\overleftrightarrow{\Lambda}$ are fields, then so must $\mathbf{M}$ be.

We also would like $\mathbf{M}$ to be diagonalizable. That is to say, we assume there exist some Mass Diagonalizing Unitary Transformations $\overleftrightarrow{\Lambda}$, such that:

$$
\mathbf{M} \backslash \stackrel{\text { def }}{=} \overleftarrow{\Lambda} \mathbf{M} \overrightarrow{\bar{\Lambda}}=\left[\begin{array}{llll}
\mathrm{M}_{1}^{1 \backslash} & 0 & \ldots & 0 \\
0 & \mathrm{M}_{2}^{2 \backslash} & \ldots & 0 \\
\vdots & \vdots & \ddots & 0 \\
0 & 0 & 0 & \mathrm{M}_{8}^{8 \backslash}
\end{array}\right]
$$

Where the backslash sign in $\mathbf{M} \backslash$ denotes a diagonal matrix, and $\mathrm{M}_{n}^{n \backslash}$ is the $\mathrm{n}^{\text {th }}$ element in the diagonal.

This diagonalization requirement comes from the need to uncouple the unified Dirac equations (4.2-2) into eight different equations for eight different particles, each with its own distinctive mass term given by the corresponding diagonal element $M_{1}^{1 \backslash}, \ldots, M_{8}^{8 \backslash}$.

Also, since the fields $M_{1}^{1 \backslash}, \ldots, M_{8}^{8 \backslash}$ have to function as mass terms, they must be strictly positive. This means that there exist some eight fields $\lambda_{1}^{1}, \ldots, \lambda_{8}^{8}$, such that:

$$
\mathrm{M}_{n}^{n \backslash}=e^{\lambda_{n}^{n}} \quad \text { where } \quad \lambda_{n}^{n}=\ln \left(\mathrm{M}_{n}^{n \backslash}\right)
$$

Or in matrix form:

$$
\mathbf{M} \backslash=e^{\lambda} \text { where } \lambda \stackrel{\text { def }}{=}\left[\begin{array}{llll}
\lambda_{1}^{1} & 0 & \ldots & 0 \\
0 & \lambda_{2}^{2} & \ldots & 0 \\
\vdots & \vdots & \ddots & 0 \\
0 & 0 & 0 & \lambda_{8}^{8}
\end{array}\right]
$$

Hence, the most general form of $\mathbf{M}$ is given by:

$$
\mathbf{M}=\overleftarrow{\Lambda} \mathbf{M} \backslash \overrightarrow{\bar{\Lambda}}=\overleftarrow{\Lambda} e^{\lambda \overrightarrow{\bar{\Lambda}}}
$$




\section{Unified Charge Vectors Theory}

Next, we make the simplifying assumption that the fields $\lambda_{1}^{1}, \ldots, \lambda_{8}^{8}$ can all be expressed as simple linear functions of a more fundamental scalar field $\phi$, as follows:

$$
\lambda_{n}^{n}(\phi)=\phi+\ell_{n}^{n} \quad \text { so } \quad \lambda(\phi)=\mathbf{I} \phi+\boldsymbol{\ell}
$$

Where $\ell_{1}^{1}, \ldots, \ell_{8}^{8}$ are some eight coefficients.

With this, we can rewrite (4.3-4) as follows:

$$
\mathbf{M}(\phi)=\overleftarrow{\Lambda} e^{\lambda(\phi)} \overrightarrow{\bar{\Lambda}}
$$

In particular, we have:

$$
\mathbf{M}(0)=\overleftarrow{\Lambda} e^{\lambda(0)} \overrightarrow{\bar{\Lambda}}=\overleftarrow{\Lambda} \boldsymbol{m} \backslash \vec{\Lambda}
$$

Where we define:

$$
\boldsymbol{m} \backslash \stackrel{\text { def }}{=} e^{\lambda(0)}=e^{\ell}=\left[\begin{array}{llll}
e^{\ell_{1}^{1}} & 0 & \ldots & 0 \\
0 & e^{\ell_{2}^{2}} & \ldots & 0 \\
\vdots & \vdots & \ddots & 0 \\
0 & 0 & 0 & e^{\ell_{8}^{8}}
\end{array}\right]
$$

We shall refer to the constant matrix $\boldsymbol{m} \backslash$ as the Fermions Mass Matrix.

With the help of $\boldsymbol{m} \backslash$, we can rewrite (4.3-6) as follows:

$$
\mathbf{M}(\phi)=\overleftarrow{\Lambda} e^{\lambda(\phi)-\ell+\ell} \overrightarrow{\bar{\Lambda}}=\overleftarrow{\Lambda} e^{\mathbf{I} \phi+\ell} \overrightarrow{\bar{\Lambda}}=\overleftarrow{\Lambda} e^{\mathbf{I} \phi} e^{\ell} \overrightarrow{\bar{\Lambda}}=\overleftarrow{\Lambda} \boldsymbol{\Phi} \backslash \boldsymbol{m} \backslash \overrightarrow{\bar{\Lambda}}
$$

Where we define:

$$
\boldsymbol{\Phi} \backslash(\phi) \stackrel{\text { def }}{=} e^{\mathbb{I} \phi}=\mathbb{I} e^{\phi} \text { where } \mathbb{I} \text { is the } 8 \times 8 \text { identity matrix }
$$

This implies that:

$$
\boldsymbol{\Phi} \backslash(0)=\mathbb{I}
$$




\section{Unified Charge Vectors Theory}

Using the identity $\vec{\Lambda} \vec{\Lambda}=\mathbb{I}$, we notice that we can rewrite (4.3-9) as follows:

Where we define:

$$
\mathbf{M}(\phi)=\overleftarrow{\Lambda} \boldsymbol{\Phi} \backslash \boldsymbol{m} \backslash \overrightarrow{\bar{\Lambda}}=(\overleftarrow{\Lambda} \boldsymbol{\Phi} \backslash \overrightarrow{\bar{\Lambda}})(\vec{\Lambda} \boldsymbol{m} \backslash \vec{\Lambda})=\boldsymbol{\Phi} \vec{m}
$$

$$
\boldsymbol{\Phi} \stackrel{\text { def }}{=} \overleftarrow{\Lambda} \mathbf{\Phi} \backslash \vec{\Lambda} \text { and } \vec{m} \stackrel{\text { def }}{=} \vec{\Lambda} \backslash \overrightarrow{\bar{\Lambda}}
$$

On the other hand, since $\boldsymbol{\Phi} \backslash$ and $\boldsymbol{m} \backslash$ are diagonal, we have $\boldsymbol{\Phi} \backslash \boldsymbol{m} \backslash=\boldsymbol{m} \backslash \boldsymbol{\Phi} \backslash$, and so we can also write:

Where:

$$
\mathbf{M}(\phi)=\overleftarrow{\Lambda} \boldsymbol{m} \backslash \boldsymbol{\Phi} \backslash \overrightarrow{\bar{\Lambda}}=(\overleftarrow{\Lambda} \boldsymbol{m} \backslash \overleftarrow{\Lambda})(\overleftarrow{\Lambda} \boldsymbol{\Phi} \backslash \vec{\Lambda})=\overleftarrow{m} \boldsymbol{\Phi}
$$

$$
\overleftarrow{m} \stackrel{\text { def }}{=} \overleftarrow{\Lambda} \boldsymbol{m} \backslash \overleftarrow{\Lambda}
$$

This allows us to rewrite the unified Dirac equations (4.2-2) as follows:

$$
i \overleftarrow{\Sigma}^{\delta}\left(\overleftarrow{\square}_{\delta} \overleftarrow{\Psi}\right)=\overleftarrow{m} \boldsymbol{\Phi} \vec{\Psi} ; i \vec{\Sigma}^{\delta}\left(\vec{\square}_{\delta} \vec{\Psi}\right)=\overrightarrow{\boldsymbol{m}}^{\dagger} \boldsymbol{\Phi}^{\dagger} \overleftarrow{\Psi}
$$

We shall refer to $\boldsymbol{\Phi}$ as the Higgs Matrix Field.

Notice that for the identity transformations $\overleftrightarrow{\Lambda}=\mathbf{I},(4.3-16)$ becomes:

$$
i \overleftarrow{\Sigma}^{\delta}\left(\overleftarrow{\square}_{\delta} \overleftarrow{\Psi}\right)=\boldsymbol{m} \backslash \boldsymbol{\Phi} \backslash(\phi) \vec{\Psi} ; i \vec{\Sigma}^{\delta}\left(\vec{\square}_{\delta} \vec{\Psi}\right)=\boldsymbol{m} \backslash \boldsymbol{\Phi} \backslash *(\phi) \overleftarrow{\Psi}
$$




\section{Unified Charge Vectors Theory}

Next, we want to define the $\square_{n}$ operation for the Higgs matrix field, such that we have:

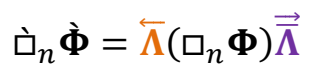

We, therefore, begin by setting $\grave{\mathbf{\Phi}} \stackrel{\text { def }}{=} \overleftarrow{\Lambda} \mathbf{\Phi} \vec{\Lambda}$, and compute $\partial_{n} \grave{\boldsymbol{\Phi}}$ as follows:

$$
\begin{gathered}
\partial_{n} \grave{\boldsymbol{\Phi}}=\partial_{n}(\overleftarrow{\Lambda} \boldsymbol{\Phi} \overrightarrow{\bar{\Lambda}})=\overleftarrow{\Lambda}\left(\partial_{n} \boldsymbol{\Phi}\right) \overrightarrow{\vec{\Lambda}}+\left(\partial_{n} \overleftarrow{\Lambda}\right) \boldsymbol{\Phi} \overrightarrow{\bar{\Lambda}}+\overleftarrow{\Lambda} \boldsymbol{\Phi}\left(\partial_{n} \overrightarrow{\bar{\Lambda}}\right) \\
=\overleftarrow{\Lambda}\left(\partial_{n} \boldsymbol{\Phi}+\left(\overleftarrow{\bar{\Lambda}} \partial_{n} \overleftarrow{\Lambda}\right) \boldsymbol{\Phi}+\boldsymbol{\Phi}\left(\partial_{n} \overrightarrow{\bar{\Lambda}}\right) \vec{\Lambda}\right) \overrightarrow{\bar{\Lambda}}
\end{gathered}
$$

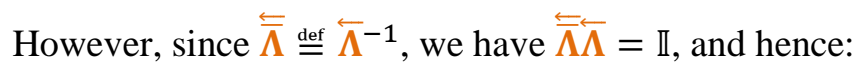

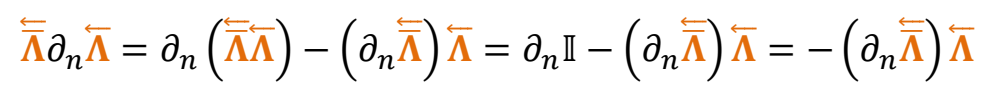

Plugging this back in (4.3-19), we conclude that:

$$
\partial_{n} \grave{\Phi}=\overleftarrow{\Lambda}\left(\partial_{n} \boldsymbol{\Phi}-\left(\partial_{n} \overleftarrow{\Lambda}\right) \overleftarrow{\Lambda} \boldsymbol{\Phi}+\boldsymbol{\Phi}\left(\partial_{n} \overrightarrow{\bar{\Lambda}}\right) \vec{\Lambda}\right) \overrightarrow{\bar{\Lambda}}
$$

This motivates us to define $\square_{n} \boldsymbol{\Phi}$ as follows:

$$
\square_{n} \boldsymbol{\Phi} \stackrel{\text { def }}{=} \partial_{n} \boldsymbol{\Phi}+i\left(\overleftarrow{\Gamma}_{n} \boldsymbol{\Phi}-\boldsymbol{\Phi} \vec{\Gamma}_{n}\right)
$$

To verify that (4.3-18) indeed holds, we use (4.3-21) to compute $\grave{d}_{n} \grave{\mathbf{\Phi}}$ as follows:

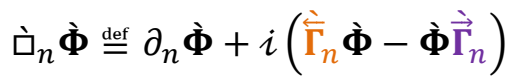

$$
\begin{aligned}
& =\overleftarrow{\Lambda}\left(\partial_{n} \boldsymbol{\Phi}-\left(\partial_{n} \overleftarrow{\bar{\Lambda}}\right) \overleftarrow{\Lambda} \boldsymbol{\Phi}+\boldsymbol{\Phi}\left(\partial_{n} \overrightarrow{\bar{\Lambda}}\right) \vec{\Lambda}\right) \overrightarrow{\bar{\Lambda}}+i\left(\overleftarrow{\Gamma}_{n} \grave{\boldsymbol{\Phi}}-\grave{\boldsymbol{\Phi}} \dot{\vec{\Gamma}}_{n}\right) \\
& =\overleftarrow{\Lambda}\left(\partial_{n} \boldsymbol{\Phi}-\left(\partial_{n} \overleftarrow{\Lambda}\right) \overleftarrow{\Lambda} \boldsymbol{\Phi}+\boldsymbol{\Phi}\left(\partial_{n} \overrightarrow{\bar{\Lambda}}\right) \vec{\Lambda}+i\left(\overleftarrow{\overleftarrow{\Lambda}} \bar{\Gamma}_{n} \grave{\boldsymbol{\Phi}} \vec{\Lambda}-\overleftarrow{\Lambda} \dot{\boldsymbol{\Phi}} \vec{\Gamma}_{n} \vec{\Lambda}\right)\right) \overrightarrow{\bar{\Lambda}}
\end{aligned}
$$

Substituting $\grave{\Phi} \stackrel{\text { def }}{=} \overleftarrow{\Lambda} \mathbf{\Phi} \overrightarrow{\bar{\Lambda}}$ and rearranging terms, we conclude that:

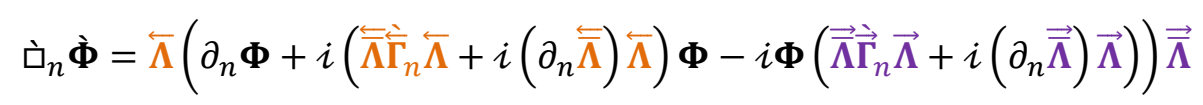

However, by (4.2-20) we have $\stackrel{\overleftarrow{\Lambda}}{\Gamma_{n}} \overleftarrow{\Lambda}+i\left(\partial_{n} \overleftarrow{\Lambda}\right) \overleftarrow{\Lambda}=\overleftarrow{\Gamma}_{n}$, and $\overrightarrow{\bar{\Lambda}}_{n} \vec{\Lambda}+i\left(\partial_{n} \overrightarrow{\bar{\Lambda}}\right) \vec{\Lambda}=\vec{\Gamma}_{n}$ so indeed we have:

$$
\grave{\dot{d}}_{n} \grave{\boldsymbol{\Phi}}=\overleftarrow{\Lambda}\left(\partial_{n} \boldsymbol{\Phi}+i\left(\overleftarrow{\Gamma}_{n} \boldsymbol{\Phi}-\boldsymbol{\Phi} \overrightarrow{\boldsymbol{\Gamma}}_{n}\right)\right) \overrightarrow{\bar{\Lambda}}=\overleftarrow{\Lambda}\left(\square_{n} \boldsymbol{\Phi}\right) \overrightarrow{\bar{\Lambda}}
$$




\section{Unified Charge Vectors Theory}

\subsection{The Unified Lagrangian}

We are now in a position to construct the following Single Generation Unified Lagrangian.

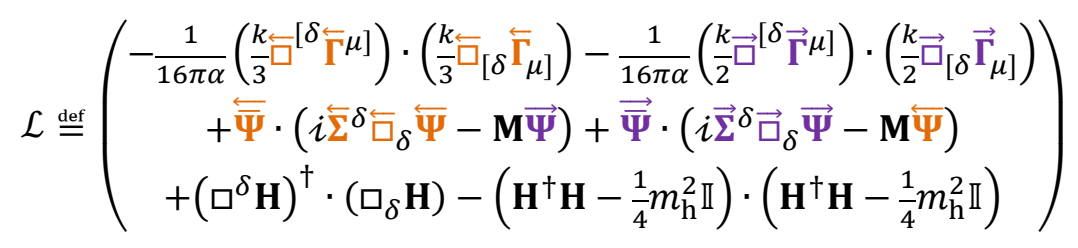

Where:

$$
\begin{aligned}
& \mathrm{X} \cdot \mathrm{Y} \stackrel{\text { def }}{=} \operatorname{Trace}(X Y) \\
& \overleftrightarrow{\square}_{[n} \overleftrightarrow{\boldsymbol{\Gamma}}_{m]} \stackrel{\text { def }}{=} \partial_{n} \overleftrightarrow{\boldsymbol{\Gamma}}_{m}-\partial_{m} \overleftrightarrow{\boldsymbol{\Gamma}}_{n}+\boldsymbol{i}\left(\overleftrightarrow{\boldsymbol{\Gamma}}_{n} \overleftrightarrow{\boldsymbol{\Gamma}}_{m}-\overleftrightarrow{\boldsymbol{\Gamma}}_{m} \overleftrightarrow{\boldsymbol{\Gamma}}_{n}\right)
\end{aligned}
$$

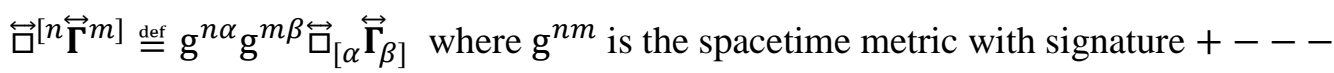

$$
\begin{aligned}
& {\overleftrightarrow{\square_{n}}}_{n} \overleftrightarrow{\boldsymbol{\Psi}} \stackrel{\text { def }}{=}\left(\partial_{n}+i \overleftrightarrow{\boldsymbol{\Gamma}}_{n}\right) \overleftrightarrow{\boldsymbol{\Psi}} \\
& \overleftrightarrow{\mathbf{\Psi}} \stackrel{\text { def }}{=}\left[\bar{\psi}_{1}, \ldots, \bar{\psi}_{8}\right] \text { where } \bar{\psi}_{n} \stackrel{\text { def }}{=} \psi_{n}^{\dagger} \gamma^{0} \\
& \mathbf{M}=\overleftarrow{\Lambda} \boldsymbol{m} \backslash \boldsymbol{\Phi} \backslash \overrightarrow{\bar{\Lambda}}(\text { see }(4.3-12)) \\
& \square_{n} \mathbf{H} \stackrel{\text { def }}{=} \partial_{n} \mathbf{H}+i\left(\overleftarrow{\Gamma}_{n} \mathbf{H}-\mathbf{H} \overrightarrow{\boldsymbol{\Gamma}}_{n}\right) ; \quad \mathbf{H} \stackrel{\text { def }}{=} \frac{1}{\sqrt{8}} m_{\mathbf{h}} \mathbf{\Phi} ; m_{\mathrm{h}} \stackrel{\text { def }}{=} \text { Higgs mass } \approx 125 \mathrm{GeV}
\end{aligned}
$$

II is the $8 \times 8$ identity matrix.

$k \stackrel{\text { def }}{=} \frac{3}{\sqrt{2}} \theta_{\mathrm{s}}($ Computed later on in $(4.5-33))$

To obtain the multi-generation Lagrangian that describes the interactions of all three generations of fermions, one has to simply replace the unified spinors field $\overleftrightarrow{\boldsymbol{\Psi}}$ with the mixed spinors field defined in (4.2-24) and use (4.2-25) to (4.2-27) accordingly.

We still have to repay our debt regarding the values of the various coupling constants appearing in (4.1-1), as well as the constant $k$, appearing in (4.4-10). We also want to compute all the gauge bosons' masses.

However, before we do all that, we need to establish the unified Lagrangian invariance under unitary transformations of the type presented in (4.2-6). 


\section{Unified Charge Vectors Theory}

To establish this invariance, we first want to use (4.2-10) to show that:

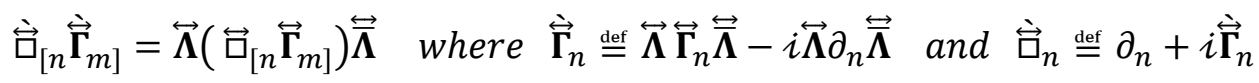

To prove this, we write:

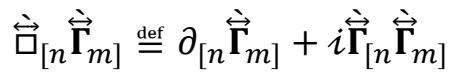

$$
\begin{aligned}
& =\partial_{[n}\left(\overleftrightarrow{\Lambda} \overleftrightarrow{\Gamma}_{m]} \stackrel{\leftrightarrow}{\Lambda}-i \overleftrightarrow{\Lambda} \partial_{m]} \stackrel{\leftrightarrow}{\Lambda}\right)+i\left(\overleftrightarrow{\Lambda} \overleftrightarrow{\Gamma}_{[n} \stackrel{\leftrightarrow}{\Lambda}-i \overleftrightarrow{\Lambda} \partial_{[n} \stackrel{\leftrightarrow}{\Lambda}\right)\left(\overleftrightarrow{\Lambda} \overleftrightarrow{\Gamma}_{m]} \overleftrightarrow{\bar{\Lambda}}-i \overleftrightarrow{\Lambda} \partial_{m]} \overleftrightarrow{\Lambda}\right)
\end{aligned}
$$

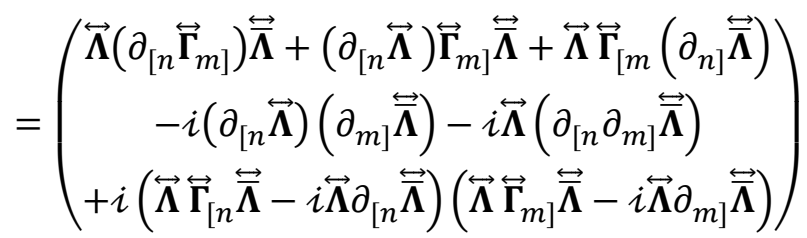

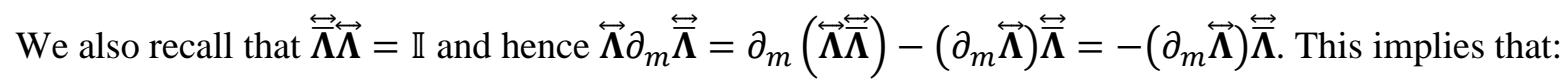

And hence:

$$
\overleftrightarrow{\Lambda} \overleftrightarrow{\Gamma}_{n} \overleftrightarrow{\Lambda}-i \overleftrightarrow{\Lambda} \partial_{n} \overleftrightarrow{\bar{\Lambda}}=\overleftrightarrow{\Lambda} \overleftrightarrow{\Gamma}_{n} \overleftrightarrow{\Lambda}+i\left(\partial_{n} \overleftrightarrow{\Lambda}\right) \overleftrightarrow{\Lambda}
$$

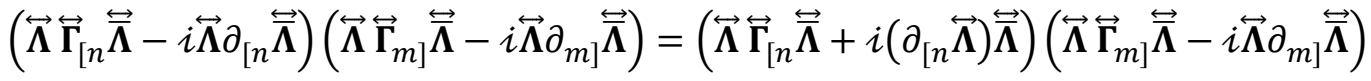

$$
\begin{aligned}
& =\overleftrightarrow{\Lambda} \overleftrightarrow{\boldsymbol{\Gamma}}_{[n} \overleftrightarrow{\boldsymbol{\Gamma}}_{m]} \overleftrightarrow{\boldsymbol{\Lambda}}+\left(\partial_{[n} \overleftrightarrow{\boldsymbol{\Lambda}}\right)\left(\partial_{m]} \stackrel{\leftrightarrow}{\boldsymbol{\Lambda}}\right)-i\left(\overleftrightarrow{\Lambda} \overleftrightarrow{\boldsymbol{\Gamma}}_{[n} \partial_{m]} \overleftrightarrow{\mathbf{\Lambda}}-\left(\partial_{[n} \overleftrightarrow{\Lambda}\right) \overleftrightarrow{\boldsymbol{\Gamma}}_{m]} \overleftrightarrow{\mathbf{\Lambda}}\right)
\end{aligned}
$$

Plugging (4.4-14) in (4.4-12), we obtain:

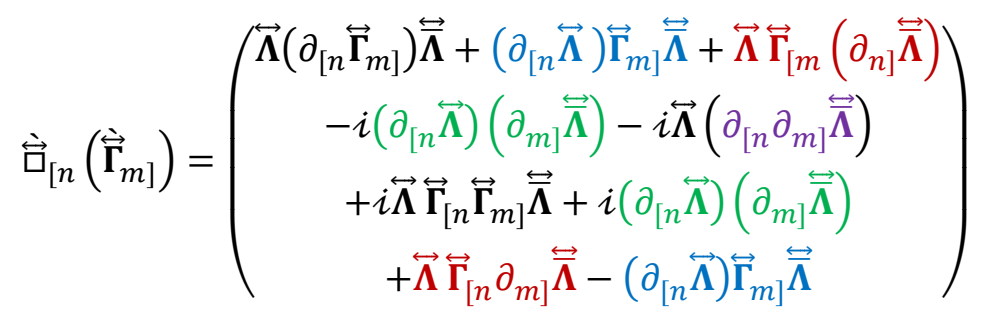

Since $\overleftrightarrow{\Lambda} \overleftrightarrow{\Gamma}_{[m}\left(\partial_{n]} \overleftrightarrow{\Lambda}\right)=-\overleftrightarrow{\Lambda} \overleftrightarrow{\Gamma}_{[n}\left(\partial_{m]} \overleftrightarrow{\Lambda}\right)$, and $\partial_{[n} \partial_{m]}=\partial_{n} \partial_{m}-\partial_{\mathrm{m}} \partial_{n}=0$, we conclude that all the colored terms in (4.4-15) cancel out, and we are left with:

$$
\overleftrightarrow{\mathrm{D}}_{[n} \overleftrightarrow{\mathrm{\Gamma}}_{m]}=\overleftrightarrow{\boldsymbol{\Lambda}}\left(\partial_{[n} \overleftrightarrow{\boldsymbol{\Gamma}}_{m]}+i \overleftrightarrow{\boldsymbol{\Gamma}}_{[n} \overleftrightarrow{\boldsymbol{\Gamma}}_{m]}\right) \overleftrightarrow{\bar{\Lambda}}=\overleftrightarrow{\boldsymbol{\Lambda}}\left(\overleftrightarrow{口}_{[n} \overleftrightarrow{\boldsymbol{\Gamma}}_{m]}\right) \overleftrightarrow{\mathbf{\Lambda}}
$$

We also recall that by (4.2-9), (4.3-6), and (4.3-18), we have:

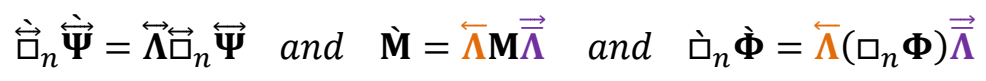




\section{Unified Charge Vectors Theory}

We now define the transformed Lagrangian $\grave{\mathcal{L}}$ as follows:

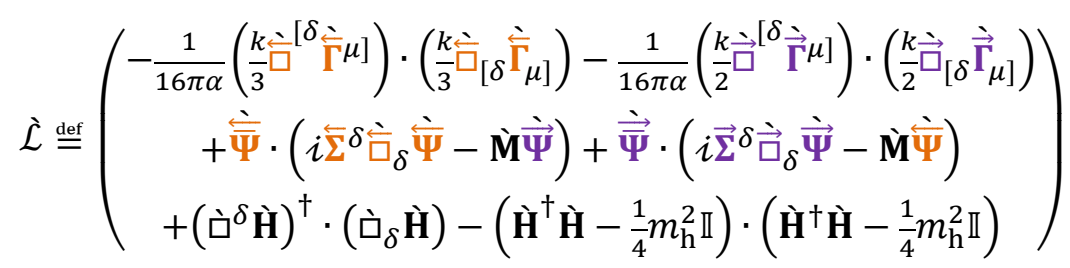

Using (4.4-16), and the fact that $\mathbf{A} \cdot \mathbf{B} \stackrel{\text { def }}{=} \operatorname{Trace}(\mathbf{A B})=\operatorname{Trace}(\mathbf{B A})=\mathbf{B} \cdot \mathbf{A}$, we conclude that:

And likewise:

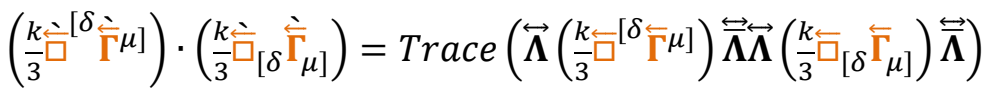

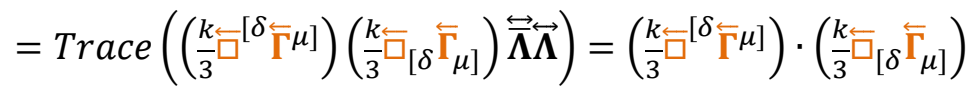

$$
\left(\frac{k}{2} \dot{\vec{\square}}^{[\delta} \dot{\vec{\Gamma}}^{\mu]}\right) \cdot\left(\frac{k}{2} \dot{\vec{\square}}_{[\delta} \dot{\vec{\Gamma}}_{\mu]}\right)=\left(\frac{k}{2} \vec{\square}^{[\delta} \vec{\Gamma}^{\mu]}\right) \cdot\left(\frac{k}{2} \vec{\square}_{[\delta} \vec{\Gamma}_{\mu]}\right)
$$

Using (4.4-17), we see that:

And likewise:

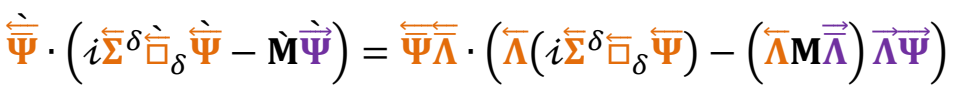

$$
\begin{aligned}
& =\overleftarrow{\overleftarrow{\Psi}} \cdot\left(\overleftarrow{\Lambda}\left(i \overleftarrow{\Sigma} \delta_{\square_{\delta}} \overleftarrow{\Psi}\right)-\mathbf{M} \vec{\Psi}\right)
\end{aligned}
$$

$$
\dot{\vec{\Psi}} \cdot\left(i \vec{\Sigma} \delta \dot{\vec{\square}}_{\delta} \dot{\vec{\Psi}}-\grave{\mathbf{M}} \dot{\Psi}\right)=\overrightarrow{\vec{\Psi}} \cdot\left(i \vec{\Sigma} \delta \vec{\square}_{\delta} \vec{\Psi}-\mathbf{M} \Psi\right)
$$

Finally, recalling that by (4.4-8) we have $\mathbf{H} \stackrel{\text { def }}{=} \frac{1}{\sqrt{8}} m_{\mathrm{h}} \boldsymbol{\Phi}$, and by (4.4-17) $\dot{\mathrm{d}}_{n} \grave{\boldsymbol{\Phi}}=\overleftarrow{\Lambda}\left(\square_{n} \boldsymbol{\Phi}\right) \vec{\Lambda}$, we obtain:

$$
\begin{aligned}
& \left(\grave{\square}^{\delta} \grave{\mathbf{H}}\right)^{\dagger} \cdot\left(\grave{\square}_{\delta} \grave{\mathbf{H}}\right)-\left(\grave{\mathbf{H}}^{\dagger} \grave{\mathbf{H}}-\frac{1}{4} m_{\mathrm{h}}^{2} \mathbb{I}\right) \cdot\left(\grave{\mathbf{H}}^{\dagger} \grave{\mathbf{H}}-\frac{1}{4} m_{\mathrm{h}}^{2} \mathbb{I}\right) \\
= & \left(\square^{\delta} \mathbf{H}\right)^{\dagger} \cdot\left(\square_{\delta} \mathbf{H}\right)-\left(\mathbf{H}^{\dagger} \mathbf{H}-\frac{1}{4} m_{\mathrm{h}}^{2} \mathbb{I}\right) \cdot\left(\mathbf{H}^{\dagger} \mathbf{H}-\frac{1}{4} m_{\mathrm{h}}^{2} \mathbb{I}\right)
\end{aligned}
$$

Substituting all these results back in (4.4-18), we conclude that indeed:

$$
\grave{\mathcal{L}}=\mathcal{L}
$$

Hence, we have managed to show that the unified Lagrangian is invariant under all $\mathrm{U}(8)$ transformations. 


\section{Unified Charge Vectors Theory}

\subsection{The Coupling Constants}

Our next task is to compute the coupling coefficients $\theta, k, \overleftrightarrow{k}_{1}, k_{2}, \overleftrightarrow{k}_{3}, \overleftrightarrow{k}_{4}$. The values of these constants can be deduced from the form the Lagrangian must obtain. Specifically, quantum field theory tells us that the Lagrangian of any Chargeless Gauge Field $\mathrm{P}_{n}$ with mass $m$ must have the form:

$$
\mathcal{L}=-\frac{1}{4}\left(\partial^{[\delta} \mathrm{P}^{\mu]}\right)\left(\partial_{[\delta} \mathrm{P}_{\mu]}\right)+\frac{1}{2} m^{2} \mathrm{P}_{\delta} \mathrm{P}^{\delta} \quad \text { Where } \quad \partial_{[\mathrm{i}} \mathrm{P}_{\mathrm{j}]} \stackrel{\text { def }}{=} \partial_{\mathrm{i}} \mathrm{P}_{\mathrm{j}}-\partial_{\mathrm{j}} \mathrm{P}_{\mathrm{i}}
$$

Notice that since this field represents a chargeless particle, it has no interaction terms. On the other hand, if the particle is charged, then it can interact with some external potential field $\mathrm{P}_{n}$, and we now have to promote the field to a complex gauge field, denoted by $\mathrm{X}_{n}$, and use the following Proca Lagrangian:

$$
\mathcal{L}=-\frac{1}{2}\left(\partial^{[\delta} \mathrm{X}^{\mu]}+i k_{p} \mathrm{P}^{[\delta} \mathrm{X}^{\mu]}\right)^{*}\left(\partial_{[\delta} \mathrm{X}_{\mu]}+i k_{p} \mathrm{P}_{[\delta} \mathrm{X}_{\mu]}\right)+m^{2} \mathrm{X}_{\delta}^{*} \mathrm{X}^{\delta}
$$

Where $k_{p}$ is some coupling coefficient.

Now, the bosons periodic table (3.3-7) tells us that potential bosons occupying the diagonal cells are all chargeless since they are the product of the annihilation of a particle and its anti-particle, and hence their unified charge vectors vanish. Therefore, we conclude that the field equations for the potential fields $\mathrm{B}_{n}, \mathrm{~W}_{n}, \mathrm{G}_{n}^{\perp}, \mathrm{G}_{n}^{\vdash}$ must all be of the form (4.5-1).

On the other hand, all the off-diagonal bosons in the bosons periodic table are charged, i.e., their unified charge vectors do not vanish. Hence, we conclude that the Lagrangian terms of all these fields must be of the form (4.5-2).

To make sure that this is indeed the case, we need to expand the following term, appearing in the unified Lagrangian (4.4-1):

$$
-\frac{1}{16 \pi \alpha}\left(\frac{k \overleftarrow{\square}}{3}\left[\delta \overleftarrow{\Gamma}^{\mu]}\right) \cdot\left(\frac{k}{3} \overleftarrow{\square}_{[\delta} \overleftarrow{\Gamma}_{\mu]}\right)-\frac{1}{16 \pi \alpha}\left(\frac{k}{2} \vec{\square}^{[\delta} \vec{\Gamma}^{\mu]}\right) \cdot\left(\frac{k}{2} \vec{\square}_{[\delta} \vec{\Gamma}_{\mu]}\right)\right.
$$

Or equivalently:

$$
-\frac{1}{4}\left(\frac{\left.k \overleftarrow{\square}^{[\delta \overleftarrow{\Gamma}} \mu\right]}{3 \sqrt{4 \pi \alpha}}\right) \cdot\left(\frac{k \overleftarrow{\square}_{[\delta} \overleftarrow{\Gamma}_{\mu]}}{3 \sqrt{4 \pi \alpha}}\right)-\frac{1}{4}\left(\frac{k \vec{\square}^{[\delta} \vec{\Gamma}^{\mu]}}{2 \sqrt{4 \pi \alpha}}\right) \cdot\left(\frac{k \vec{\square}_{[\delta} \vec{\Gamma}_{\mu]}}{2 \sqrt{4 \pi \alpha}}\right)
$$

In fact, to ensure (4.5-1) and (4.5-2), we can safely ignore the interaction terms and focus our attention on the following simplified expression:

$$
-\frac{1}{4}\left(\frac{k \partial^{[\delta \overleftarrow{\Gamma} \mu]}}{3 \sqrt{4 \pi \alpha}}\right) \cdot\left(\frac{k \partial_{[\delta} \overleftarrow{\Gamma}_{\mu]}}{3 \sqrt{4 \pi \alpha}}\right)-\frac{1}{4}\left(\frac{k \partial^{\left[\delta \vec{\Gamma}^{\mu]}\right.}}{2 \sqrt{4 \pi \alpha}}\right) \cdot\left(\frac{k \partial_{[\delta} \vec{\Gamma}_{\mu]}}{2 \sqrt{4 \pi \alpha}}\right)
$$




\section{Unified Charge Vectors Theory}

So, our task is to show that when we express (4.5-5) in terms of its constituent fields, we get for the potential bosons the following terms:

$$
-\frac{1}{4}\left(\begin{array}{r}
\left(\partial^{[\delta} \mathrm{B}^{\mu]}\right)\left(\partial_{[\delta} \mathrm{B}_{\mu]}\right)+\left(\partial^{[\delta} \mathrm{W}^{\mu]}\right)\left(\partial_{[\delta} \mathrm{W}_{\mu]}\right) \\
+\left(\partial^{[\delta} \mathrm{G}_{\perp}^{\mu]}\right)\left(\partial_{[\delta} \mathrm{G}_{\mu]}^{\perp}\right)+\left(\partial^{[\delta} \mathrm{G}_{\vdash}^{\mu]}\right)\left(\partial_{[\delta} \mathrm{G}_{\mu]}^{\vdash}\right)
\end{array}\right)
$$

And for the charged bosons:

$$
-\frac{1}{2}\left(\begin{array}{c}
\sum_{x, y}\left(\partial^{[\delta} \mathrm{G}_{\overline{\mathrm{x} y}}^{\mu]}\right)\left(\partial_{[\delta} \mathrm{G}_{\mu]}^{\overline{\mathrm{y} x}}\right)+\sum_{x, y}\left(\partial^{[\delta} \mathrm{W}_{-\overline{\mathrm{x} y}}^{\mu]}\right)\left(\partial_{[\delta} \mathrm{W}_{\mu]}^{+\overline{\mathrm{x} y}}\right)+\left(\partial^{[\delta} \overleftrightarrow{\mathrm{W}}_{-}^{\mu]}\right)\left(\partial_{[\delta} \overleftrightarrow{\mathrm{W}}_{\mu]}^{+}\right) \\
+\sum_{c}\left(\left(\partial^{[\delta} \mathrm{W}_{-\mathrm{c}}^{\mu]}\right)\left(\partial_{[\delta} \mathrm{W}_{\mu]}^{+\overline{\mathrm{c}}}\right)+\left(\partial^{[\delta} \mathrm{W}_{-\overline{\mathrm{v}} \mathrm{c}}^{\mu]}\right)\left(\partial_{[\delta} \mathrm{W}_{\mu]}^{+\overline{\mathrm{c}} \nu}\right)+\left(\partial^{[\delta} \mathrm{W}_{-\overline{\mathrm{e}} \mathrm{c}}^{\mu]}\right)\left(\partial_{[\delta} \mathrm{W}_{\mu]}^{+\overline{\mathrm{c} e}}\right)\right)
\end{array}\right)
$$

To proceed with this computation, we recall that by (4.1-8) and (4.1-9), we have:

And:

$$
\frac{\overleftarrow{\Gamma}_{n}}{\sqrt{4 \pi \alpha}}=\frac{\mathrm{B}_{n}}{2 \theta_{\mathrm{c}}}\left[\begin{array}{ll}
\mathfrak{T} & 0 \\
0 & \mathfrak{T}
\end{array}\right]+\left[\begin{array}{cc}
\mathbf{G}_{n} & 0 \\
0 & \mathbf{G}_{n}
\end{array}\right]+\frac{\mathbf{W}_{n}}{2 \theta_{\mathbf{s}}}\left[\begin{array}{cc}
\mathbf{I} & 0 \\
0 & -\mathbf{I}
\end{array}\right]+\left[\begin{array}{ll}
\overleftarrow{\mathbf{W}}_{n} & \overleftarrow{\widetilde{W}}_{n}^{+} \\
\overleftarrow{\widetilde{\mathbf{W}}}_{n}^{-} & \overleftarrow{\mathbf{W}}_{n}
\end{array}\right]
$$

$$
\frac{\vec{\Gamma}_{n}}{\sqrt{4 \pi \alpha}}=\frac{\mathrm{B}_{n}}{2 \theta_{c}}\left[\begin{array}{cc}
\mathfrak{T} & 0 \\
0 & \mathfrak{T}
\end{array}\right]+\left[\begin{array}{ll}
\mathbf{G}_{n} & 0 \\
0 & \mathbf{G}_{n}
\end{array}\right]+\frac{\mathrm{B}_{n}}{2 \theta_{\mathrm{c}}}\left[\begin{array}{rr}
\mathbf{I} & 0 \\
0 & -\mathbf{I}
\end{array}\right]+\left[\begin{array}{ll}
\overrightarrow{\widetilde{\mathbf{W}}}_{n} & \overrightarrow{\widetilde{\mathbf{W}}}_{n}^{+} \\
\overrightarrow{\widetilde{\mathbf{W}}}_{n}^{-} & \overrightarrow{\widetilde{\mathbf{W}}}_{n}
\end{array}\right]
$$

Where by $(4.1-10)$ :

$$
\mathfrak{T} \stackrel{\text { def }}{=}\left[\begin{array}{cccc}
-1 & 0 & 0 & 0 \\
0 & \frac{1}{3} & 0 & 0 \\
0 & 0 & \frac{1}{3} & 0 \\
0 & 0 & 0 & \frac{1}{3}
\end{array}\right] \quad \text { and } \quad \mathbf{I} \stackrel{\text { def }}{=}\left[\begin{array}{llll}
1 & 0 & 0 & 0 \\
0 & 1 & 0 & 0 \\
0 & 0 & 1 & 0 \\
0 & 0 & 0 & 1
\end{array}\right]
$$

And by (4.1-6) and (4.1-7):

$$
\begin{aligned}
& \mathbf{G}_{n} \stackrel{\text { def }}{=}\left[\begin{array}{cccc}
0 & 0 & 0 & 0 \\
0 & \frac{2 \sqrt{2}}{3} \mathbf{S}_{n}^{\mathrm{r}} & k_{2} \mathbf{G}_{n}^{\overline{\mathrm{g}} \mathrm{r}} & k_{2} \mathbf{G}_{n}^{\overline{\mathrm{br}}} \\
0 & k_{2} \mathbf{G}_{n}^{\overline{\mathrm{rg}}} & \frac{2 \sqrt{2}}{3} \mathbf{S}_{n}^{\mathrm{g}} & k_{2} \mathbf{G}_{n}^{\overline{\mathrm{g} g}} \\
0 & k_{2} \mathbf{G}_{n}^{\overline{\mathrm{rb}}} & k_{2} \mathbf{G}_{n}^{\overline{\mathrm{gb}}} & \frac{2 \sqrt{2}}{3} \mathbf{S}_{n}^{\mathrm{b}}
\end{array}\right]=\left[\begin{array}{cccc}
0 & 0 & 0 & 0 \\
0 & \frac{2 \sqrt{2}}{3} \mathbf{G}_{n}^{\perp} & k_{2} \mathbf{G}_{n}^{\overline{\mathrm{g}} \mathbf{r}} & k_{2} \mathbf{G}_{n}^{\overline{\mathrm{b}} \mathrm{r}} \\
0 & k_{2} \mathbf{G}_{n}^{\overline{\mathrm{rg}}} & -\frac{\sqrt{2}}{3}\left(\sqrt{3} \mathbf{G}_{n}^{+}+\mathbf{G}_{n}^{\perp}\right) & k_{2} \mathbf{G}_{n}^{\overline{\mathrm{b} g}} \\
0 & k_{2} \mathbf{G}_{n}^{\overline{\mathrm{rb}}} & k_{2} \mathbf{G}_{n}^{\overline{\mathrm{gb}}} & \frac{\sqrt{2}}{3}\left(\sqrt{3} \mathbf{G}_{n}^{+}-\mathbf{G}_{n}^{\perp}\right)
\end{array}\right]
\end{aligned}
$$

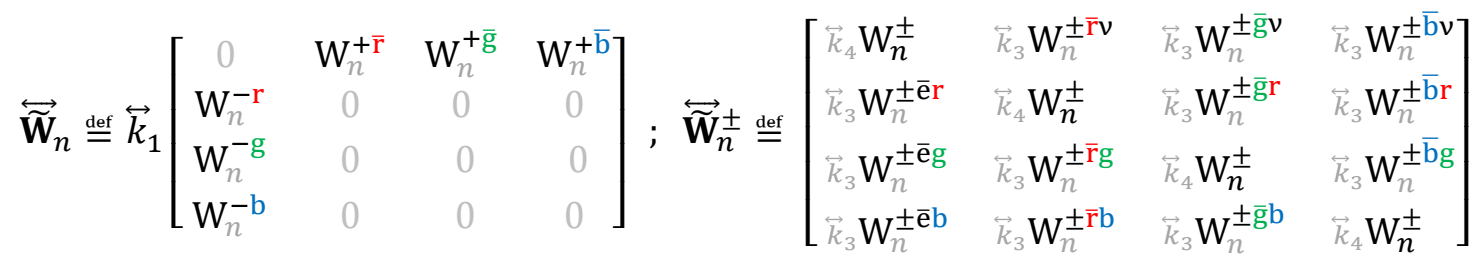




\section{Unified Charge Vectors Theory}

It also proves enormously helpful to realize the following useful identities:

$$
\begin{gathered}
\mathfrak{T} \cdot \mathbf{I} \stackrel{\text { def }}{=} \operatorname{Trace}(\mathfrak{I} \mathbf{I})=\operatorname{Trace}(\mathfrak{I})=0 \\
\mathfrak{T} \cdot \mathfrak{T}=\operatorname{Trace}(\mathfrak{T} \mathfrak{T})=1+\frac{1}{9}+\frac{1}{9}+\frac{1}{9}=\frac{4}{3} \\
\mathbf{I} \cdot \mathbf{I}=\operatorname{Trace}(\mathbf{I})=4 \\
\mathfrak{T} \cdot \mathbf{G}_{n}=\mathbf{I} \cdot \mathbf{G}_{n}=\mathfrak{T} \cdot \overleftrightarrow{\widetilde{\mathbf{W}}}_{n}=\mathbf{I} \cdot \overleftrightarrow{\mathbf{W}}_{n}=\mathbf{G}_{n} \cdot \overleftrightarrow{\mathbf{W}}_{m}=0
\end{gathered}
$$

In other words, we can say that the matrices $\mathfrak{T}, \mathbf{I}, \mathbf{G}_{n}$, and $\overleftrightarrow{\mathbf{W}}_{m}$, are orthogonal to each other (i.e., their dot product vanishes). These orthogonality identities prevent the different fields from "clashing" with each other when we perform the dot products in (4.5-5). Hence, we can focus on the following surviving terms:

For the B field, we have:

$$
\left(\frac{\partial^{[\delta} \mathrm{B}^{\mu]}}{2 \theta_{\mathrm{c}}}\left[\begin{array}{cc}
\mathfrak{T} & 0 \\
0 & \mathfrak{T}
\end{array}\right]\right) \cdot\left(\frac{\partial_{[\delta} \mathrm{B}_{\mu]}}{2 \theta_{\mathrm{c}}}\left[\begin{array}{cc}
\mathfrak{T} & 0 \\
0 & \mathfrak{T}
\end{array}\right]\right)=\frac{2}{3 \theta_{\mathrm{c}}^{2}}\left(\partial^{\left[\delta \mathrm{B}^{\mu]}\right.}\right)\left(\partial_{[\delta} \mathrm{B}_{\mu]}\right)
$$

And:

$$
\left(\frac{\partial^{[\delta} \mathrm{B}^{\mu]}}{2 \theta_{\mathrm{c}}}\left[\begin{array}{cc}
\mathbf{I} & 0 \\
0 & -\mathbf{I}
\end{array}\right]\right) \cdot\left(\frac{\partial_{[\delta} \mathrm{B}_{\mu]}}{2 \theta_{\mathrm{c}}}\left[\begin{array}{cc}
\mathbf{I} & 0 \\
0 & -\mathbf{I}
\end{array}\right]\right)=\frac{6}{3 \theta_{\mathrm{c}}^{2}}\left(\partial^{[\delta} \mathrm{B}^{\mu]}\right)\left(\partial_{[\delta} \mathrm{B}_{\mu]}\right)
$$

And for the gluons:

$$
\begin{gathered}
{\left[\begin{array}{cc}
\partial^{[\delta} \mathbf{G}^{\mu]} & 0 \\
0 & \partial^{[\delta} \mathbf{G}^{\mu]}
\end{array}\right] \cdot\left[\begin{array}{cc}
\partial_{[\delta} \mathbf{G}_{\mu]} & 0 \\
0 & \partial_{[\delta} \mathbf{G}_{\mu]}
\end{array}\right]=2\left(\partial^{[\delta} \mathbf{G}^{\mu]}\right) \cdot\left(\partial_{[\delta} \mathbf{G}_{\mu]}\right)} \\
=\frac{8}{3}\left(\partial^{[\delta} \mathrm{G}_{\perp}^{\mu]}\right)\left(\partial_{[\delta} \mathrm{G}_{\mu]}^{\perp}\right)+\frac{8}{3}\left(\partial^{[\delta} \mathrm{G}_{\vdash}^{\mu]}\right)\left(\partial_{[\delta} \mathrm{G}_{\mu]}^{\vdash}\right)+4\left(k_{2}\right)^{2} \sum_{x, y}\left(\partial^{[\delta} \mathrm{G}_{\overline{\mathrm{x}} \mathrm{y}}^{\mu]}\right)\left(\partial_{[\delta} \mathrm{G}_{\mu]}^{\overline{\mathrm{y} x}}\right)
\end{gathered}
$$

For the $\mathrm{W}_{n}$ fields, we have:

$$
\left(\frac{\partial^{[\delta} \mathrm{W}^{\mu]}}{2 \theta_{\mathrm{S}}}\left[\begin{array}{rr}
\mathbf{I} & 0 \\
0 & -\mathbf{I}
\end{array}\right]\right) \cdot\left(\frac{\partial_{[\delta} \mathrm{W}_{\mu]}}{2 \theta_{\mathrm{S}}}\left[\begin{array}{rr}
\mathbf{I} & 0 \\
0 & -\mathbf{I}
\end{array}\right]\right)=\frac{2}{\theta_{\mathrm{S}}^{2}}\left(\partial^{[\delta} \mathrm{W}^{\mu]}\right)\left(\partial_{[\delta} \mathrm{W}_{\mu]}\right)
$$

And:

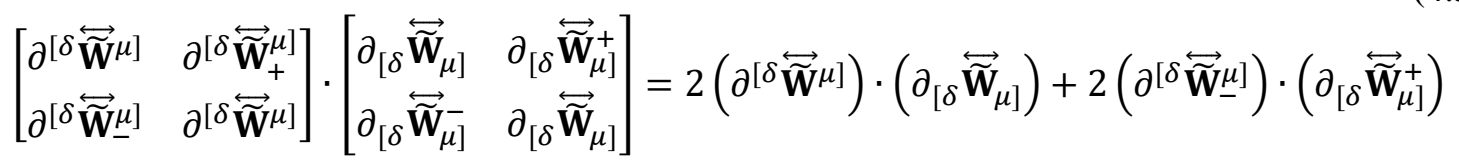

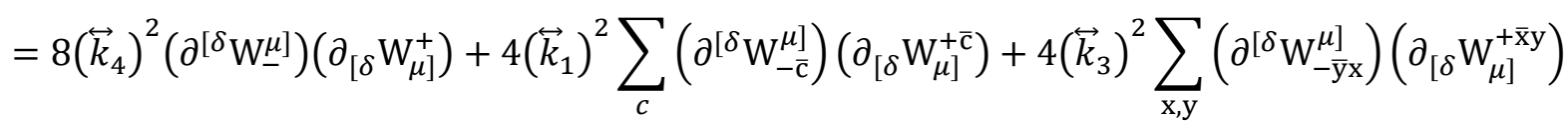




\section{Unified Charge Vectors Theory}

Putting it all together, we conclude that:

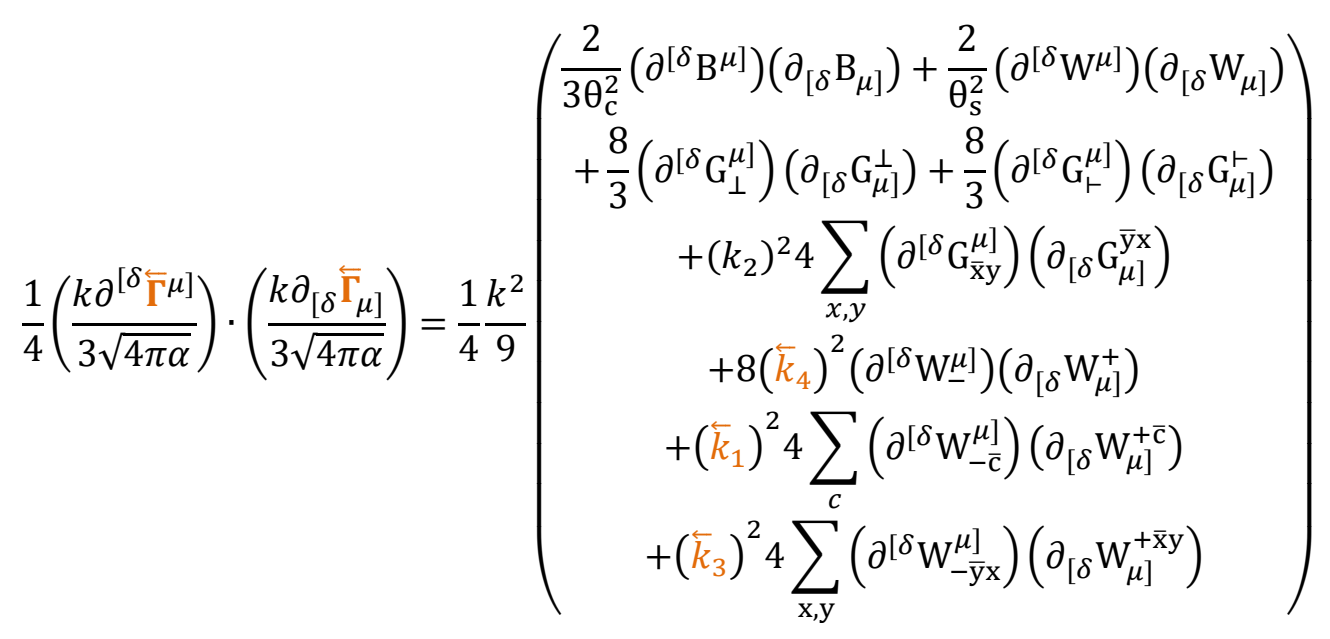

And:

$$
\frac{1}{4}\left(\frac{k \partial^{\left[\delta \vec{\Gamma}^{\mu]}\right.}}{2 \sqrt{4 \pi \alpha}}\right) \cdot\left(\frac{k \partial_{[\delta} \vec{\Gamma}_{\mu]}}{2 \sqrt{4 \pi \alpha}}\right)=\frac{1}{4} \frac{k^{2}}{4}\left(\begin{array}{c}
\frac{8}{3 \theta_{\mathrm{c}}^{2}}\left(\partial^{[\delta} \mathrm{B}^{\mu]}\right)\left(\partial_{[\delta} \mathrm{B}_{\mu]}\right) \\
+\frac{8}{3}\left(\partial^{[\delta} \mathrm{G}_{\perp}^{\mu]}\right)\left(\partial_{[\delta} \mathrm{G}_{\mu]}^{\perp}\right)+\frac{8}{3}\left(\partial^{[\delta} \mathrm{G}_{\vdash}^{\mu]}\right)\left(\partial_{[\delta} \mathrm{G}_{\mu]}^{\vdash}\right) \\
+\left(k_{2}\right)^{2} 4 \sum_{x, y}\left(\partial^{[\delta} \mathrm{G}_{\overline{\mathrm{x}} \mathrm{y}}^{\mu]}\right)\left(\partial_{[\delta} \mathrm{G}_{\mu]}^{\overline{\mathrm{y} x}}\right) \\
+8\left(\vec{k}_{4}\right)^{2}\left(\partial^{[\delta} \mathrm{W}_{-}^{\mu]}\right)\left(\partial_{[\delta} \mathrm{W}_{\mu]}^{+}\right) \\
+\left(\vec{k}_{1}\right)^{2} 4 \sum_{c}\left(\partial^{[\delta} \mathrm{W}_{-\overline{\mathrm{c}}}^{\mu]}\right)\left(\partial_{[\delta} \mathrm{W}_{\mu]}^{+\overline{\mathrm{c}}}\right) \\
+\left(\vec{k}_{3}\right)^{2} 4 \sum_{\mathrm{x}, \mathrm{y}}\left(\partial^{[\delta} \mathrm{W}_{-\overline{\mathrm{y}} \mathrm{x}}^{\mu]}\right)\left(\partial_{[\delta} \mathrm{W}_{\mu]}^{+\overline{\mathrm{x}} \mathrm{y}}\right)
\end{array}\right)
$$

So, for the chargeless fields $\mathrm{B}_{n}, \mathrm{~W}_{n}, \mathrm{G}_{n}^{\perp}$, and $\mathrm{G}_{n}^{\vdash}$, we must have:

And

$$
\frac{1}{4}\left(\frac{k^{2}}{9} \frac{2}{3 \theta_{\mathrm{c}}^{2}}+\frac{k^{2}}{4} \frac{8}{3 \theta_{\mathrm{c}}^{2}}\right)\left(\partial^{[\delta} \mathrm{B}^{\mu]}\right)\left(\partial_{[\delta} \mathrm{B}_{\mu]}\right)=\frac{1}{4}\left(\partial^{[\delta} \mathrm{B}^{\mu]}\right)\left(\partial_{[\delta} \mathrm{B}_{\mu]}\right)
$$

And

$$
\frac{1}{4}\left(\frac{k^{2}}{9} \frac{2}{\theta_{\mathrm{s}}^{2}} \quad\right)\left(\partial^{[\delta} \mathrm{W}^{\mu]}\right)\left(\partial_{[\delta} \mathrm{W}_{\mu]}\right)=\frac{1}{4}\left(\partial^{[\delta} \mathrm{W}^{\mu]}\right)\left(\partial_{[\delta} \mathrm{W}_{\mu]}\right)
$$

$$
\frac{1}{4}\left(\frac{k^{2}}{9}+\frac{k^{2}}{4}\right) \frac{8}{3}\left(\partial^{[\delta} \mathrm{G}_{\perp}^{\mu]}\right)\left(\partial_{[\delta} \mathrm{G}_{\mu]}^{\perp}\right)=\frac{1}{4}\left(\partial^{[\delta} \mathrm{G}_{\perp}^{\mu]}\right)\left(\partial_{[\delta} \mathrm{G}_{\mu]}^{\perp}\right)
$$

And

$$
\frac{1}{4}\left(\frac{k^{2}}{9}+\frac{k^{2}}{4}\right) \frac{8}{3}\left(\partial^{[\delta} \mathrm{G}_{\vdash}^{\mu]}\right)\left(\partial_{[\delta} \mathrm{G}_{\mu]}^{\vdash}\right)=\frac{1}{4}\left(\partial^{[\delta} \mathrm{G}_{\vdash}^{\mu]}\right)\left(\partial_{[\delta} \mathrm{G}_{\mu]}^{\vdash}\right)
$$




\section{Unified Charge Vectors Theory}

Likewise, for the charged fields $\mathrm{G}_{n}^{\overline{\mathrm{x}} \mathrm{y}}, \mathrm{W}_{n}^{+}, \mathrm{W}_{n}^{+\overline{\mathrm{c}}}$, and $\mathrm{W}_{n}^{+\overline{\mathrm{x}} \mathrm{y}}$, we must have:

$$
\frac{1}{4}\left(\frac{k^{2}}{9}+\frac{k^{2}}{4}\right)\left(k_{2}\right)^{2} 4\left(\partial^{[\delta} \mathrm{G}_{\overline{\mathrm{x}} \mathrm{y}}^{\mu]}\right)\left(\partial_{[\delta} \mathrm{G}_{\mu]}^{\overline{\mathrm{y}} \mathrm{x}}\right)=\frac{1}{2}\left(\partial^{[\delta} \mathrm{G}_{\overline{\mathrm{x}} \mathrm{y}}^{\mu]}\right)\left(\partial_{[\delta} \mathrm{G}_{\mu]}^{\overline{\mathrm{y}} \mathrm{x}}\right)
$$

And

$$
\frac{1}{4}\left(\frac{k^{2}}{9}\left(\overleftarrow{k}_{4}\right)^{2}+\frac{k^{2}}{4}\left(\vec{k}_{4}\right)^{2}\right) 8\left(\partial^{[\delta} \mathrm{W}_{-}^{\mu]}\right)\left(\partial_{[\delta} \mathrm{W}_{\mu]}^{+}\right)=\frac{1}{2}\left(\partial^{[\delta} \mathrm{W}_{-}^{\mu]}\right)\left(\partial_{[\delta} \mathrm{W}_{\mu]}^{+}\right)
$$

And

$$
\frac{1}{4}\left(\frac{k^{2}}{9}\left(\overleftarrow{k}_{1}\right)^{2}+\frac{k^{2}}{4}\left(\vec{k}_{1}\right)^{2}\right) 4\left(\partial^{[\delta} \mathrm{W}_{-\bar{c}}^{\mu]}\right)\left(\partial_{[\delta} \mathrm{W}_{\mu]}^{+\overline{\mathrm{c}}}\right)=\frac{1}{2}\left(\partial^{[\delta} \mathrm{W}_{-\overline{\mathrm{c}}}^{\mu]}\right)\left(\partial_{[\delta} \mathrm{W}_{\mu]}^{+\overline{\mathrm{c}}}\right)
$$

And

$$
\frac{1}{4}\left(\frac{k^{2}}{9}\left(\overleftarrow{k}_{3}\right)^{2}+\frac{k^{2}}{4}\left(\vec{k}_{3}\right)^{2}\right) 4\left(\partial^{[\delta} \mathrm{W}_{-\overline{\mathrm{y}} \mathrm{x}}^{\mu]}\right)\left(\partial_{[\delta} \mathrm{W}_{\mu]}^{+\overline{\mathrm{x}} \mathrm{y}}\right)=\frac{1}{2}\left(\partial^{[\delta} \mathrm{W}_{-\overline{\mathrm{y}} \mathrm{x}}^{\mu]}\right)\left(\partial_{[\delta} \mathrm{W}_{\mu]}^{+\overline{\mathrm{x}} \mathrm{y}}\right)
$$

From (4.5-24), we conclude that:

$$
\frac{1}{4}\left(\frac{k^{2}}{9} \frac{2}{3 \theta_{\mathrm{c}}^{2}}+\frac{k^{2}}{4} \frac{8}{3 \theta_{\mathrm{c}}^{2}}\right)=\frac{1}{4} \quad \text { so } k^{2}=\frac{27}{20} \theta_{\mathrm{c}}^{2}=\frac{27}{20}\left(1-\theta_{\mathrm{s}}^{2}\right)
$$

From (4.5-25), we conclude that:

$$
\frac{1}{4} \frac{k^{2}}{9} \frac{2}{\theta_{\mathrm{s}}^{2}}=\frac{1}{4} \quad \text { so } \quad k^{2}=\frac{9}{2} \theta_{\mathrm{s}}^{2} \text { and hence } k=\frac{3}{\sqrt{2}} \theta_{\mathrm{s}}
$$

Solving (4.5-32) and (4.5-33) for $\theta_{\mathrm{s}}^{2}$, we conclude that:

$$
\frac{27}{20}\left(1-\theta_{\mathrm{s}}^{2}\right)=\frac{9}{2} \theta_{\mathrm{s}}^{2} \text { so, } \quad \theta_{\mathrm{s}}^{2} \stackrel{\text { def }}{=} \sin ^{2} \theta=\frac{3}{13} \approx 0.23077 \approx 0.23105(1-0.0012)
$$

This is a genuine moment of eureka! Given that the experimentally measured value of $\sin ^{2} \theta$ at $91.2 \mathrm{GeV} / \mathrm{c}$ is $0.23120 \pm 0.00015$, we see that our prediction is only $0.12 \%$ lower than the lowest measured value, which makes perfect sense since $\sin ^{2} \theta$ decreases as energy increases.

Plugging this result back in (4.5-33), we conclude that:

$$
k^{2}=\frac{9}{2} \theta_{\mathrm{s}}^{2}=\frac{27}{26}
$$




\section{Unified Charge Vectors Theory}

Continuing with (4.5-26) and (4.5-27), we conclude that:

$$
\frac{1}{4}\left(\frac{k^{2}}{9}+\frac{k^{2}}{4}\right) \frac{8}{3}=\frac{1}{4} \quad \text { so } \quad k^{2}=\frac{3}{8}\left(\frac{1}{9}+\frac{1}{4}\right)^{-1}=\frac{27}{26}
$$

The agreement between (4.5-35) and (4.5-36) is an important nontrivial confirmation of internal consistency.

From (4.5-28), we conclude that:

$$
\frac{1}{4}\left(\frac{k^{2}}{9}+\frac{k^{2}}{4}\right)\left(k_{2}\right)^{2} 4=\frac{1}{2} \quad \text { so } \quad k_{2}=\frac{1}{\sqrt{2 k^{2}\left(\frac{1}{9}+\frac{1}{4}\right)}}=\frac{2}{\sqrt{3}}
$$

Using (4.5-29), and the fact that experimentally $\vec{k}_{4}=0$, we conclude that:

$$
\frac{1}{4}\left(\frac{k^{2}}{9}\left(\overleftarrow{k}_{4}\right)^{2}+\frac{k^{2}}{4}\left(\vec{k}_{4}\right)^{2}\right) 8=\frac{1}{2} \quad \text { so } \quad \overleftarrow{k}_{4}=\frac{\sqrt{2}}{2 \theta_{\mathrm{s}}}
$$

Using (4.5-30), and speculating that $\vec{k}_{1}=0$, we conclude that:

$$
\frac{1}{4}\left(\frac{k^{2}}{9}\left(\overleftarrow{k}_{1}\right)^{2}+\frac{k^{2}}{4}\left(\vec{k}_{1}\right)^{2}\right) 4=\frac{1}{2} \text { so } \overleftarrow{k}_{1}=\frac{1}{\theta_{\mathrm{s}}}
$$

Using (4.5-31), and speculating that $\vec{k}_{3}=0$, we conclude that:

$$
\frac{1}{4}\left(\frac{k^{2}}{9}\left(\overleftarrow{k}_{3}\right)^{2}+\frac{k^{2}}{4}\left(\vec{k}_{3}\right)^{2}\right) 4=\frac{1}{2} \text { so } \quad \overleftarrow{k}_{3}=\frac{1}{\theta_{\mathrm{s}}}
$$




\section{Unified Charge Vectors Theory}

Now that we have worked out all the coupling constants, we can rewrite (4.5-11) and (4.5-12) as follows:

$$
\begin{aligned}
& \mathbf{G}_{n}=\sqrt{2}\left[\begin{array}{cccc}
0 & 0 & 0 & 0 \\
0 & \frac{\sqrt{2}}{\sqrt{3}}\left(\frac{2}{\sqrt{6}} G_{n}^{\perp}\right) & \frac{\sqrt{2}}{\sqrt{3}} G_{n}^{\overline{g r}} & \frac{\sqrt{2}}{\sqrt{3}} G_{n}^{\overline{b r}} \\
0 & \frac{\sqrt{2}}{\sqrt{3}} G_{n}^{\overline{\mathrm{r} g}} & -\frac{\sqrt{2}}{\sqrt{3}}\left(\frac{1}{\sqrt{2}} \mathbf{G}_{n}^{+}+\frac{1}{\sqrt{6}} G_{n}^{\perp}\right) & \frac{\sqrt{2}}{\sqrt{3}} G_{n}^{\overline{\mathrm{bg}}} \\
0 & \frac{\sqrt{2}}{\sqrt{3}} \mathrm{G}_{n}^{\overline{\mathrm{rb}}} & \frac{\sqrt{2}}{\sqrt{3}} \mathrm{G}_{n}^{\overline{\mathrm{gb}}} & \frac{\sqrt{2}}{\sqrt{3}}\left(\frac{1}{\sqrt{2}} \mathrm{G}_{n}^{-}-\frac{1}{\sqrt{6}} \mathrm{G}_{n}^{\perp}\right)
\end{array}\right]
\end{aligned}
$$

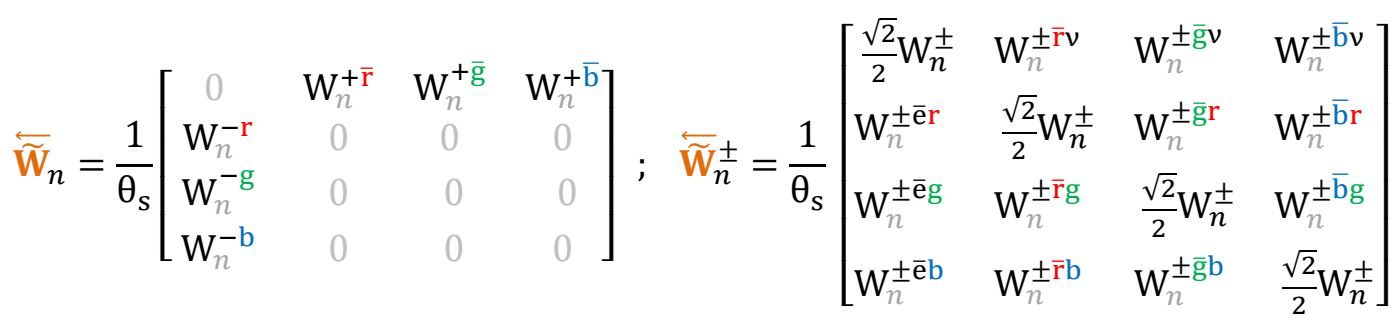

$$
\begin{aligned}
& \overrightarrow{\widetilde{\mathbf{W}}}_{n}=0 ; \quad \overrightarrow{\widetilde{\mathbf{W}}}_{n}^{ \pm}=0
\end{aligned}
$$

It is important to understand that (4.5-43) simply implies there exists a unitary transformation of the $\vec{\Gamma}_{n}$ fields in which the Higgs matrix obtains the diagonal form (4.3-10), and in which the $\overrightarrow{\mathbf{W}}_{n}$ and $\overrightarrow{\mathbf{W}}_{n}^{ \pm}$term vanish. As a geometric analogy, we can consider a flat space, for which there exists a coordinates-transformation in which the geometric connection field $\boldsymbol{\Gamma}$ vanishes everywhere. However, this is not the case in all coordinates-systems since $\Gamma$ does not transform like a tensor. The same applies to the $\overrightarrow{\widetilde{W}}_{n}$ and $\overrightarrow{\widetilde{W}}_{n}^{ \pm}$fields in $\vec{\Gamma}_{n}$. That is to say, while it can be made to vanish in the diagonal frame (4.3-10), it may not vanish in all other frames. However, this is perfectly fine since, as we have seen, the unified Lagrangian is invariant under all unitary transformations. 


\section{Unified Charge Vectors Theory}

\subsection{The Gauge Bosons Masses}

Next, we want to work out the masses of all the gauge bosons by expending the product $\left(\square^{\delta} \mathbf{H}\right)^{\dagger} \cdot\left(\square_{\delta} \mathbf{H}\right)$ appearing in (4.4-1).

By (4.4-8) we have $\mathbf{H} \stackrel{\text { def }}{=} \frac{1}{2} m_{\mathrm{h}} \boldsymbol{\Phi}$, so, when we transform to the diagonal form of the Higgs field, which according to (4.3-10) is given by $\mathbb{I} e^{\phi}$, we get:

$$
\mathbf{H}=\frac{1}{2} m_{\mathrm{h}} e^{\phi_{\mathbb{I}}}
$$

This, in turn, implies that:

$$
\begin{gathered}
\left(\square^{\delta} \mathbf{H}\right)^{\dagger} \cdot\left(\square_{\delta} \mathbf{H}\right)=m_{\mathrm{h}}^{2} \frac{1}{4}\left(\mathbb{I} \partial^{\delta} e^{\phi}-i e^{\phi}\left(\overleftarrow{\Gamma}^{\delta}-\vec{\Gamma}^{\delta}\right)\right) \cdot\left(\mathbb{I} \partial_{\delta} e^{\phi}+i e^{\phi}\left(\overleftarrow{\Gamma}_{\delta}-\vec{\Gamma}_{\delta}\right)\right) \\
=\frac{1}{4}(\mathbb{I} \cdot \mathbb{I})\left(m_{\mathrm{h}} \partial^{\delta} e^{\phi}\right)\left(m_{\mathrm{h}} \partial_{\delta} e^{\phi}\right)+\left(m_{\mathrm{h}} e^{\phi}\right)^{2} \frac{1}{4}\left(\overleftarrow{\Gamma}^{\delta}-\vec{\Gamma}^{\delta}\right) \cdot\left(\overleftarrow{\Gamma}_{\delta}-\vec{\Gamma}_{\delta}\right)
\end{gathered}
$$

We now define:

$$
\mathrm{h} \stackrel{\text { def }}{=} 2 m_{\mathrm{h}}\left(e^{\phi}-1\right) \quad \text { so } \quad m_{\mathrm{h}} e^{\phi}=m_{\mathrm{h}}+\frac{1}{2} \mathrm{~h}
$$

Plugging this back in (4.6-2) and using $\mathbb{I} \cdot \mathbb{I}=8$, we conclude that:

$$
\begin{gathered}
\left(\square^{\delta} \mathbf{H}\right)^{\dagger} \cdot\left(\square_{\delta} \mathbf{H}\right)=\frac{1}{2}\left(\partial^{\delta} \mathrm{h}\right)\left(\partial_{\delta} \mathrm{h}\right)+\left(m_{\mathrm{h}}^{2}+m_{\mathrm{h}} h+\frac{1}{4} h^{2}\right) \frac{1}{4}\left(\overleftarrow{\Gamma}^{\delta}-\vec{\Gamma}^{\delta}\right) \cdot\left(\overleftarrow{\Gamma}_{\delta}-\vec{\Gamma}_{\delta}\right) \\
=\frac{1}{2}\left(\partial^{\delta} \mathrm{h}\right)\left(\partial_{\delta} \mathrm{h}\right)+\underbrace{\left(m_{\mathrm{h}} h+\frac{1}{4} h^{2}\right) \frac{1}{4}\left(\overleftarrow{\Gamma}^{\delta}-\vec{\Gamma}^{\delta}\right) \cdot\left(\overleftarrow{\Gamma}_{\delta}-\vec{\Gamma}_{\delta}\right)}_{\text {Gauge-Higgs interactions terms. }}+\underbrace{m_{\mathrm{h}}^{2} \frac{1}{4}\left(\overleftarrow{\Gamma}^{\delta}-\vec{\Gamma}^{\delta}\right) \cdot\left(\overleftarrow{\Gamma}_{\delta}-\vec{\Gamma}_{\delta}\right)}_{\text {Gauge bosons mass terms }}
\end{gathered}
$$

Hence, to obtain the masses of all gauge bosons, we need to investigate the term:

$$
m_{\mathrm{h}}^{2 \frac{1}{4}}\left(\overleftarrow{\Gamma}^{\delta}-\vec{\Gamma}^{\delta}\right) \cdot\left(\overleftarrow{\Gamma}_{\delta}-\vec{\Gamma}_{\delta}\right)
$$

Or, equivalently:

$$
\pi \alpha m_{\mathrm{h}}^{2}\left(\frac{\overleftarrow{\Gamma}^{\delta}-\vec{\Gamma}^{\delta}}{\sqrt{4 \pi \alpha}}\right) \cdot\left(\frac{\overleftarrow{\Gamma}_{\delta}-\vec{\Gamma}_{\delta}}{\sqrt{4 \pi \alpha}}\right)
$$




\section{Unified Charge Vectors Theory}

To compute (4.6-6), we make use of (4.5-8) and (4.5-9), according to which:

$$
\frac{\overleftarrow{\Gamma}_{n}}{\sqrt{4 \pi \alpha}}=\frac{\mathrm{B}_{n}}{2 \theta_{\mathrm{c}}}\left[\begin{array}{cc}
\mathfrak{T} & 0 \\
0 & \mathfrak{T}
\end{array}\right]+\left[\begin{array}{ll}
\mathbf{G}_{n} & 0 \\
0 & \mathbf{G}_{n}
\end{array}\right]+\frac{\mathbf{W}_{n}}{2 \theta_{\mathbf{s}}}\left[\begin{array}{rr}
\mathbf{I} & 0 \\
0 & -\mathbf{I}
\end{array}\right]+\left[\begin{array}{ll}
\overleftarrow{\widetilde{W}}_{n} & \overleftarrow{\mathbf{W}}_{n}^{+} \\
\overleftarrow{\mathbf{W}}_{n}^{-} & \overleftarrow{\mathbf{W}}_{n}
\end{array}\right]
$$

And (since by (4.5-43) $\left.\overrightarrow{\widetilde{W}}_{n}=0 ; \overrightarrow{\widetilde{W}}_{n}^{ \pm}=0\right)$ :

$$
\frac{\overrightarrow{\boldsymbol{\Gamma}}_{n}}{\sqrt{4 \pi \alpha}}=\frac{\mathrm{B}_{n}}{2 \theta_{\mathrm{c}}}\left[\begin{array}{cc}
\mathfrak{T} & 0 \\
0 & \mathfrak{T}
\end{array}\right]+\left[\begin{array}{ll}
\mathbf{G}_{n} & 0 \\
0 & \mathbf{G}_{n}
\end{array}\right]+\frac{\mathrm{B}_{n}}{2 \theta_{\mathrm{c}}}\left[\begin{array}{rr}
\mathbf{I} & 0 \\
0 & -\mathbf{I}
\end{array}\right]
$$

This implies that:

$$
\frac{\overleftarrow{\Gamma}_{n}-\overrightarrow{\boldsymbol{\Gamma}}_{n}}{\sqrt{4 \pi \alpha}}=\left[\begin{array}{cc}
\left(\frac{1}{2 \theta_{\mathrm{s}}} \mathrm{W}_{n}-\frac{1}{2 \theta_{\mathrm{c}}} \mathrm{B}_{n}\right) \mathbf{I} & 0 \\
0 & -\left(\frac{1}{2 \theta_{\mathrm{s}}} \mathrm{W}_{n}-\frac{1}{2 \theta_{\mathrm{c}}} \mathrm{B}_{n}\right) \mathbf{I}
\end{array}\right]+\left[\begin{array}{cc}
\overleftarrow{\widetilde{\mathbf{W}}}_{n} & \overleftarrow{\widetilde{\mathbf{W}}}_{n}^{+} \\
\overleftarrow{\widetilde{\mathbf{W}}}_{n}^{-} & \overleftarrow{\mathbf{W}}_{n}
\end{array}\right]
$$

We also recall that by (2.3-9) we have $\mathrm{B}_{n}=\theta_{\mathrm{c}} \mathrm{A}_{n}-\theta_{s} \mathrm{Z}_{n}$ and $\mathrm{W}_{n}=\theta_{s} \mathrm{~A}_{n}+\theta_{\mathrm{c}} \mathrm{Z}_{n}$, so:

$$
\frac{1}{2 \theta_{\mathrm{s}}} \mathrm{W}_{n}-\frac{1}{2 \theta_{\mathrm{c}}} \mathrm{B}_{n}=\frac{1}{2}\left(\frac{\theta_{\mathrm{c}}}{\theta_{\mathrm{s}}}+\frac{\theta_{\mathrm{s}}}{\theta_{\mathrm{c}}}\right) \mathrm{Z}_{n}=\frac{\mathrm{Z}_{n}}{2 \theta_{\mathrm{c}} \theta_{\mathrm{s}}}
$$

This allows us to rewrite (4.6-9) as follows:

Where by (4.5-42):

$$
\frac{\overleftarrow{\boldsymbol{\Gamma}}_{n}-\overrightarrow{\boldsymbol{\Gamma}}_{n}}{\sqrt{4 \pi \alpha}}=\left[\begin{array}{cc}
\frac{\mathrm{Z}_{n}}{2 \theta_{\mathrm{c}} \theta_{\mathbf{s}}} \mathbf{I} & 0 \\
0 & -\frac{\mathrm{Z}_{n}}{2 \theta_{\mathrm{c}} \theta_{\mathbf{s}}} \mathbf{I}
\end{array}\right]+\left[\begin{array}{cc}
\overleftarrow{\mathbf{W}}_{n} & \overleftarrow{\mathbf{W}}_{n}^{+} \\
\overleftarrow{\widetilde{\mathbf{W}}}_{n}^{-} & \overleftarrow{\mathbf{W}}_{n}
\end{array}\right]
$$

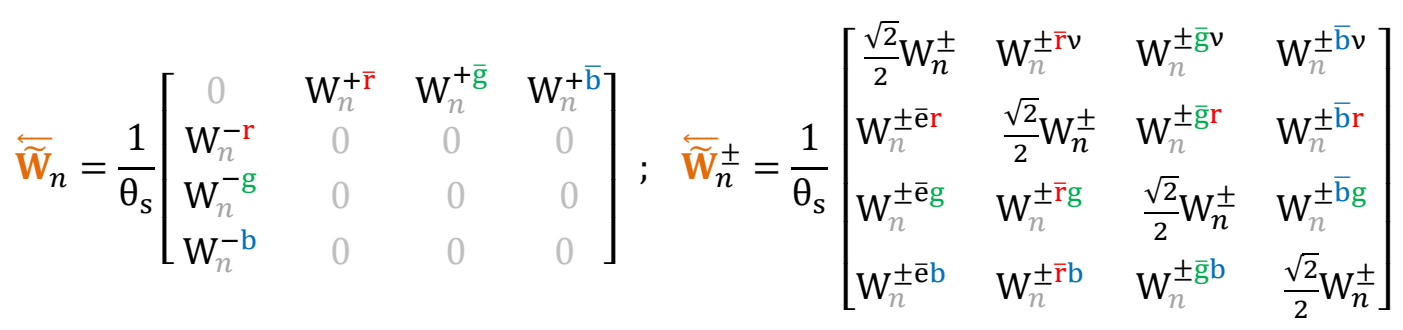

This implies that:

$$
\left(\frac{\overleftarrow{\Gamma}^{\delta}-\vec{\Gamma}^{\delta}}{\sqrt{4 \pi \alpha}}\right) \cdot\left(\frac{\overleftarrow{\Gamma}_{\delta}-\vec{\Gamma}_{\delta}}{\sqrt{4 \pi \alpha}}\right)=\frac{4}{\theta_{\mathrm{s}}^{2}}\left(\begin{array}{c}
\frac{1}{\theta_{\mathrm{c}}^{2}}\left(\mathrm{Z}^{\delta} \mathrm{Z}_{\delta}\right) \\
+\mathrm{W}_{-}^{\delta} \mathrm{W}_{\delta}^{+}+\sum_{c} \mathrm{~W}_{-\overline{\mathrm{c}}}^{\delta} \mathrm{W}_{\delta}^{+\overline{\mathrm{c}}}+\sum_{x, y} \mathrm{~W}_{-x \overline{\mathrm{y}}}^{\delta} \mathrm{W}_{\delta}^{+\overline{\mathrm{x}} y}
\end{array}\right)
$$




\section{Unified Charge Vectors Theory}

Plugging this in (4.6-6), we conclude that:

$$
\pi \alpha m_{\mathrm{h}}^{2}\left(\frac{\overleftarrow{\Gamma}^{\delta}-\vec{\Gamma}^{\delta}}{\sqrt{4 \pi \alpha}}\right) \cdot\left(\frac{\overleftarrow{\Gamma}_{\delta}-\vec{\Gamma}_{\delta}}{\sqrt{4 \pi \alpha}}\right)=\left(\begin{array}{c}
\left(m_{\mathrm{h}} \frac{\sqrt{4 \pi \alpha}}{\theta_{\mathrm{c}} \theta_{\mathrm{s}}}\right)^{2}\left(\mathrm{Z}^{\delta} \mathrm{Z}_{\delta}\right) \\
+\left(m_{\mathrm{h}} \frac{\sqrt{4 \pi \alpha}}{\theta_{\mathrm{s}}}\right)^{2}\left(\mathrm{~W}_{-}^{\delta} \mathrm{W}_{\delta}^{+}+\sum_{c} \mathrm{~W}_{-\overline{\mathrm{c}}}^{\delta} \mathrm{W}_{\delta}^{+\overline{\mathrm{c}}}+\sum_{x, y} \mathrm{~W}_{-x \overline{\mathrm{y}}}^{\delta} \mathrm{W}_{\delta}^{+\overline{\mathrm{x}} y}\right)
\end{array}\right)
$$

Our first observation regards the absence of the electromagnetic and gluon fields in (4.6-14). This implies that the photon and gluons are massless, as expected. Notice that this is a prediction of the model, rather than a presupposition.

Our next prediction is the relation between the masses of the $\mathrm{Z}$ boson and the Higgs:

$$
m_{\mathrm{z}}=m_{\mathrm{h}} \frac{\sqrt{4 \pi \alpha}}{\theta_{\mathrm{c}} \theta_{\mathrm{s}}} \text { and hence } \frac{1}{\alpha}=\frac{4 \pi}{\theta_{\mathrm{c}}^{2} \theta_{\mathrm{s}}^{2}} \frac{m_{\mathrm{h}}^{2}}{m_{\mathrm{z}}^{2}}
$$

Let us plug in the numbers and see if we get a reasonable value for the fine structure coefficient $\alpha$. By (4.5-34) we have:

$$
\theta_{\mathrm{s}}^{2}=\frac{3}{13} \text { and hence } \theta_{\mathrm{c}}^{2}=1-\theta_{\mathrm{s}}^{2}=\frac{10}{13}
$$

We also know from measurements that:

$$
\frac{m_{\mathrm{h}}^{2}}{m_{\mathrm{z}}^{2}} \approx \frac{(125.35)^{2}}{(91.18)^{2}}
$$

Plugging these numbers into (4.6-15), we conclude that:

$$
\frac{1}{\alpha} \approx 4 \pi \frac{13}{3} \frac{13}{10} \frac{(125.35)^{2}}{(91.18)^{2}} \approx 133.8
$$

This is a sensible result, considering the fact that the measured value of $\frac{1}{\alpha}$ runs between 127 and 137 depending on the energy used. It is also worth mentioning that the unified Lagrangian is not making use of a so-called "Higgs vacuum expectation energy", which is usually denoted by $v$ and is given the value $\sqrt{2} \cdot 180 \mathrm{GeV}$. Realizing that $2 m_{\mathrm{h}} \approx v(1-0.015)$, we speculate that $v \stackrel{\text { def }}{=} 2 m_{\mathrm{h}}$. This allows us to eliminate $v$ from the unified Lagrangian. However, if this speculation turns out to be wrong, one can always introduce $v$ back into the Lagrangian and thus obtain full consistency with the traditional form of the standard model Lagrangian.

Finally, we conclude that the mass of all the charged bosons $\mathrm{W}_{n}^{ \pm}, \mathrm{W}_{n}^{ \pm \mathrm{c}}$, and $\mathrm{W}_{n}^{ \pm \overline{\mathrm{x}} \mathrm{y}}$ is the same, and is equal to:

$$
m_{\mathrm{w}}^{ \pm}=m_{\mathrm{h}} \frac{\sqrt{4 \pi \alpha}}{\theta_{\mathrm{s}}} \approx 125.35 \sqrt{\frac{13}{3} \frac{4 \pi}{133.8}} \approx 80[\mathrm{GeV}]
$$




\section{Unified Charge Vectors Theory}

\subsection{The Higgs Boson}

In this section, we consider the Higgs scalar field resulting from the following Lagrangian terms:

$$
\left(\square^{\delta} \mathbf{H}\right)^{\dagger} \cdot\left(\square_{\delta} \mathbf{H}\right)-\left(\mathbf{H}^{\dagger} \mathbf{H}-\frac{1}{4} m_{\mathrm{h}}^{2} \mathbb{I}\right) \cdot\left(\mathbf{H}^{\dagger} \mathbf{H}-\frac{1}{4} m_{\mathrm{h}}^{2} \mathbb{I}\right)-\overleftarrow{\bar{\Psi}} \cdot \mathbf{M} \vec{\Psi}-\overrightarrow{\bar{\Psi}} \cdot \mathbf{M} \overleftarrow{\Psi}
$$

Working in the Higgs diagonal frame, we use (4.3-9), (4.3-10), (4.4-8), and (4.6-3) to write:

$$
\mathbf{H} \stackrel{\text { def }}{=} \frac{1}{2} m_{\mathrm{h}} \boldsymbol{\Phi}=\frac{1}{2} m_{\mathrm{h}} e^{\phi} \mathbb{I}=\frac{1}{2}\left(m_{\mathrm{h}}+\frac{1}{2} \mathrm{~h}\right) \mathbb{I}
$$

This implies that:

And:

$$
\mathbf{M}=\boldsymbol{m} \backslash \mathbb{I} e^{\phi}=\boldsymbol{m} \backslash \mathbb{I}\left(1+\frac{\mathrm{h}}{2 m_{\mathrm{h}}}\right)
$$

$$
\mathbf{H}^{\dagger} \mathbf{H}=\frac{1}{4}\left(m_{\mathrm{h}}+\frac{1}{2} \mathrm{~h}\right)^{2} \mathbb{I}=\frac{1}{4}\left(m_{\mathrm{h}}^{2}+m_{\mathrm{h}} \mathrm{h}+\frac{1}{4} \mathrm{~h}^{2}\right) \mathbb{I}
$$

Using the fact that $\mathbb{I} \cdot \mathbb{I}=8$, we see that:

$$
\begin{gathered}
\left(\mathbf{H}^{\dagger} \mathbf{H}-\frac{1}{4} m_{\mathrm{h}}^{2} \mathbb{I}\right) \cdot\left(\mathbf{H}^{\dagger} \mathbf{H}-\frac{1}{4} m_{\mathrm{h}}^{2} \mathbb{I}\right)=\left(m_{\mathrm{h}} \mathrm{h}+\frac{1}{4} \mathrm{~h}^{2}\right)\left(\frac{1}{4} \mathbb{I} \cdot \frac{1}{4} \mathbb{I}\right)\left(m_{\mathrm{h}} \mathrm{h}+\frac{1}{4} \mathrm{~h}^{2}\right) \\
=\frac{1}{2}\left(m_{\mathrm{h}} \mathrm{h}+\frac{1}{4} \mathrm{~h}^{2}\right)^{2}=\frac{1}{2} m_{\mathrm{h}}^{2} \mathrm{~h}^{2}+\frac{1}{4} m_{\mathrm{h}} \mathrm{h}^{3}+\frac{1}{32} \mathrm{~h}^{4}
\end{gathered}
$$

We also recall that, by (4.6-4), we have:

$$
\left(\square^{\delta} \mathbf{H}\right)^{\dagger} \cdot\left(\square_{\delta} \mathbf{H}\right)=\frac{1}{2}\left(\partial^{\delta} \mathrm{h}\right)\left(\partial_{\delta} \mathrm{h}\right)+\left(\begin{array}{c}
+m_{\mathrm{h}}^{2} \frac{1}{4}\left(\overleftarrow{\Gamma}^{\delta}-\vec{\Gamma}^{\delta}\right) \cdot\left(\overleftarrow{\Gamma}_{\delta}-\vec{\Gamma}_{\delta}\right) \\
+\left(m_{\mathrm{h}} h+\frac{1}{4} h^{2}\right) \frac{1}{4}\left(\overleftarrow{\Gamma}^{\delta}-\vec{\Gamma}^{\delta}\right) \cdot\left(\overleftarrow{\Gamma}_{\delta}-\vec{\Gamma}_{\delta}\right)
\end{array}\right)
$$

Combining all these results, we can rewrite (4.7-1) as follows:

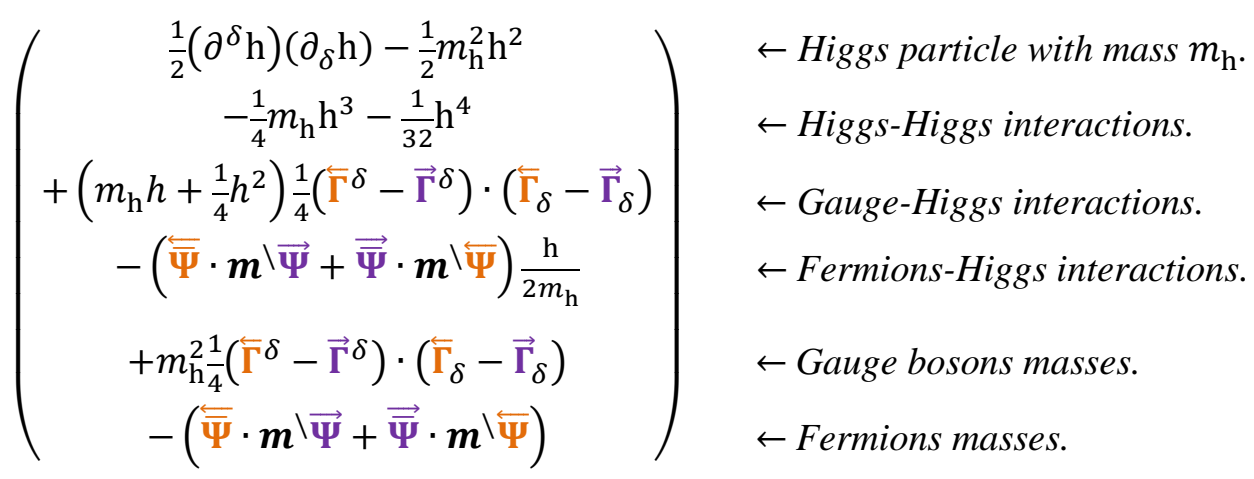




\section{Unified Charge Vectors Theory}

It is worth reiterating that the unified Lagrangian is not making use of a so-called "Higgs vacuum expectation energy", which is usually denoted by $v$ and is given the value $\sqrt{2} \cdot 180 \mathrm{GeV}$. Realizing that $2 m_{\mathrm{h}} \approx v(1-0.015)$, we speculate that $v \stackrel{\text { def }}{=} 2 m_{\mathrm{h}}$. This allows us to eliminate $v$ from the unified Lagrangian. However, if this speculation turns out to be wrong, one can always introduce $v$ back into the Lagrangian and thus obtain full consistency with the traditional form of the standard model Lagrangian. 


\section{Unified Charge Vectors Theory}

\section{Summary}

We have managed to derive the standard model (and more) from a simple unified charge vectors quantization equation.

Using the unified charge vectors conservation law, we have deduced the bosons periodic table.

We then integrated all these results into a unified Lagrangian. Requesting invariance under left and right $U(8)$ transformations, we have deduced the existence of the Higgs matrix field, which in turn, allowed us to predict the masses of all the gauge bosons.

\section{References}

1. An Introduction to the Standard Model of Particle Physics, Second Edition

By W. N. COTTINGHAM and D. A. GREENWOOD, University of Bristol, UK

2. Introduction to Elementary Particles, Second edition, by David Griffiths.

3. Wikipedia. 